\title{
IFITM3 functions as a PIP3 scaffold to amplify PI3K signalling in B cells
}

https://doi.org/10.1038/s41586-020-2884-6

Received: 11 September 2019

Accepted: 13 August 2020

Published online: 4 November 2020

Check for updates

\author{
Jaewoong Lee', Mark E. Robinson', Ning Ma², Dewan Artadji', Mohamed A. Ahmed ${ }^{3}$, \\ Gang Xiao ${ }^{3}$, Teresa Sadras', Gauri Deb ${ }^{3}$, Janet Winchester ${ }^{3}$, Kadriye Nehir Cosgun', \\ Huimin Geng', Lai N. Chan', Kohei Kume', Teemu P. Miettinen ${ }^{5,6}$, Ye Zhang ${ }^{5}$, Matthew A. Nix ${ }^{4}$, \\ Lars Klemm ${ }^{1}$, Chun Wei Chen ${ }^{3}$, Jianjun Chen $^{3}$, Vishal Khairnar ${ }^{3}$, Arun P. Wiita ${ }^{4}$, \\ Andrei Thomas-Tikhonenko ${ }^{7}$, Michael Farzan ${ }^{8}$, Jae U. Jung ${ }^{9}$, David M. Weinstock ${ }^{10,11}$, \\ Scott R. Manalis ${ }^{5,12}$, Michael S. Diamond ${ }^{13,14,15}$, Nagarajan Vaidehi ${ }^{2}$ \&arkus Müschen ${ }^{1,16} \bowtie$
}

Interferon-induced transmembrane protein 3 (IFITM3) has previously been identified as an endosomal protein that blocks viral infection ${ }^{1-3}$. Here we studied clinical cohorts of patients with B cell leukaemia and lymphoma, and identified IFITM 3 as a strong predictor of poor outcome. In normal resting B cells, IFITM3 was minimally expressed and mainly localized in endosomes. However, engagement of the $B$ cell receptor (BCR) induced both expression of IFITM3 and phosphorylation of this protein at Tyr20, which resulted in the accumulation of IFITM3 at the cell surface. In B cell leukaemia, oncogenic kinases phosphorylate IFITM3 at Tyr20, which causes constitutive localization of this protein at the plasma membrane. In a mouse model, Ifitm $3^{-/-}$naive B cells developed in normal numbers; however, the formation of germinal centres and the production of antigen-specific antibodies were compromised. Oncogenes that induce the development of leukaemia and lymphoma did not transform Ifitm3 ${ }^{-/-}$B cells. Conversely, the phosphomimetic IFITM3(Y2OE) mutant induced oncogenic PI3K signalling and initiated the transformation of premalignant $B$ cells. Mechanistic experiments revealed that IFITM3 functions as a PIP3 scaffold and central amplifier of PI3K signalling. The amplification of PI3K signals depends on IFITM3 using two lysine residues (Lys83 and Lys104) in its conserved intracellular loop as a scaffold for the accumulation of PIP3. In Ifitm $3^{-/-}$B cells, lipid rafts were depleted of PIP3, which resulted in the defective expression of over 60 lipid-raft-associated surface receptors, and impaired BCR signalling and cellular adhesion. We conclude that the phosphorylation of IFITM 3 that occurs after $B$ cells encounter antigen induces a dynamic switch from antiviral effector functions in endosomes to a PI3K amplification loop at the cell surface. IFITM3-dependent amplification of PI3K signalling, which in part acts downstream of the BCR, is critical for the rapid expansion of $B$ cells with high affinity to antigen. In addition, multiple oncogenes depend on IFITM 3 to assemble PIP3-dependent signalling complexes and amplify PI3K signalling for malignant transformation.
IFITM3 is a small antiviral membrane protein (133 amino acids in size) with activity against influenza A virus, severe acute respiratory syndrome (SARS) coronavirus and many other viruses ${ }^{1-5}$. Endocytosis and preferential endosomal localization of IFITM 3 are mediated by its $\mathrm{N}$-terminal AP2 sorting motif, YEML ${ }^{1}$. Phosphorylation of this motif at $\mathrm{Y} 20$ by SRC kinases prevents not only endocytosis but also proteasomal degradation ${ }^{6,7}$. Individuals with the IFITM3 single-nucleotide polymorphism (SNP) rs12252-C allele show increased morbidity and mortality from H1N1 strains of influenza $A$ virus ${ }^{8}$ and SARS coronavirus $2^{4}$. IFITM3SNP rs12252-C was predicted through mRNA splicing to encode a 21-amino-acid truncation of the $\mathrm{N}$ terminus (which includes Y20), although a recent transcript analysis found no evidence for such processed RRNA $^{9}$. IFITM3 probably functions as an antiviral effector molecule by limiting viral fusion through increasing the 'rigidity' of endosomal cell membranes ${ }^{10,11}$. IFITM3 is overexpressed in multiple types of cancer, and its expression levels correlated with histopathological grading and staging ${ }^{12,13}$. Although IFITM3-dependent antiviral effector functions are restricted to endosomal compartments ${ }^{14}$, we report here on IFITM3-dependent functions at the cell surface of B cells, where IFITM3 functions as amplifier of PI3K signalling. 


\section{IFITM3 is essential for $B$ cell affinity maturation}

Although IFITM3 is only minimally expressed in normal resting B cells, BCR engagement and acute activation of the PI3K pathway strongly induced the expression of this protein (Extended Data Fig. 1a-c). Expression of IFITM proteins has recently been established as a diagnostic biomarker of high-risk B cell-lineage acute lymphoblastic leukaemia (B-ALL) that expresses the oncogenic BCR-ABL1 tyrosine kinase or related kinase ${ }^{15}$; we found that BCR-ABL1 kinase inhibition decreased levels of IFITM3 expression (Extended Data Fig. 1d). Levels of IFITM3 mRNA were generally higher in B cell malignancies than in their normal counterparts (Extended Data Fig. 1f, g) and elevated IFITM3 mRNA levels were associated with poor clinical outcomes in multiple clinical cohorts of patients with B cell malignancies (including paediatric and adult B-ALL and mantle cell lymphoma) (Extended Data Fig. 1h-l). IFITM3 was previously identified as a transcriptional target of the transcriptional repressor IKZF ${ }^{16}$, which suggests that high levels of IFITM3 expression may be the result of genetic lesions in $I K Z F 1$. Our results confirmed that reconstitution of IKZF1 suppresses IFITM3 expression, and that inducible deletion of $I k z f 1$ in mice relieves transcriptional repression (Extended Data Fig. $1 \mathrm{~m}, \mathrm{n}$ ). However, a multivariate analysis of risk factors in B-ALL showed that high IFITM3 mRNA levels are an independent predictor of poor outcome, regardless of $I K Z F 1$ deletion (Extended Data Fig. 10). We next examined how the genetic deletion of Ifitm 3 affects normal B cell development in mice. Surface expression of CD19-a common B cell surface receptor that promotes SRC kinase and PI3K signalling ${ }^{17}$ - was substantially diminished in Ifitm $3^{-/-}$ pre-B cells (Fig. 1a). Consistent with increased apoptosis and reduced proliferation, expression of MYC and BCL2 as well as PI3K signalling were reduced in 1 fitm $3^{-/-}$pre-B cells (Fig. 1a-c). The development of resting Ifitm $3^{-/}$B cell populations was largely unperturbed (Extended Data Fig. 2), whereas B1 cell and marginal-zone B cell populations were decreased (Fig. 1d, Extended Data Fig. 2). To assess B-cell-specific effects of IFITM3 during humoral immune responses, we performed adoptive transfer experiments: Ifitm $3^{+/+}$or Ifitm $3^{-/-}$splenic B cells were transferred into congenic $\mu \mathrm{MT}$ mice that lack endogenous mature B cells. After reconstitution of mature B cell development, recipient $\mu$ MT mice were immunized with the hapten 4-hydroxy-3-nitrophenyl acetyl (NP) conjugated to keyhole limpet haemocyanin (KLH) (NP-KLH). Upon immunization, $\mu \mathrm{MT}$ mice reconstituted with $/$ fitm $^{+/+} \mathrm{B}$ cells developed about $5 \%$ NP-specific germinal-centre B cells, which were largely absent in $\mu \mathrm{MT}$ mice reconstituted with Ifitm $^{-/-}$B cells (Fig. 1e-h). Similarly, the number of follicles in the spleen, $\mathrm{PNA}^{+}$germinal-centre $\mathrm{B}$ cells and production of IgM, IgG1 and IgG2b antibodies were reduced in the absence of Ifitm3 (Fig. 1i). These abnormalities in Ifitm3-deficient B cells phenocopy B cell defects seen in mice upon deletion of $P i k 3 c d^{18}$ and $C d 19^{19}$.

\section{Role of Ifitm3 in oncogenic signalling}

To elucidate a potential contribution of Ifitm 3 to oncogenic signalling, we studied the transformation of $I$ fitm $3^{+/+}$and $I$ fitm $3^{-/-}$pre-B cells by $B C R-A B L 1$ and $N R A S^{G 12 D}$ (Fig. 2a, b). As in normal B cells, Ifitm $3^{-/-}$leukaemia cells in a mouse model of B-ALL (Ifitm ${ }^{-/-}$B-ALL cells) showed multiple defects in survival and proliferation, and a markedly reduced ability to form colonies in semi-solid methylcellulose (Fig. 2b, c). Consistent with a 70 -fold reduction of the frequency of leukaemia-initiating cells $\left(P=3.7 \times 10^{-7}\right)$, Ifitm $3^{-/-}$B-ALL cells did not initiate overt leukaemia in immunodeficient transplant recipient mice (Fig. 2d, e). Similar to B-ALL cells, deletion of IFITM3 in mantle cell lymphoma (MCL) cells reduced competitive fitness and proliferation (Fig. 2f). Both mouse fitm $^{-1-}$ $\mathrm{B}$ cells and human IFITM3-deficient MCL cells showed defective $\mathrm{Ca}^{2+}$ release in response to $B C R$ engagement, and partially lost CD19 surface expression (Extended Data Fig. 2c, d). In addition, BCR engagement in IFITM3 $^{-/-} \mathrm{MCL}$ cells did not induce processive signal amplification between $\mathrm{CD} 19$ and $\mathrm{LYN}^{17}$, as CD19 was not phosphorylated and did not interact with LYN. IFITM3-deficient MCL cells expressed lower levels of LYN and did not activate SRC kinases in response to BCR engagement (Extended Data Fig. 2e). RNA-sequencing gene-expression studies revealed upregulation of Ifitm 1 in $I$ fitm $3^{-/-}$B-ALL cells, presumably to compensate for loss of Ifitm3. Gene set enrichment analyses showed that Ifitm 3 deficiency was associated with reduced expression of components of the BCR signalling pathway and a phenotype reminiscent of B cell anergy (Extended Data Fig. 3a-c).

\section{Effect of phosphorylation of IFITM3 at Y20}

Combinations of surface and intracellular staining revealed the cytoplasmic localization of the N terminus of IFITM3, with a short extracellular portion of the $\mathrm{C}$ terminus (Extended Data Fig. 3d). Previous studies have shown that phosphorylation of IFITM3 at Y20 prevents both endocytosis and degradation ${ }^{6,7}$. Phosphomimetic IFITM3(Y20E) was constitutively localized at the cell surface. Similarly, phosphorylation by BCR-ABL1 induced the accumulation of IFITM3 at the cell membrane, in a process that was sensitive to inhibition of BCR-ABL1 or SRC kinases (Extended Data Fig. 3e). Therefore, the phosphorylation of $\mathrm{Y} 20$ induces a dynamic switch from antiviral functions in endosomes to BCR or oncogene signalling at the cell surface (Extended Data Fig. 3e,f). To test whether phosphorylation of IFITM3 at Y20 and constitutive membrane localization are sufficient to enable oncogenic signalling, we overexpressed wild-type and IFITM3(Y2OE) in premalignant $\mathrm{B}$ cells carrying a $B C R-A B L 1$-knock-in allele, which did not readily give rise to leukaemia ${ }^{20}$. Expression of IFITM3(Y20E) increased the ability of pre-B cells to form colonies by 129 -fold $\left(P=2.1 \times 10^{-8}\right)$ (Fig. 2g), induced oncogenic signalling and initiated fatal leukaemia in congenic transplant recipient mice (Fig. $2 \mathrm{~h}-\mathrm{j}$ ). To elucidate how membrane-bound IFITM3(Y2OE) promotes malignant transformation, we performed phosphoproteomic analyses of IFITM3 Y20 signalling in patient-derived B-ALL cells. Consistent with processive signal amplification $^{17}$, engagement of IFITM3 induced phosphorylation of CD19 on multiple residues-including Y531, which mediates PI3K activation. In addition, Y20 of IFITM3 engaged multiple components of the BCR and integrin receptor signalling pathways (Fig. 3a, Extended Data Fig. 4a), which converge with CD19 at the level of PI3K activation. A comprehensive analysis of phosphosites showed a significant enrichment of phosphorylation in the PI3K, BCR and integrin receptor pathways, which we confirmed for SRC, CD19 and PI3K signalling by western blot (Fig. 3a-c). IFITM3(Y2OE) required crosslinking by a full antibody, as the engagement of $Y 20$ of monomeric IFITM3 using $F(a b)$ fragments did not elicit PI3K activation (Extended Data Fig. 4b). Because PI3K signalling in mouse B-ALL cells increased Ifitm $3 \mathrm{mRNA}$ levels by 10 -fold and protein levels by more than 50 -fold, these observations suggest a feed-forward loop of signal amplification between IFITM3 and PI3K signalling (Extended Data Fig. 1b-e). Comparable to effects on BCR signalling and CD19 surface expression in B cells, genetic deletion of IFITM3 in human Jurkat T cells compromised T cell receptor signalling and CD3 surface expression (Extended Data Fig. 4c), which suggests that IFITM3 may have a similar role in T cells.

\section{IFITM3 enables CD19-PI3K signalling}

By enriching for cell-surface proteins using $\mathrm{N}$-linked glycosylation-site biotin labelling, we identified 65 surface receptors-most of which were associated with lipid rafts - that were downregulated in Ifitm $3^{-/-}$B-ALL cells, including CD19, BCR-associated and adhesion receptors. Only five surface proteins were upregulated-including the IFITM family member IFITM2, which suggests a possible compensation mechanism (Extended Data Fig. 4d). We performed validation by flow cytometry for 17 surface receptors for IFITM3 loss-of-function $\left(\right.$ Ifitm $^{-/-}$) and gain-of-function (IFITM3(Y2OE)) (Extended Data Fig. 4e-g). Continuous 

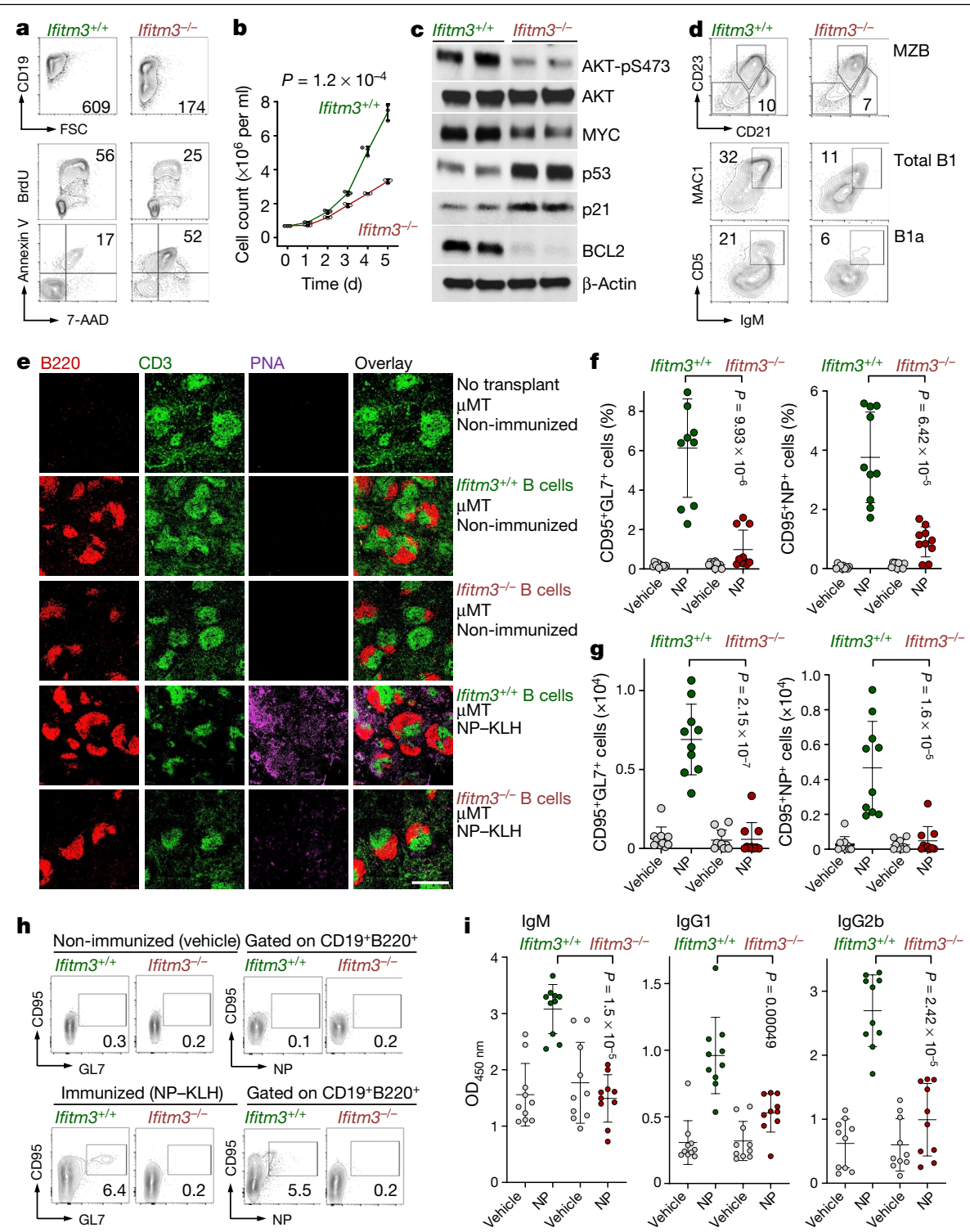

Fig. $1 \mid$ Ifitm 3 is essential for $B$ cell activation and affinity maturation in germinal centres. a, CD19 surface expression (top), cell cycle progression (middle) (percentages in S phase) and cell viability (bottom) (percentages of annexin $\mathrm{V}^{+} 7-\mathrm{AAD}^{+}$cells) were measured. b, Number of viable Ifitm $^{+/+}$and Ifitm $3^{-/-}$pre-B cells were counted at the times indicated. c, Levels of AKT phosphorylated at S473 (AKT-pS473), AKT, MYC, p53, p21 and BCL2 were assessed in Ifitm $3^{+/+}$and $I$ fitm $3^{-/-}$pre-B cells. d, Splenic B cells from $/$fitm $^{+/+}$and Ifitm $3^{-/-}$mice $(n=5)$ were analysed for $\mathrm{CD} 21^{\text {high }} \mathrm{CD} 23^{\text {low }}$ or $\mathrm{CD} 21^{\text {high }} \mathrm{CD} 23^{-}$ marginal-zone $B$ cells (MZB), peritoneal-cavity B cells for $\mathrm{MAC1}^{+}$IgM $^{+} \mathrm{B} 1$ cells and $\mathrm{CD}^{+}{ }^{+} \mathrm{gM}^{+}$B1a cells. e, Splenic B cells from $I$ fitm $^{+/ /}$and $/$fitm $^{-/-}$mice were adoptively transferred to $\mu \mathrm{MT}$ recipient mice $(n=10)$ followed by immunization with $0.5 \mathrm{mg}$ of NP-KLH or vehicle. Spleens were collected on

forced expression of CD19 (for more than one week) in 1 fitm $^{-/-}$B cells partially restored defective survival and proliferation, which required the PI3K-activation motif Y531 of CD19 (ref. ${ }^{21}$ ) (Extended Data Fig. 5a-e). However, inducible translocation of CD19 to the cell membrane did not restore any defects in Ifitm $3^{-/-}$B cells, which suggests that IFITM3 is still required to integrate CD19 into signalling complexes for SRC kinase and PI3K signalling (Extended Data Fig. 5f-h). To study complexes that interact with IFITM3, we performed enzyme-catalysed proximity labelling studies on the basis of $\mathrm{N}$-terminal fusions of the BirA biotin ligase with IFITM3(Y2OE) carrying an extracellular haemagglutinin (HA) tag in B-ALL and IgM $^{+}$MCL cells. After engagement with IFITM3 (anti-HA) or BCR (anti-IgM), IFITM3-interacting proteins

day 12 after immunization and subjected to immunofluorescence staining of tissue sections with B220, CD3 and peanut agglutinin (PNA). Scale bar, $500 \mu \mathrm{m}$. f-h, Splenocytes collected from $\mu \mathrm{MT}$ mice $(n=10)$ were analysed by flow cytometry 12 days after immunization for CD95, GL7 and NP to identify NP-specific germinal-centre B cells. Relative fractions (f), absolute numbers (g) and representative flow cytometry plots $(\mathbf{h})$ are shown. i, Levels of serum immunoglobulin isotypes in $\mu \mathrm{MT}$ recipient mice transplanted with Ifitm $^{+/+}$or If itm $3^{-/-}$B cells are shown before and after immunization ( $n=10$; day 12). Serum levels of IgM, IgG1 and IgG2b were determined by enzyme-linked immunosorbent assay (ELISA). For gel source data for a-c, see Supplementary Fig. 1. In b, f, g, i, mean \pm s.d. indicated; significance determined by two-tailed $t$-test.

were biotinylated on the basis of their proximity to the cytoplasmic BirA moiety, collected using streptavidin-coated beads and identified by mass spectrometry. Consistent with phosphoproteomic analyses (Fig. 3a), and across different cell types (B-ALL and MCL) and stimulations (anti-HA and anti-IgM), IFITM3-interacting proteins included BCR, integrin receptor and PI3K signalling elements (Fig. 3d, e, Extended Data Fig. 6a-d). We transduced MCL cells with Flag-IFITM3 for validation by co-immunoprecipitation and western blotting (Fig. 3f, g). Using proximity ligation assays, we confirmed the inducible formation of a complex between IFITM 3 and the BCR signalling chain CD79B upon engagement of the BCR. Interactions between BCR and IFITM3 were induced within minutes and dissociated after $30 \mathrm{~min}$ 


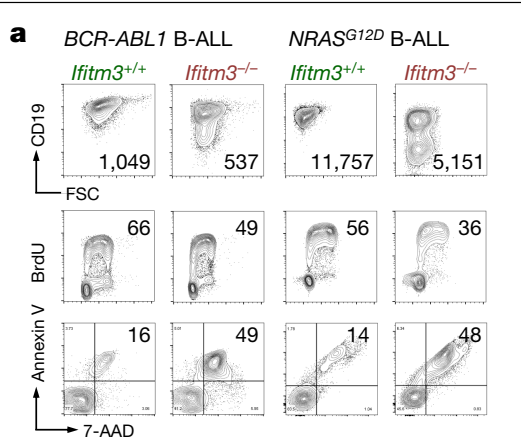

c $B C R-A B L 1 \mathrm{~B}-\mathrm{ALL}$

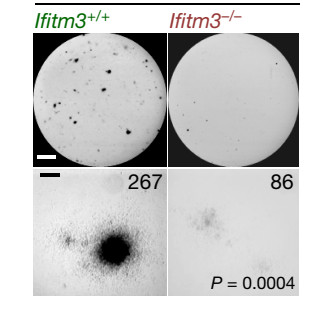

d 100

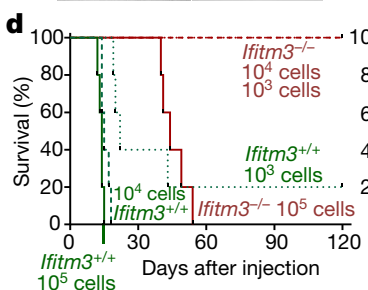

$10^{5}$ cells
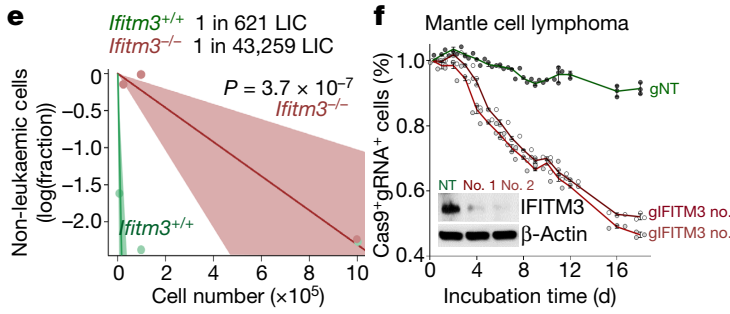

b

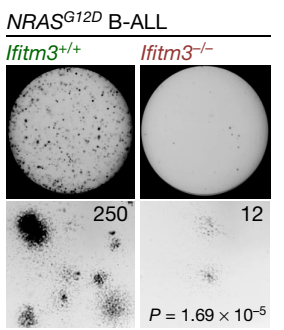

.

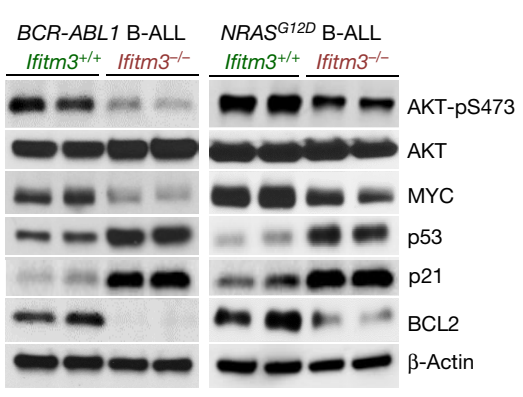

g $L S L-B C r^{B C R-A B L 1} \times M b 1-c r e$
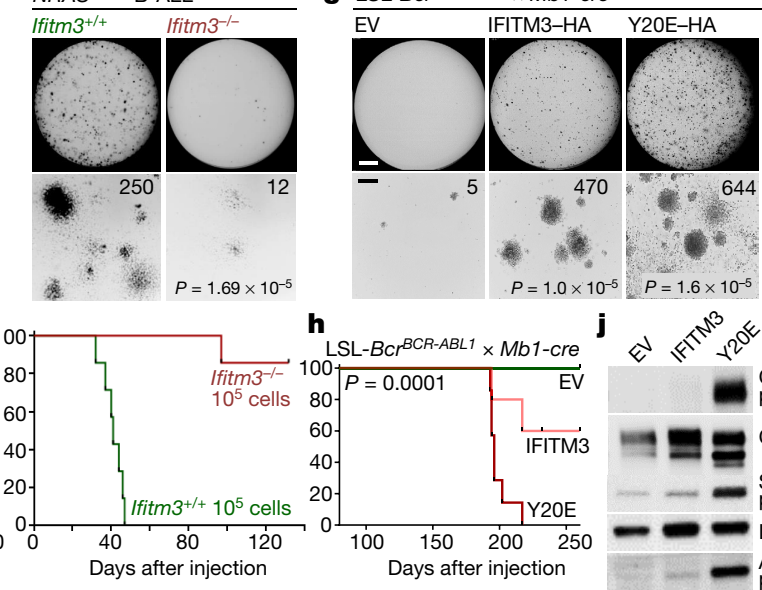

$\Delta \leqslant \pi^{3}+2^{2}$
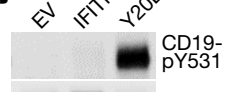

볼 $\mathrm{CD} 19$

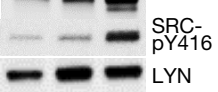

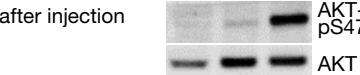

i

LSL-BCr ${ }^{B C R-A B L 1} \times M b 1-c r e$

EV IFITM3-HA Y20E-HA Day

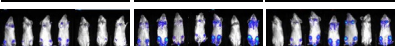

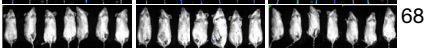

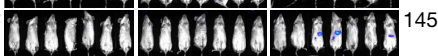

Fig. 2 | Essential role of IFITM3 in oncogenic signalling and $B$ cell transformation.a, CD19 surface expression (top), cell cycle progression (middle) (percentages in S phase) and cell viability (bottom) (percentages of annexin $\mathrm{V}^{+}$7-AAD ${ }^{+}$cells) were measured. b, c, Ifitm $3^{+/+}$and Ifitm $3^{-/-}$B-ALL cells transformed with human $B C R-A B L 1$ or $N R A S^{G 12 D}$ were assayed for levels of AKT-pS473, AKT, MYC, p53, p21 and BCL2 $(n=3)(\mathbf{b})$ and plated in semi-solid methylcellulose (c). Scale bars, $7 \mathrm{~mm}$ (c, top row), $2.5 \mathrm{~mm}$ (c, bottom row). d, Kaplan-Meier analyses of NOD-SCID-gamma (NSG) recipient mice injected with the indicated numbers of $I$ fitm $3^{+/+}$and Ifitm $3^{-/-} B C R-A B L 1$ (left) or $N R A S^{G 12 D}$ (right) B-ALL cells $(n=5)$. e, Frequencies of leukaemia-initiating cells (LIC) estimated with extreme limiting dilution analysis (ELDA) $(90 \%$ confidence interval; likelihood ratio test).f,Jeko1 MCL cells expressing doxycycline (Dox)-inducible Cas9 were transduced with IFITM3-targeting or non-targeting (NT) single-guide RNAs. Enrichment or depletion of targeted cells $\left(\mathrm{Cas}^{+} \mathrm{gRNA}^{+}\right)$was monitored by flow cytometry upon treatment with

(Fig. 3h, i). Consistent with endosomal BCR functions ${ }^{22}$, interactions between BCR and IFITM 3 occasionally colocalized with LAMP1 ${ }^{+}$endosomal compartments (Extended Data Fig. 6e). Therefore, BCR-mediated activation of SRC-mediated phosphorylation of Y20 of IFITM3 induces the accumulation of IFITM3 at the plasma membrane, where IFITM3 functions as a scaffold for CD19 and LYN in proximity to BCR molecules to enable processive signal amplification (Fig. $3 \mathrm{j}$ ). In resting B cells, IgM BCRs are excluded from lipid rafts and recruited upon encounters with antigen ${ }^{23}$. By contrast, IgD BCRs are already in close proximity to CD19 within lipid rafts in resting $B$ cells, which suggests that IFITM3 may specifically enable IgM BCRs. doxycycline (mean \pm s.d.), and IFITM3 levels were measured by western blot. g, Premalignant LSL- $B c r^{B C R-A B L 1} \times M b 1$-cre pre-B cells expressing IFITM3, IFITM3(Y2OE) or empty vector (EV) were plated for colony-forming assays (7 days). Representative images shown at $1 \times($ top $)$ and $10 \times($ bottom) magnification. $\mathbf{h}$, Survival analyses $(P=0.0001$, log-rank test) of congenic recipient mice transplanted with LSL-Bcr ${ }^{B C R-A B L 1} \times M b 1$ - $c r e$ B cells transduced with empty vector, IFITM3 or IFITM3(Y2OE) $(n=7)$. i, The engraftment and expansion of luciferase-labelled leukaemia cells were monitored by luciferase bioimaging at the times indicated.j, Effects of IFITM3 or IFITM3(Y2OE) on oncogenic signalling in $\mathrm{LSL}-B c r^{B C R-A B L 1} \times M b 1$-cre $\mathrm{B}$ cell precursors measured by western blot and compared to empty vector. Phosphorylation of CD19 at Y531 (CD19-pY531), of SRC at Y416 (SRC-pY416) and AKT at S473 were examined. Gel source data for $\mathbf{a}-\mathbf{c}, \mathbf{f}, \mathbf{g}, \mathbf{j}$ are shown in Supplementary Fig. 1. In c, $\mathbf{g}$, two-tailed $t$-test.

\section{IFITM3-PIP3 binding is needed to form lipid rafts}

Reduced expression of integrins and adhesion receptors was associated with decreased homotypic adhesion of $I$ fitm $3^{-/-}$pre-B cells, whereas membrane-bound IFITM3(Y2OE) triggered the formation of large clusters (Extended Data Fig. 7). Because IKZF1 negatively regulates adhesion ${ }^{24,25}$, we examined whether this regulation is mediated by transcriptional repression of Ifitm $3^{16}$. Overexpression of a dominant-negative IKZF1-mutant (termed IK6) markedly increased colony formation, adhesion to stroma and surface expression of adhesion receptors in the presence-but not in the absence-of IFITM3. Although 

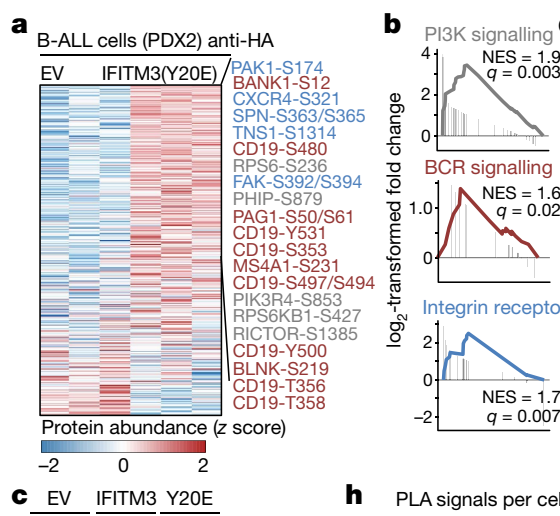

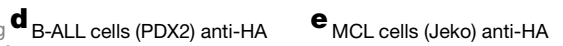

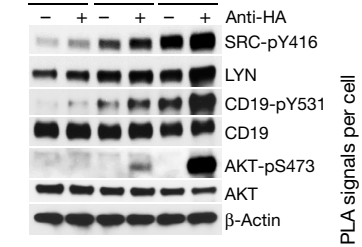

h PLA signals per cell $n=250 ; 380 ; 405 ; 85$
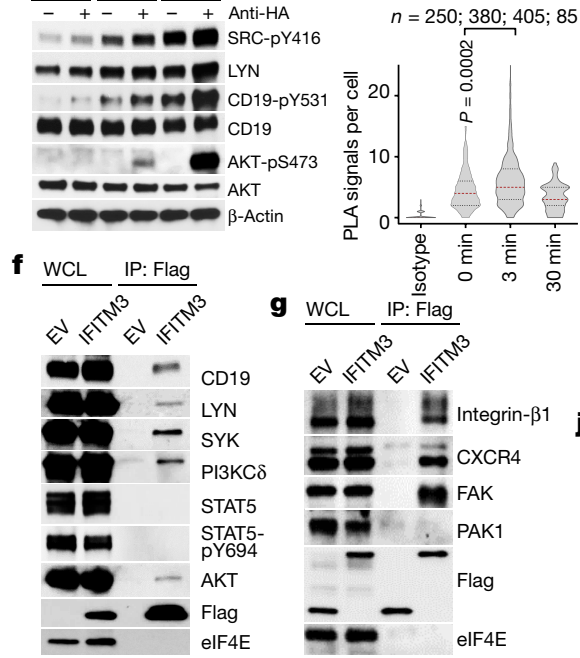

Fig. 3 | IFITM3 links components of the BCR and integrin receptor pathways to PI3K signalling. a, Levels of proteins phosphorylated at the indicated residues in PDX2 B-ALL cells transduced with HA-tagged IFITM3(Y2OE) or empty vector (EV) control were identified by mass spectrometry upon IFITM3 crosslinking with anti-HA antibodies. b, Feature set enrichment analysis (FSEA) for phosphorylated proteins in the BCR (red), integrin (blue) and PI3K (grey) pathways, ranked by $\log _{2}$-transformed fold change (Kolmogorov-Smirnov). c, Levels of SRC-pY416, LYN, CD19-pY531, CD19, AKT-pS473 and AKT assessed in ICN12 cells expressing IFITM3-HA, IFITM3(Y2OE)-HA or HA-tagged empty vector upon IFITM3 crosslinking. d, e, Interactomes of BirA-IFITM3(Y20E) or empty vector control expressed in PDX2 B-ALL (d) and Jeko1 MCL (e) cells.f, $\mathbf{g}$, Validation of IFITM3-interacting proteins by anti-Flag co-immunoprecipitation (IP) in PDX2 cells transduced with Flag-IFITM3 or Flag-tagged empty vector, followed by western blotting for validation of interacting proteins, and STAT5

dominant-negative inhibition of IKZF1 relieved transcriptional repression of adhesion receptors, the normal expression and function of these receptors at the cell surface still required IFITM 3 and its ability to link integrins and adhesion receptors to the PI3K pathway. Loss of IFITM3, and reduced activity of the SRC kinase LYN and CD19-PI3K signalling, could broadly affect lipid rafts and short-circuit BCR signalling. Consistent with this scenario, the lipid-raft components cholesterol and ganglioside GM1 were markedly reduced in Ifitm $3^{-/-}$B cells (Fig. $4 \mathrm{a}$ ). In addition, BCR engagement increased membrane rigidity in IFITM3 ${ }^{+/+}$ MCL cells. However, membrane stiffening in response to BCR engagement was significantly reduced in IFITM3 ${ }^{-1-}$ MCL cells (Extended Data Fig. 8a). Consistent with defects in PI3K signalling, the ratios of PIP3 to the PI3K substrate PIP2 were reduced substantially in mouse fitm $^{-/-}$ B cells and patient-derived $I F I T M 3^{-1-}$ B-ALL cells. By contrast, overexpression of IFITM3 and IFITM3(Y20E) increased PIP3:PIP2 ratios by about 3-fold and 5.5-fold, respectively (Fig. 4b, c). To elucidate how IFITM 3 interacts with plasma membrane lipids, we probed 15 distinct lipid classes for binding to IFITM3 in vitro. IFITM3 bound selectively

to PIP3, but not to any of the other 14 lipids (Fig. 4d). Because IFITM3 function is required to retain normal PIP3 levels, we tested whether exogenous delivery of PIP3 could rescue Ifitm 3 deficiency in mice. Insertion of exogenous PIP3 into the cell membrane partially restored AKT and MYC activation and colony formation, but not CD19 and SRC phosphorylation or surface expression of lipid-raft-associated receptors (Extended Data Fig. 8b-d). Therefore, IFITM3 promotes PI3K signalling not only by increasing the amount of PIP3 but also by integrating PIP3 into signalling complexes in lipid rafts.

\section{Structural basis of IFITM3 binding to PIP3}

Proteins that contain a cluster of four or more basic residues located at the membrane-solution interface can laterally sequester PIP3, which does not require a PH domain or any other specific structure ${ }^{26}$. To map PIP3 binding to specific portions of IFITM3, we repeated lipid-binding assays with biotin-tagged fragments, including the $\mathrm{N}$ terminus, the first intramembrane $\alpha$-helix and the conserved intracellular loop. PIP3 


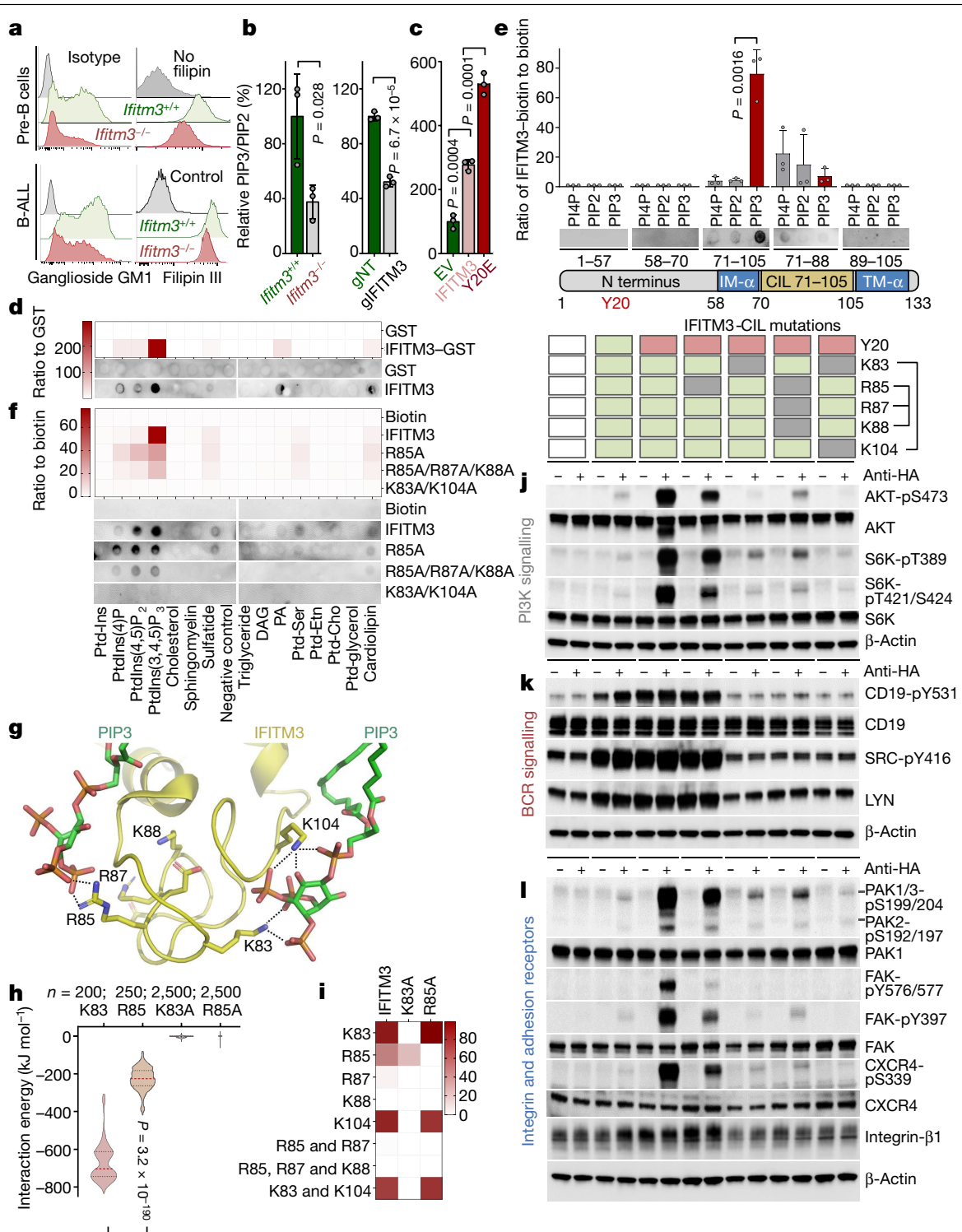

Fig. 4 | Structural basis of IFITM3-mediated regulation of PIP3 signalling from lipid rafts. a, Ganglioside GM1 and cholesterol (stained with filipin III) levels measured in $\mathrm{Ifitm}^{+/+}$and $\mathrm{Ifitm}^{-/-}$pre-B (top) and B-ALL (bottom) cells.

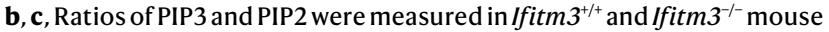
(b, left), and human B-ALL cells (PDX2) (b, right), and upon expression of empty vector, IFITM3 or IFITM3(Y2OE) (c). d, In vitro lipid-binding assays for glutathione-S-transferase-tagged IFITM3 (GST-IFITM3) and 15 lipid classes. Recombinant GST tag was used as baseline. e, Lipid-binding assays were performed for biotin-IFITM $3 \mathrm{~N}$ terminus (amino acids 1-57), the first intramembrane $\alpha$-helix (IM- $\alpha$ ) (amino acids 58-70), conserved intracellular loop (CIL) (amino acids 71-105) and transmembrane $\alpha$-helix (TM- $\alpha$ ) (amino acids 89-105).f, Lipid-binding assays to study interactions of PIP3 with biotinylated fragments of the IFITM 3 conserved intracellular loop that carry mutations of basic residues. $\mathrm{g}$-i, PIP3 binding to $\mathrm{K} 83$ and K104, and to R85, $\mathrm{R} 87$ and $\mathrm{K} 88$, basic residue patches (g). Dashed lines indicate PIP3 contacts

binding was detected only for the conserved intracellular loop (Fig. 4e), which contains five basic residues that are in close proximity in sequence and that spatially cluster into two distinct basic patches. To address why IFITM3 preferentially binds to PIP3 over PIP2, we performed multi-scale molecular dynamics simulations of IFITM3 in multi-component lipid bilayers that mimic the plasma membrane. All-atom molecular dynamics simulations revealed that the representative binding pose of PIP2 from its most populated conformation cluster showed a charge within $3.5 \AA$ A h, Interaction energies of PIP3 with basic residues K83 or R85 averaged over molecular dynamics simulation trajectories (median levels indicated by red dotted line) (Methods). i, Binding affinity for PIP3 as shown as a heat map of contact frequencies between PIP3 and each residue. $\mathbf{j}-\mathbf{I}$, Levels of AKT-pS473, AKT, S6K phosphorylated at T389 (S6K-pT389), S6K phosphorylated at T421 and S424 (S6K-pT421/S424), CD19-pY531, CD19, SRC-pY416, LYN, PAK1 and PAK3 phosphorylated at S199 and S204 (PAK1/3-pS199/204), PAK2 phosphorylated at S192 and S197 (PAK2-pS192/197), PAK1, FAK phosphorylated at Y397 (FAK-pY397), FAK phosphorylated at Y576 and Y577 (FAK-pY576/577), FAK, CXCR4 phosphorylated at S339

(CXCR4-pS339), CXCR4 and integrin- $\beta 1$ in PDX2 cells expressing empty vector, IFITM3-HA, IFITM3(Y2OE)-HA, and the R85A, K83A, R85A/R87A/K88A and $\mathrm{K} 83 \mathrm{~A} / \mathrm{K} 104 \mathrm{~A}$ mutants of IFITM3, upon IFITM 3 crosslinking with anti-HA antibodies. In $\mathbf{a}-\mathbf{f}, \mathbf{j}-\mathbf{l}, n=3$, For gel source data, see Supplementary Fig. 1 . In $\mathbf{b}, \mathbf{c}, \mathbf{e}, \mathbf{h}$, two-tailed $t$-test.

interaction with one single basic residue (R85) $\left(-213 \mathrm{~kJ} \mathrm{~mol}^{-1}\right)$, which was energetically weaker than the two charge interactions with PIP3 at K83 and $\mathrm{K} 104\left(-704 \mathrm{~kJ} \mathrm{~mol}^{-1}\right)(P=0.0008)$. Two-residue contacts were rare for PIP2 and more common for PIP3 ( $P=0.011)$ (Extended Data Fig. 9). To experimentally test the predicted function of the basic residues of the conserved intracellular loop, we performed PIP3 binding assays for biotinylated fragments of the IFITM3 conserved intracellular loop that carried mutations of these residues. The R85A and R85A/R87A/K88A 
mutants largely retained the ability to bind PIP3, which was abrogated for the K83A/K104A mutant (Fig. 4f). To understand the structural basis of this difference, we modelled the interaction of PIP3 with each of the mutants of the basic residues $(\mathrm{K} 83 \mathrm{~A} / \mathrm{K} 104 \mathrm{~A}, \mathrm{R} 85 \mathrm{~A} / \mathrm{R} 87 \mathrm{~A} / \mathrm{K} 88 \mathrm{~A}$ and R85A/R87A) using all-atom molecular dynamics simulations. PIP3 shows an interaction energy that is three times stronger with K83 and K104 than with R85, R87 and K88 (Fig. 4g-i). PIP3 makes dual-pronged salt-bridge interactions with K83 and K104, whereas it interacts only with R85 and not with R87 and K88 in the other basic patch. The $\mathrm{R} 87$ and $\mathrm{K} 88$ residues form intra-protein salt bridges with D92 and D86, respectively, and are not available for interaction with PIP3.

\section{IFITM3 amplifies PI3K, integrin and BCR signalling}

To experimentally validate this model, we globally identified changes in signal transduction that depend on $\mathrm{K} 83$ and $\mathrm{R} 85$ residues in human B-ALL and MCL B cells. The most prominent differences were enrichments for phosphorylation sites associated with PI3K, BCR and adhesion receptor signalling (Extended Data Fig. 10a, b). Although most phosphorylation events induced by membrane-bound IFITM3(Y20E) were lost upon mutation of K83, this was not the case for substitution of the $\mathrm{R} 85$ residue (Extended Data Fig. 10c, d). Proximity ligation assays revealed IFITM3-dependent recruitment of multiple proteins in the $\mathrm{PI} 3 \mathrm{~K}, \mathrm{BCR}$ and integrin signalling pathways. However, almost all of these interactions were lost upon mutation of K83 (Extended Data Fig. 10e-h). Biochemical studies revealed that crosslinking of IFITM3(Y2OE) resulted in massive activation of PI3K and integrin receptor pathways, whereas CD19 and SRC were constitutively phosphorylated in the presence of IFITM3(Y2OE) (Fig. 4j-l). The R85A mutation had only minor effects, whereas the substitution of K83 almost entirely abolished IFITM3-dependent signal transduction. In contrast to the triple-mutant IFITM3(R85A/R87A/K88A), the IFITM3(K83A/K104A) double mutant lost all activity in PI3K, BCR and adhesion receptor pathways (Fig. $4 \mathrm{j}-\mathrm{l}$ ).

In summary, we have revealed a function for IFITM3 as a PIP3 scaffold in the amplification of PI3K signalling. In Ifitm $3^{-/-}$B cells, lipid rafts were depleted of PIP3, which resulted in marked defects in lipid-raft-associated BCR and integrin receptor signalling. IFITM3-dependent amplification of PI3K signalling, acting-in partdownstream of the BCR and adhesion receptors, is critical for the rapid expansion of $B$ cells with high affinity to antigen. In addition, multiple oncogenes depend on IFITM3 to assemble PIP3-dependent signalling complexes and amplify PI3K signalling for malignant transformation.

\section{Online content}

Any methods, additional references, Nature Research reporting summaries, source data, extended data, supplementary information, acknowledgements, peer review information; details of author contributions and competing interests; and statements of data and code availability are available at https://doi.org/10.1038/s41586-020-2884-6.

1. Bailey, C. C., Zhong, G., Huang, I. C. \& Farzan, M. IFITM-family proteins: the cell's first line of antiviral defense. Annu. Rev. Virol. 1, 261-283 (2014).

2. Diamond, M. S. \& Farzan, M. The broad-spectrum antiviral functions of IFIT and IFITM proteins. Nat. Rev. Immunol. 13, 46-57 (2013).

3. Brass, A. L. et al. The IFITM proteins mediate cellular resistance to influenza A H1N1 virus, West Nile virus, and dengue virus. Cell 139, 1243-1254 (2009).

4. Zhang, Y. et al. Interferon-induced transmembrane protein 3 genetic variant rs12252-C associated with disease severity in coronavirus disease 2019. J. Infect. Dis. 222, 34-37 (2020).
5. Zhao, X. et al. Identification of residues controlling restriction versus enhancing activities of IFITM proteins on entry of human coronaviruses. J. Virol. 92, e01535-17 (2018).

6. Jia, R. et al. The N-terminal region of IFITM 3 modulates its antiviral activity by regulating IFITM3 cellular localization. J. Virol. 86, 13697-13707 (2012).

7. Chesarino, N. M., McMichael, T. M., Hach, J. C. \& Yount, J. S. Phosphorylation of the antiviral protein interferon-inducible transmembrane protein 3 (IFITM3) dually regulates its endocytosis and ubiquitination. J. Biol. Chem. 289, 11986-11992 (2014).

8. Everitt, A. R. et al. IFITM3 restricts the morbidity and mortality associated with influenza. Nature 484, 519-523 (2012).

9. Makvandi-Nejad, S. et al. Lack of truncated IFITM3 transcripts in cells homozygous for the rs12252-C variant that is associated with severe influenza infection. J. Infect. Dis. 217, 257-262 (2018).

10. Kohn, A. Early interactions of viruses with cellular membranes. Adv. Virus Res. 24, 223-276 (1979).

11. Liu, S. Y., Sanchez, D. J. \& Cheng, G. New developments in the induction and antiviral effectors of type I interferon. Curr. Opin. Immunol. 23, 57-64 (2011).

12. Li, D. et al. KLF4-mediated negative regulation of IFITM3 expression plays a critical role in colon cancer pathogenesis. Clin. Cancer Res. 17, 3558-3568 (2011).

13. Zhang, D., Wang, H., He, H., Niu, H. \& Li, Y. Interferon induced transmembrane protein 3 regulates the growth and invasion of human lung adenocarcinoma. Thorac. Cancer $\mathbf{8}$ 337-343 (2017).

14. Spence, J. S. et al. IFITM3 directly engages and shuttles incoming virus particles to lysosomes. Nat. Chem. Biol. 15, 259-268 (2019).

15. Harvey, R. C. et al. Identification of novel cluster groups in pediatric high-risk B-precursor acute lymphoblastic leukemia with gene expression profiling: correlation with genomewide DNA copy number alterations, clinical characteristics, and outcome. Blood 116, 4874-4884 (2010)

16. Witkowski, M. T. et al. Conserved IKAROS-regulated genes associated with B-progenitor acute lymphoblastic leukemia outcome. J. Exp. Med. 214, 773-791 (2017).

17. Fujimoto, M. et al. CD19 regulates Src family protein tyrosine kinase activation in B lymphocytes through processive amplification. Immunity 13, 47-57 (2000).

18. Okkenhaug, K. et al. Impaired B and T cell antigen receptor signaling in p110 $\mathrm{PI}$ 3-kinase mutant mice. Science 297, 1031-1034 (2002).

19. Engel, P. et al. Abnormal B lymphocyte development, activation, and differentiation in mice that lack or overexpress the CD19 signal transduction molecule. Immunity 3, 39-50 (1995).

20. Chan, L. N. et al. Metabolic gatekeeper function of B-lymphoid transcription factors. Nature 542, 479-483 (2017).

21. Wang, Y. et al. The physiologic role of CD19 cytoplasmic tyrosines. Immunity 17, 501-514 (2002).

22. Phelan, J. D. et al. A multiprotein supercomplex controlling oncogenic signalling in lymphoma. Nature 560, 387-391 (2018).

23. Kläsener, K., Maity, P. C., Hobeika, E., Yang, J. \& Reth, M. B cell activation involves nanoscale receptor reorganizations and inside-out signaling by Syk. eLife 3, e02069 (2014).

24. Schwickert, T. A. et al. Stage-specific control of early B cell development by the transcription factor lkaros. Nat. Immunol. 15, 283-293 (2014).

25. Joshi, I. et al. Loss of Ikaros DNA-binding function confers integrin-dependent survival on pre-B cells and progression to acute lymphoblastic leukemia. Nat. Immunol. 15, 294-304 (2014).

26. McLaughlin, S. \& Murray, D. Plasma membrane phosphoinositide organization by protein electrostatics. Nature 438, 605-611 (2005).

Publisher's note Springer Nature remains neutral with regard to jurisdictional claims in published maps and institutional affiliations.

(c) The Author(s), under exclusive licence to Springer Nature Limited 2020 , corrected publication 2021

${ }^{1}$ Center of Molecular and Cellular Oncology, Yale Cancer Center, Yale School of Medicine, New Haven, CT, USA. ${ }^{2}$ Department of Computational and Quantitative Medicine, City of Hope Comprehensive Cancer Center, Duarte, CA, USA. ${ }^{3}$ Department of Systems Biology, City of Hope Comprehensive Cancer Center, Duarte, CA, USA. ${ }^{4}$ Department of Laboratory Medicine, University of California San Francisco, San Francisco, CA, USA. ${ }^{5}$ Koch Institute for Integrative Cancer Research, Massachusetts Institute of Technology, Cambridge, MA, USA. ${ }^{6}$ Medical Research Council Laboratory for Molecular Cell Biology, University College London, London, UK. 'Department of Pathology and Laboratory Medicine, Children's Hospital of Philadelphia and Perelman School of Medicine, University of Pennsylvania, Philadelphia, PA, USA. ${ }^{8}$ Department of Immunology and Microbiology, The Scripps Research Institute, Jupiter, FL, USA. ${ }^{9}$ Department of Cancer Biology, Lerner Research Institute, Cleveland Clinic, Cleveland, OH, USA. ${ }^{10}$ Dana Farber Cancer Institute, Boston, MA, USA. "Harvard Medical School, Boston, MA, USA. ${ }^{12}$ Department of Biological Engineering, Massachusetts Institute of Technology, Cambridge, MA, USA. ${ }^{13}$ Department of Medicine, Washington University School of Medicine in St Louis, St Louis, MO, USA. ${ }^{14}$ Department of Molecular Microbiology, Washington University School of Medicine in St Louis, St Louis, MO, USA. ${ }^{15}$ Department of Pathology and Immunology, Washington University School of Medicine in St Louis, St Louis, MO, USA. ${ }^{16}$ Department of Immunobiology, Yale University, New Haven, CT, USA. ${ }^{\varpi_{e}}$-mail: markus. muschen@yale.edu 


\section{Methods}

No statistical methods were used to predetermine sample size. The experiments were not randomized and investigators were not blinded to allocation during experiments and outcome assessment.

\section{Analysis of patient gene expression and outcome data}

Gene expression microarray data from three large cohorts of patients with pre-B ALL were downloaded from GSE5314 ${ }^{27}$ (the Eastern Cooperative Oncology Group (ECOG) Clinical Trial E2993), GSE11877 ${ }^{28}$ (the Children's Oncology Group (COG) Clinical Trial P9906), St.Jude Research Hospital paediatric $\mathrm{ALL}^{29}$ (http://www.stjuderesearch.org/site/data/ ALL3/). In ECOG E2993, pretreatment bone marrow or peripheral blood samples were obtained at diagnosis before any treatment from 83 patients with $\mathrm{Ph}^{+} \mathrm{B}$-ALL enrolled in the Medical Research Council (MRC) UKALLXII/Eastern Cooperative Oncology Group (ECOG) E2993 phase III trial. In data set from St. Jude childhood, 15 cases of $\mathrm{Ph}^{+} \mathrm{B}-\mathrm{ALL}$ were selected from the original 327 diagnostic bone marrow aspirates. Minimal residual disease (MRD) data were available for patients with paediatric high-risk B-ALL (COG P9906).IFITM3 expression levels were measured in patients with either a MRD positive $\left(\mathrm{MRD}^{+}\right)$status or a MRD negative (MRD') status. In COG P9906 clinical outcome, expression profiles were obtained in pretreatment leukaemic samples from 207 uniformly treated children with high-risk $\mathrm{ALL}^{30}$, a risk category largely defined by pretreatment clinical characteristics. Patients had MRD tested by flow cytometry with two combinations (CD20/CD10/ CD45 or CD9/CD19/CD34/CD45), and were defined as MRD positive or MRD negative at the end of induction therapy (day 29) using a threshold of $0.01 \%$ as previously described ${ }^{31}$. Then, RNA was purified from 207 pretreatment diagnostic samples with more than $80 \%$ blasts (131 bone marrow, 76 peripheral blood) and subjected to microarrays. Log-rank test was used to assess statistical significance.

\section{Primary human samples and cell lines}

Patient samples (Supplementary Table 7) were sourced ethically from patients who gave informed consent, and were in compliance with the internal review boards of the Beckman Research Institute of City of Hope. We have complied with all relevant ethical regulations. Patient samples were harvested from biopsy of bone marrow from patients with ALL at the time of diagnosis or relapse. All samples were transplanted into sublethally irradiated NOD.Cg-Prkd $c^{\text {scid }} / l 2 r g^{\text {tmlWjl }} / \mathrm{SzJ}$ mice (NSG mice, The Jackson Laboratory) through tail-vein injection. After samples were collected, patient-derived primary human pre-B ALL xenografts were cultured on OP9 stroma in Alpha Minimum Essential Medium (MEM $\alpha$; Life Technologies) with GlutaMAX containing 20\% fetal bovine serum (FBS), $100 \mathrm{IU} \mathrm{ml}^{-1}$ penicillin, $100 \mathrm{\mu g} \mathrm{ml}^{-1}$ streptomycin and $1 \mathrm{mM}$ sodium pyruvate. The human cell lines (Supplementary Table 8) were cultured in RPMI-1640 (GIBCO) with GlutaMAX containing $20 \% \mathrm{FBS}, 100 \mathrm{IU} \mathrm{ml}^{-1}$ penicillin, $100 \mathrm{\mu g} \mathrm{ml}^{-1}$ streptomycin at $37^{\circ} \mathrm{C}$ in a humidified incubator with $5 \% \mathrm{CO}_{2}$. All human primary samples and cell lines were tested negative for mycoplasma by detection kit (MycoAlert PLUS, LONZA).

\section{Genetic mouse models}

Genetic mouse models used in this study are listed in Supplementary Table 9. NOD.Cg-Prkdc ${ }^{\text {scid }} / l 2 r^{\text {tmlWjl }} / \mathrm{SzJ}$ (NSG) mice were purchased from Jackson Laboratory. Ifitm $3^{\text {tm1Masu }}$ (ref. ${ }^{32}$ ) mice were backcrossed to the $\mathrm{C} 57 \mathrm{BL} / 6 \mathrm{~J}$ background for more than eight generations.

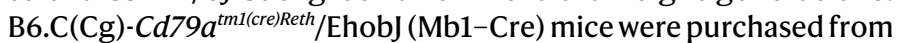
Jackson Laboratory. B6.129S2-lghm ${ }^{\text {tmlcgn }} / \mathrm{J}(\mu \mathrm{MT})$ mice were purchased from Jackson Laboratory. C;129S4-Pten ${ }^{\text {tmlHwu}} / J\left(\right.$ Pten $\left.^{\mathrm{f} / \mathrm{f}}\right)$ mice were purchased from Jackson Laboratory. For mice bred in-house, littermates of the same sex were randomized to experimental groups. For the in vivo leukaemia initiation assay, 8- to 10-week-old female NSG mice were randomly allocated before injection. To generate a model for pre-leukaemic B cell precursors expressing BCR-ABL1, LSL-Bcr ${ }^{+/ B C R-A B L 1}$ mice ${ }^{33}$ were crossed with $M b 1$-cre strain $(M b 1$-cre $\times$ LSL-Bcr/BCR-ABL1 $)$ for excision of a stop-cassette in early pre-B cells. For in vivo oncogenic priming assay with $M b 1$-cre $\times \mathrm{LSL}-\mathrm{Bcr}^{+/ B C R-A B L 1} \mathrm{~B}$ cell precursors, 8- to 10-week-old female NSG mice were randomly allocated before injection. Temperatures of $18-23^{\circ} \mathrm{C}$ with $40-60 \%$ humidity were maintained with 14 -h light/10-h dark cycle. The following scores were considered as end-point: (1) a failure to eat food or drink water for $24 \mathrm{~h}$; (2) a failure to make normal postural adjustments or to display normal behaviour; and (3) when tumour burden reached $1.5 \mathrm{~cm} \times 1.5 \mathrm{~cm} \times 1.5 \mathrm{~cm}$ (tumour ulceration was not expected). If a mouse lost $25 \%$ of its initial body weight (or reached $16 \mathrm{~g}$ of body weight, regardless of the initial weight), or if we observed a weight loss of $15 \%$ on 2 sequential weight measurements, we euthanized the mouse immediately. All mouse experiments were subject to institutional approval by the Beckman Research Institute of City of Hope Animal Care and Use Committee.

\section{Mouse primary and leukaemia cells}

Bone marrow cells from 6-10-week-old mice were collected by flushing cavities of the femur and tibia with chilled PBS, followed by filtering through $40-\mu \mathrm{m}$ strainer to yield a single-cell suspension. Spleen or thymus cells were directly extracted by forcing tissues through a $40-\mu \mathrm{m}$ strainer into chilled PBS. Filtered cells were further incubated with lysis buffer (RBC Lysis Buffer, BioLegend) to lyse erythrocytes. After washing with PBS, cells were subjected to further experiments. For IL-7-dependent pre-B cell culture, bone marrow cells were collected and cultured in Iscove's modified Dulbecco's medium (IMDM) (GIBCO) with GlutaMAX containing 20\% FBS, $50 \mu \mathrm{M} 2$-mercaptoethanol, 100 $\mathrm{IU} \mathrm{ml} \mathrm{I}^{-1}$ penicillin, $100 \mu \mathrm{g} \mathrm{ml}^{-1}$ streptomycin in the presence of $10 \mathrm{ng}$ $\mathrm{ml}^{-1}$ recombinant mouse IL-7 (Peprotech). For the $B C R-A B L 1$ leukaemia model, pre-B cells were retrovirally transformed with $B C R-A B L 1$ and then IL-7 was removed to select the transduced cells. ras $^{G 12 D} \mathrm{ALL}$ cells were selected with puromycin (GIBCO) and were maintained with IL-7-supplemented IMDM.

\section{Retroviral and lentiviral transduction}

Retroviral supernatant was generated by cotransfection of HEK 293FT cells with retroviral constructs together with pHIT60 (gag-pol) and pHIT123 (for mouse) or pHIT456 (for human ecotropic envelope) using Lipofectamine 2000 (Invitrogen). Lentiviral supernatant for CRISPR-mediated gene editing was produced by cotransfection of HEK 293FT cells with lentiviral constructs together with pCDNL/BH and EM140. Transfected HEK 293FT cells were cultured in high-glucose Dulbecco's modified Eagle's medium (DMEM) (GIBCO) with GlutaMAX containing $10 \%$ fetal bovine serum, $100 \mathrm{IU} \mathrm{ml}^{-1}$ penicillin, $100 \mu \mathrm{g} \mathrm{ml}^{1}$ streptomycin, $25 \mathrm{mmol} \mathrm{I}^{-1} \mathrm{HEPES}, 1 \mathrm{mmol}^{-1}$ sodium pyruvate and 0.1 $\mathrm{mmol}^{-1}$ non-essential amino acids for $16 \mathrm{~h}$. After sodium butyrate $(10 \mathrm{mM})$ induction for $8 \mathrm{~h}$, the virus supernatant was collected and filtered through a $0.45-\mu \mathrm{m}$ filter. The virus-containing supernatants were loaded by centrifugation $\left(2,000 \mathrm{~g}, 90 \mathrm{~min}\right.$ at $\left.32^{\circ} \mathrm{C}\right)$ on $50 \mu \mathrm{g}$ $\mathrm{ml}^{-1}$ retronectin (Takara)-coated non-tissue culture 6-well plates. For retroviral transduction, $3-5 \times 10^{6}$ cells were transduced per well by centrifugation at $600 \mathrm{~g}$ for $30 \mathrm{~min}$ in the appropriate culture medium and maintained at $37^{\circ} \mathrm{C}$ at $5 \% \mathrm{CO}_{2}$ for $48 \mathrm{~h}$. For lentiviral transduction, $3-5 \times 10^{6}$ cells per well were centrifuged at $600 \mathrm{~g}$ for $30 \mathrm{~min}$ in the presence of lentiviral supernatant and maintained at $37^{\circ} \mathrm{C}$ at $5 \% \mathrm{CO}_{2}$. The lentiviral supernatant was replaced with fresh medium the next day.

\section{Western blotting}

PBS-washed cells were lysed in CelLytic buffer (Sigma-Aldrich) supplemented with $1 \%$ protease inhibitor cocktail (Roche Diagnostics), $1 \%$ phosphatase inhibitor cocktail (EMD Millipore) and 1 mM PMSF on ice. A total of $10 \mu \mathrm{g}$ of cell lysates was separated on mini-precast gels (Bio-Rad) and transferred onto nitrocellulose membranes (Bio-Rad). Membranes were probed with the appropriate primary antibodies. 
Membranes were then incubated with alkaline-phosphatase conjugated secondary antibodies (Invitrogen) and chemiluminescent substrate (Invitrogen) and were further detected by film exposure, UVP BioSpectrum 810 Imaging System (Thermo Fisher Scientific) or by using the ChemiDoc MP Imaging System (BioRad). Antibodies used in this study are provided in Supplementary Table 10, and were used at 1:750 to 1:1,000 dilution in blocking buffer.

\section{Flow cytometry}

PBS-washed cells were blocked with Fc blocker for 10 min on ice and then stained with the appropriate antibodies listed in Supplementary Table 10, or with isotype control, for $25 \mathrm{~min}$ on ice. Cells were then washed and resuspended in chilled PBS containing 0.75 $\mu \mathrm{g} \mathrm{ml}^{-1}$ of DAPI to exclude dead cells. Acquisition was performed by LSRFortessa flow cytometer (BD Biosciences) with BD FACSDIVA software. The fluorescence-based cell sorting was performed by FACSAria II (BD Biosciences) with BD FACSDIVA software. FACS data were analysed with FlowJo software (FlowJo). For apoptosis analyses, annexin $V$ and 7-AAD (BD Biosciences) were used. For cell cycle analysis, the BrdU flow cytometry kit (BD Biosciences) was used according to manufacturer's instructions. For intracellular staining of cytoplasmic proteins, cells were first stained for cell surface antigens and subsequently fixed in fixation and permeabilization solution (BD Biosciences) containing 4\% paraformaldehyde and the detergent saponin. Cells were then washed and resuspended in Perm/Wash Buffer (BD Biosciences) and stained with the appropriate antibodies. For statistical quantification, data were plotted with GraphPad Prism 7 or SigmaPlot. FACS antibodies, listed in Supplementary Table 10, were pre-diluted 1:5-1:10. Two $\mu$ l of diluted antibody was added to 1-2 million cells per $50 \mu \mathrm{l}$ in PBS for final dilution of 1:50 for human cells or 1:200 for mouse cells.

\section{Pharmacological inhibitors and reagents}

Imatinib was purchased from LC Laboratories. Stock solutions were prepared in sterile water at $10 \mathrm{mmol} \mathrm{I}^{-1}$ and administered at $10 \mu \mathrm{mol}$ $\mathrm{I}^{-1}$. Dasatinib was purchased from SelleckChem. Stock solutions were prepared in sterile DMSO at $25 \mathrm{mmoll}^{-1}$ and administered at $25 \mathrm{nmoll}^{-1}$ (Supplementary Table 11).

\section{Analysis of chromatin immunoprecipitation with sequencing data}

IKZF1 chromatin immunoprecipitation with sequencing (ChIP-seq) was performed as previously described ${ }^{34}$ with a patient-derived human B-ALL xenograft cell line (LAX2), which expresses wild-type full-length IKZF1 and no detectable level of the dominant-negative IKZF1 isoform. ChIP-seq tracks (GSE58825) for the IKZF1 antibody in LAX2 on IFITM3 gene promoter regions are shown in the bottom of Extended Data Fig. 1m. The $y$ axis represents the normalized number of reads per million reads for peak summit for each track. ChIP-seq tracks (GSE86897) for the enrichment of RNAPII and H3K4me3 at the Ifitm3 locus in pre-B cells from $I k z f 1^{\text {exonsfl/fl }}$ mice upon Cre-mediated deletion of $I k z f 1$ are shown in the top of Extended Data Fig. $1 \mathrm{~m}$. The ChIP-seq peaks were called by the MACS peak-caller by comparing read density in the ChIP experiment relative to the input chromatin control reads, and are shown as bars under each wiggle track. Gene models are shown in UCSC genome browser hg19. Integrative Genomics Viewer (IGV) was used to visualize ChIP-seq tracks.

\section{Inducible expression of IKZF1}

Human Philadelphia chromosome-positive $\left(P h^{+}\right)$pre-B ALL cells (BV173) carrying deletions of $I K Z F 1$ were transduced with pRetroX-Tet3G-Neo. Neomycin-resistant cells were transduced with pRetroX-TRE3G-wild-type IKZF1-Puro. Expression of wild-type IKZF1 in puromycin-resistant cells was initiated by treatment with $1 \mathrm{\mu g} \mathrm{ml}^{-1}$ of doxycycline (TetOn).

\section{Inducible reconstitution of CD19}

For inducible CD19 reconstitution, mouse CD19 was fused to the ligand-binding domain of a mutant oestrogen receptor $\left(E R^{\mathrm{T} 2}\right)$ at the $C$ terminus of CD19. BCR-ABL1-transformed mouse B-lineage ALL cells were retrovirally transduced with MSCV CD19-ER ${ }^{\mathrm{T} 2}$-Puro. Puromycin-resistant cells were treated with 4-hydroxytamoxifen (4-OHT) (Sigma-Aldrich) or vehicle control to induce reconstitution of CD19.

\section{Cell viability assay}

One hundred thousand BCR-ABL1-transformed mouse B-lineage ALL cells were seeded in a volume of $100 \mu \mathrm{l}$ in medium in 1 well of a 96-well plate (BD Biosciences). Imatinib (LC Laboratories) was added at the indicated concentration in a total volume of $150 \mu$ l. After culturing for 3 days, $15 \mu$ l of resazurin (R\&D) was added into each well and incubated for $4 \mathrm{~h}$ at $37^{\circ} \mathrm{C}$. Medium without cells was used as blank. The fluorescence was read at $535 \mathrm{~nm}$ and the reference wavelength was $590 \mathrm{~nm}$. Relative viability was calculated using baseline values of vehicle-treated cells as a reference.

\section{Colony-forming assay}

The methylcellulose colony-forming assays were performed with 10,000 cells. Cells were resuspended in mouse MethoCult medium (without cytokines for BCR-ABL1-transformed cells; with IL-7 for $N R A S^{G 12 D}$-expressing cells) and cultured on 3-cm-diameter dishes, with an extra water supply dish to prevent evaporation. Colonies were imaged and counted after seven days using GelCount (Oxford Optronix), Olympus IX71 microscope and Q-Capture pro 7.

\section{In vitro IFITM3-HA crosslinking}

Eight million patient-derived B-ALL cells per sample were resuspended into complete medium and treated with either $2.5 \mu \mathrm{g} \mathrm{ml}^{-1}$ polyclonal anti-HA (Abcam) or isotype control for the indicated times. $\mathrm{F}(\mathrm{ab})$ fragments for the anti-HA antibody were generated by using the $F(a b)$ preparation kit following the manufacturer's instructions (Thermo Fisher Scientific). F(ab) fragmentation was confirmed by Criterion TGX Stain-Free Precast Gels (Bio-Rad).

\section{Measurement of intracellular calcium mobilization}

In Extended Data Fig. 2f, 1 million fresh splenocytes were incubated with the $4 \mu \mathrm{M}$ Rhod-2-AM Ca ${ }^{2+}$-binding dye (Thermo Fisher Scientific) for 15 min at room temperature in dark. Cells were then resuspended in PBS and maintained at $37^{\circ} \mathrm{C}$, and $\mathrm{Ca}^{2+}$ response was induced by adding $10 \mu \mathrm{g}$ $\mathrm{ml}^{-1}$ of polyclonal anti-IgM (Southern Biotech) at $50 \mathrm{~s}$ after acquisition of background fluorescence. Intracellular $\mathrm{Ca}^{2+}$ mobilization in response to crosslinked IgM was measured up to 300 s by flow cytometry. In Extended Data Fig. $4 \mathrm{c}, 1 \times 10^{6}$ viable Jurkat cells were incubated with the $4 \mu \mathrm{M}$ Fluo4-AM Ca ${ }^{2+}$-binding dye (Thermo Fisher Scientific) for 15 min at room temperature in dark. Cells were then resuspended in PBS and maintained at $37^{\circ} \mathrm{C}$, and $\mathrm{Ca}^{2+}$ response was induced by adding 10 $\mu \mathrm{g} \mathrm{ml}^{-1}$ of monoclonal (OKT3) purified NA/LE anti-human CD3 (Biolegend) at 50 s after acquisition of background fluorescence. Intracellular $\mathrm{Ca}^{2+}$ mobilization in response to crosslinked IFITM3 was measured up to 300 s by flow cytometry. The same procedure was performed for Extended Data Fig. $6 \mathrm{f} ; 1 \times 10^{6}$ viable Jeko1 cells were incubated with the $4 \mu$ M Fluo4-AM (Thermo Fisher Scientific). $\mathrm{Ca}^{2+}$ response was induced by adding $10 \mu \mathrm{g} \mathrm{ml}^{-1}$ of polyclonal $\mathrm{F}\left(\mathrm{ab}^{\prime}\right)_{2}$ anti-human $\mu$ chain (Jackson Immunoresearch).

\section{Homotypic aggregation assay}

Ifitm $3^{-1-}$ B-ALL cells expressing C-terminal HA-tagged wild-type IFITM3, IFITM3 mutant vector (Y2OE) or an empty vector were incubated with $2.5 \mathrm{\mu g} \mathrm{ml}^{-1}$ of monoclonal (1D3) purified LEAF anti-mouse CD19 (Biolegend) or $2.5 \mu \mathrm{g} \mathrm{ml}^{-1}$ polyclonal anti-HA (Abcam) or isotype controls 
at $37^{\circ} \mathrm{C}$ at $5 \% \mathrm{CO}_{2}$. After culturing for $24 \mathrm{~h}$, homotypic aggregation was visualized using light microscopy.

\section{In vivo oncogenic priming assay with $M b 1-c r e ; L S L-B c r^{+/ B C R-A B L 1}$ B cell precursors}

To generate a model for pre-leukaemic $B$ cell precursors expressing BCR-ABL1, BCR-ABL1 knock-in mice were crossed with Mb1-Cre strain $\left(M b 1-c r e \times\right.$ LSL-Bcr $\left.{ }^{+/ B C R-A B L 1}\right)$ for excision of a stop cassette in early pre-B cells. Bone marrow cells collected from $M b 1$-cre $\times$ LSL-Bcr $r^{+/ B C R-A B L 1}$ mice, cultured in the presence of $10 \mathrm{ng} \mathrm{ml}^{-1}$ recombinant mouse IL-7 (Peprotech). IL-7 dependent pre-leukaemic B cells were labelled with retroviral firefly luciferase and selected by blasticidin for 7 days. After selection, cells were further transduced with MSCV-IFITM3-HA-IRES-Puro, MSCV-IFITM $3^{\mathrm{Y} 20 \mathrm{E}}$-HA-IRES-Puro or empty vector and selected by puromycin for 3 days.Viable cells $\left(1 \times 10^{7}\right)$ were injected via the tail vein into sublethally irradiated ( $200 \mathrm{cGy})$ NSG recipient mice. In vivo expansion and leukaemia burden were monitored by luciferase bioimaging (IVIS 100 bioluminescence and optical imaging system; Xenogen) at the indicated time points. In brief, D-luciferin (Promega) dissolved in PBS was injected intraperitoneally at a dose of $2.5 \mathrm{mg}$ per mouse $15 \mathrm{~min}$ before measuring luminescence. All mice were anaesthetized by $5 \%$ isoflurane and continued during detection of light emission with $2 \%$ isoflurane introduced through a nose cone. A mouse was euthanized when they showed signs of leukaemia burden such as a hunched back, weight loss and inability to move. Kaplan-Meier survival analysis was performed using GraphPad Prism 7 (GraphPad Software) to compare overall survival. Mantel-Cox log-rank test was used as statistical analysis using GraphPad Prism 7 (Supplementary Table 4).

\section{Cell adhesion assay}

Ifitm $^{+/+}$or Ifitm $^{-/-} B C R-A B L 1$ B-ALL cells were transduced with MSCV-IK6-IRES-GFP or MSCV-IRES-GFP as negative control. GFP cells $\left(1 \times 10^{5}\right)$ sorted by FACS were cultured on $1.5 \times 10^{5}$ OP9 stroma on a 6-well plate with IMDM-GlutaMAX containing $20 \% \mathrm{FBS}, 50 \mu \mathrm{M}$ 2-mercaptoethanol, $100 \mathrm{IU} \mathrm{ml}^{-1}$ penicillin and $100 \mathrm{\mu g} \mathrm{ml}^{-1}$ streptomycin. For calculation of the ratio of adherent cells to nonadherent cells, nonadherent cells were collected and counted with the trypan blue exclusion method using the Countess II FL Automated Cell Counter (Life Technologies). After washing the plate with PBS twice, adherent cells were detached with trypsinization and $\mathrm{GFP}^{+} \mathrm{B}-\mathrm{ALL}$ cells and were counted with the trypan blue exclusion method using the Countess II FL Automated Cell Counter (Life Technologies) and ratios were calculated.

\section{Adoptive transfer of purified B cells into $\mu \mathrm{MT}$ mice}

For the adoptive transfer of B cells, splenocytes from 7- to 10-week-old Ifitm $^{+/+}$or Ifitm $3^{-/-}$mice were negatively selected by MojoSort Mouse Pan B Cell Isolation Kit II (Miltenyi Biotec) using immunomagnetic beads against CD3, CD4, CD8a, CD11c, CD49b, Ly6G/Ly6C (GR1) and TER119. Ten million flow-sorted splenic $B$ cells were intravenously injected into $\mu \mathrm{MT}$ mice (B6.129S2-lghm ${ }^{\text {tmlcgn }} / \mathrm{J}$ ) that lack the $\mathrm{C} \mu$ exon and mature $B$ cell development as a result of defective surface IgM expression. Reconstitution of donor B cells was determined by flow cytometry 20 days after injection. Recipient $\mu \mathrm{MT}$ mice were immunized with the hapten NP coupled to a carrier protein (KLH) on day 0 and day 7 , and spleens were collected on day 12 .

\section{Immunization and immunohistology}

Ifitm $^{+/+}$and Ifitm $^{-/-}$littermate mice were immunized with $0.5 \mathrm{mg}$ $\mathrm{NP}-\mathrm{KLH}$ (Biosearch Technologies) in alum (Sigma-Aldrich) intraperitoneally. Seven days later, mice were immunized a second time for five days. Spleens were isolated at day 12 after immunization. The spleen was embedded in optimum cutting temperature compound and 5 - $\mu \mathrm{m}$-thick cryosections were used for staining. Sections were fixed with acetone for $10 \mathrm{~min}$, and nonspecific antigens were blocked in DPBS containing $2 \% \mathrm{FBS}$ for $15 \mathrm{~min}$. Sections were stained with 1:200 diluted polyclonal
(RA3-6B2) anti-CD45R (B220, BD Biosciences), monoclonal anti-mouse CD3 (17A2, BioLegend) antibodies and biotinylated peanut agglutinin (B-1075, Vector Laboratories) for $45 \mathrm{~min}$. Sections were washed and further stained with Alexa Fluor 647 streptavidin (BioLegend) antibody for $45 \mathrm{~min}$. All antibodies were diluted 1:100 to their original concentration in blocking buffer. After washing with blocking buffer, sections were mounted in ProLong Diamond Antifade Mountant (ThermoFisher Scientific). Images were acquired on a ZEISS LSL 880 confocal microscope and analysed on ZEN 2.3 (Zeiss) software.

\section{ELISA measurements}

For determination of the concentrations of immunoglobulin isotypes in sera, ELISAs were carried out according to the manufacturer's protocol (Ig Isotyping Mouse Instant ELISA Kit for IgG1, IgG2b and IgM) (Supplementary Table 11). NP-specific antibodies were measured by ELISA using $10 \mu \mathrm{g} / \mathrm{ml}$ of NP(24)-BSA (Biosearch Technologies) as the coating reagent. NP-specific IgM and IgG1 was detected using goat anti-mouse IgM and IgG1 Fc-specific antibodies conjugated to horseradish peroxidase and developed with tetramethylbenzidine (Sigma). Optical densities were determined by using an ELISA reader at $450 \mathrm{~nm}$ (SpectraMax M3, Molecular Devices).

\section{RNA-sequencing analysis}

Total mRNA from $/$ fitm $^{+/ /+}$and $I$ fitm $3^{-/-} B C R-A B L 1$ or $N R A S^{G 12 D}$ B-ALL cells was extracted using RNeasy Kit (Qiagen) according to the manufacturer's instructions. Sequencing was performed on an Illumina Hiseq 2500 (Illumina) instrument using the TruSeq SR Cluster Kit v.4-cBot-HS (Illumina) with v.4 chemistry. Quality control of RNA-sequencing reads was performed using FastQC. For analysis, raw sequence reads were mapped to the mouse genome ( $\mathrm{mm} 10)$ using STAR v.2.5.3 ${ }^{35}$, and the frequency of genes was counted using featureCounts v.1.5.1 ${ }^{36}$. The raw counts were then normalized using the trimmed mean of $M$ values method and compared using Bioconductor package ' edgeR $^{\prime 37}$. Reads per kilobase per million (RPKM) mapped reads were also calculated from the raw counts. For differential expression analysis, transcripts were quantified using Salmon v.1.1.0 against gencode GRCm38 v.M24 transcript annotations; normalization and statistical analysis were done in $\mathrm{R}$ using DESeq2 v.1.28.1. Differentially expressed genes were identified if RPKM $\geq 1$ in at least one sample, fold change $\geq 2$ and $P \leq 0.05$ (Extended Data Fig. 3b). RPKM data were later used in the gene set enrichment analysis (GSEA). GSEA was performed using the DOSE package in $\mathrm{R}^{38}$; genes were ranked by $\log _{2}$-transformed fold change; and gene sets were obtained from MSigDB or from internal data, as indicated.

\section{CRISPR-mediated gene deletion}

All lentiviral constructs expressing Cas9 nuclease and guide RNA were purchased from Transomic Technologies. For gene deletion, cells were transduced with pTOL-hCMV-Tet3G-Hygromycin. Hygromycin-resistant cells were subsequently transduced with pCLIP-Tre3g-hCMV-Cas9P2A-zsGreen. Expression of Cas9-P2A-zsGreen was induced at $1 \mu \mathrm{g}$ $\mathrm{ml}^{-1}$ of doxycycline for $16 \mathrm{~h}$, and zsGreen ${ }^{+}$cells were sorted by flow cytometry. Cells were washed out to remove doxycycline to turn off Cas9 expression (TetOff) and then subsequently transduced with pCLIP-gRNA-hCMV-RFP-gRNA. Sorted $\mathrm{RFP}^{+}$cells were subjected to further experiments. Gene deletion was initiated by treatment with $1 \mu \mathrm{g} \mathrm{ml}^{-1}$ of doxycycline (TetOn). Non-targeting guide RNA was used as control.

\section{Gene deletion by non-viral genome targeting}

Chemically synthesized CRISPR RNAs $(160 \mu \mathrm{M})$ and trans-activating CRISPR RNAs $(160 \mu \mathrm{M})$ were mixed $1: 1$ by volume and annealed by incubation at $37^{\circ} \mathrm{C}$ for $30 \mathrm{~min}$. Recombinantly produced Cas $9(40 \mu \mathrm{M})$ was then mixed 1:1 by volume with gRNA to produce RNA ribonucleoprotein (RNP) complexes. RNPs were freshly complexed before electroporation. Electroporation was performed by using pulse code EH-115 on a Lonza 
4D 96-well electroporation system. Predesigned Alt-R CRISPR-Cas9 guide RNAs were purchased from IDT. Non-targeting control guide RNAs were purchased from IDT.

\section{Co-immunoprecipitation}

Co-immunoprecipitation was performed with the Pierce Crosslink Magnetic IP/Co-IP kit according to manufacturer's instructions (Thermo Fisher Scientific). In brief, patient-derived $\mathrm{Ph}^{+} \mathrm{ALL}$ cells (PDX2) were transduced with MSCV Flag-IRES-Puro or MSCV Flag-IFITM3-IRES-Puro and selected in puromycin for 3 days. Viable cells $\left(5 \times 10^{7}\right)$ were collected and washed by PBS before lysis using IP lysis/Wash buffer (Thermo Fisher Scientific). Then, $5 \mu$ g of anti-Flag antibody M2 (F1804, Sigma-Aldrich) per sample was coupled to protein A/G magnetic beads and covalently crosslinked with $20 \mu \mathrm{M}$ disuccinimidyl suberate. The antibody crosslinked beads were incubated with cell lysate, washed to remove non-bound material and eluted in a low-pH elution buffer that dissociates bound antigen from the antibody crosslinked beads. The enriched antigen in low $\mathrm{pH}$ was immediately neutralized and subjected to western blotting.Jeko1 cells electroporated with either non-targeting RNP or IFITM3-targeting RNP complex were stimulated with $10 \mu \mathrm{g} \mathrm{ml}^{-1}$ $\mathrm{F}\left(\mathrm{ab}^{\prime}\right)_{2}$ fragment goat anti-human $\mu$ chain (Jackson Immunoresearch) for the indicated time points at $37^{\circ} \mathrm{C}$ and $5 \% \mathrm{CO}_{2}$. Cells were immediately washed with chilled PBS and subjected to co-immunoprecipitation with anti-CD19 antibody (no. 90176, CST).

\section{PLA}

For PLA of pre-BCR or BCR-IFITM3, cells were incubated with $10 \mu \mathrm{g}$ $\mathrm{ml}^{-1} \mathrm{~F}\left(\mathrm{ab}^{\prime}\right)_{2}$ fragment goat anti-human $\mu$ chain (Jackson Immunoresearch) for $5 \mathrm{~min}$ at $37^{\circ} \mathrm{C}$ and $5 \% \mathrm{CO}_{2}$. Cells were immediately washed with chilled PBS and subsequently fixed in fixation buffer (Biolegend) containing $4 \%$ paraformaldehyde for $25 \mathrm{~min}$ on ice and then washed with chilled PBS. For cellular membrane staining, cells were labelled with $5 \mu \mathrm{g} \mathrm{ml}^{-1}$ WGA conjugated to Alexa Fluor 488 (Thermo Fisher Scientific) for $5 \mathrm{~min}$ at room temperature. Cells were then permeabilized in Perm/Wash Buffer (BD Biosciences) and then blocked in Duolink Blocking buffer for $30 \mathrm{~min}$ at room temperature. Cells were incubated with 1:150 diluted primary antibodies listed in Supplementary Table 10 overnight at $4{ }^{\circ} \mathrm{C}$. For late endosome staining, cells were incubated with anti-human LAMP1 conjugated to Alexa Fluor 488 (R\&D Systems) with the primary antibodies. Cells were washed and settled on Cell-Tak (Corning)-coated Shandon Single Cytoslide by cytospin at $400 \mathrm{~g}$ for 5 min. PLA reactions were carried out according to the manufacturer's protocol (Duolink, Sigma). In brief, primary antibodies were coupled with Duolink in situ PLA PLUS or MINUS probes (Sigma-Aldrich), and then probes were visualized with Duolink Detection Reagent Red (Sigma-Aldrich). Cells were mounted with Duolink in situ mounting medium with DAPI (Sigma-Aldrich). Microscopy images were acquired using an Olympus IX3-55 and analysed by CellSens imaging software (Olympus) and ImageJ. For quantification of PLA signals, one dot was defined as pixel size of $5 \times 5$ by BlobFinder software. Statistical significance was calculated using unpaired Student's $t$-test with Excel and plotted with GraphPad Prism 7.

\section{Phosphoproteomic analysis}

Patient-derived B-ALL (PDX2) cells transduced with HA-tagged IFITM3(Y2OE) or empty vector control were incubated with $2.5 \mu \mathrm{g} \mathrm{ml}^{-1}$ of polyclonal anti-HA (Abcam) for $5 \mathrm{~min}$ at $37{ }^{\circ} \mathrm{C}$ at $5 \% \mathrm{CO}_{2}$. Cellular extracts were prepared in urea lysis buffer, sonicated, centrifuged, reduced with DTT and alkylated with iodoacetamide. Fifteen $\mathrm{mg}$ total protein for each sample was digested with trypsin, and $500 \mu \mathrm{g}$ total protein for each sample was digested with LysC and trypsin mix for IMAC analysis. Samples were purified over $\mathrm{C} 18$ columns and dried in a lyophilizer. Dried samples were resuspended and enriched with Fe-IMAC beads, purified over C18STAGE tips (Rappsilber). Replicate injections of each sample were run nonsequentially on the instrument. Peptides were eluted using a 150 - $\min$ (IMAC) linear gradient of acetonitrile in $0.125 \%$ formic acid delivered at $280 \mathrm{nl} / \mathrm{min}$. Tandem mass spectra (MS/MS) were collected in a data-dependent manner with a Thermo Orbitrap Fusion Lumos Tribrid mass spectrometer using a top-twenty MS/MS method, a dynamic repeat count of one and a repeat duration of $30 \mathrm{~s}$. Real-time recalibration of mass error was performed using lock mass (Olsen) with a singly charged polysiloxane ion of $m / z=371.101237$.MS/ MS spectra were evaluated using SEQUEST and the Core platform from Harvard University (by J. K. Eng, E. L. Huttlin and J. Villen). Files were searched against the SwissProt Homo sapiens FASTA database. A mass accuracy of \pm 5 ppm was used for precursor ions and 0.02 Da for product ions. Enzyme specificity was limited to trypsin, with at least one tryptic (K-or R-containing) terminus required per peptide and up to four miscleavages allowed. Cysteine carboxamidomethylation was specified as a static modification, and oxidation of methionine and phosphorylation on serine, threonine and tyrosine residues were allowed as variable modifications. Reverse decoy databases were included for all searches to estimate false discovery rates, and filtered using a $1 \%$ false-discovery rate in the linear discriminant module of Core. Peptides were also manually filtered using a $\pm 5 \mathrm{ppm}$ mass error range and presence of a phosphorylated residue. All quantitative results were generated using Skyline (MacLean) to extract the integrated peak area of the corresponding peptide assignments. Accuracy of quantitative data was ensured by manual review in Skyline or in the ion chromatogram files.

\section{Bio-ID proteomics}

Patient-derived PDX2 B-ALL cells and Jeko1 MCL cells were transduced with Flag-tagged IFITM3(Y2OE) or IFITM3(K83A/K104A) mutant constructs with $\mathrm{N}$-terminal Turbo-ID BirA (engineered biotin ligase) and selected by puromycin for three days. Turbo-ID BirA-expressing construct tagged with Flag was used as negative control. To induce biotinylation of proteins proximal to IFITM3, cells were treated with 50 $\mu \mathrm{moll}^{-1}$ biotin and with anti-HA antibody for simultaneous induction of IFITM 3 crosslinking for 10 min. Cells were washed three times with chilled PBS and lysed in IP/WASH buffer (Thermo Fisher Scientific) with $1 \times$ HALT protease inhibitor (Thermo Fisher Scientific). The lysates were incubated by streptavidin C1 MyOne Dynabeads (Invitrogen) for $16 \mathrm{~h}$ at $4{ }^{\circ} \mathrm{C}$. Unbound proteins were washed 3 times by $2 \%$ SDS-PBS, 3 times by PBS and 3 times with pure water. The eluted proteins were gel-purified, followed by in-gel digestion and subjected to mass spectrometry. For liquid chromatography (LC)-MS/MS analysis, peptides were analysed using a Dionex UltiMate 3000 Rapid Separation LC system and a Orbitrap mass spectrometer (ThermoFisher Scientific). Six- $\mu$ l peptide samples were loaded onto the trap column, which was $150 \mu \mathrm{m} \times 3 \mathrm{~cm}$ and in-house-packed with $3-\mu \mathrm{m} \mathrm{C18}$ beads. The analytical column was a $75 \mu \mathrm{m} \times 10.5-\mathrm{cm}$ PicoChip column packed with $3-\mu \mathrm{m} \mathrm{C18}$ beads (New Objectives). The flow rate was kept at $300 \mathrm{nl} / \mathrm{min}$. Solvent A was $0.1 \%$ formic acid (FA) in water and solvent $B$ was $0.1 \%$ FA in ACN. The peptide was separated on a 120-min analytical gradient from $5 \%$ ACN and $0.1 \%$ FA to $40 \% \mathrm{ACN}$ and $0.1 \%$ FA. The mass spectrometer was operated in data-dependent mode. The source voltage was $2.40 \mathrm{kV}$ and the capillary temperature was $275^{\circ} \mathrm{C}$. MS1 scans were acquired from $400-2,000 \mathrm{~m} / z$ at 60,000 resolving power and automatic gain control (AGC) set to $1 \times 10^{6}$. The 15 most abundant precursor ions in each MS1 scan were selected for fragmentation. Precursors were selected with an isolation width of $1 \mathrm{Da}$ and fragmented by collision-induced dissociation at $35 \%$ normalized collision energy in the ion trap; previously selected ions were dynamically excluded from reselection for $60 \mathrm{~s}$. The MS2 AGC was set to $3 \times 10^{5}$. For data analysis, proteins were identified from the mass spectrometry raw files using Mascot search engine (Matrix science). MS/MS spectra were searched against the SwissProt human database. All searches included carbamidomethyl cysteine as a fixed modification and oxidized Met, deamidated Asn and GIn, and acetylated $\mathrm{N}$ terminus as variable modifications. Three missed tryptic cleavages were allowed. The MS1 precursor mass tolerance was set to 
$10 \mathrm{ppm}$ and the MS2 tolerance was set to $0.6 \mathrm{Da}$. A $1 \%$ false discovery rate cut-off was applied at the peptide level. Only proteins with a minimum of two peptides above the cut-off were considered for further study. For comparison to empty vector control, background peptide abundances for missing values were imputed from a Gaussian distribution centred around the minimum observed abundance using the MinProb method from MSnbase package in $\mathrm{R}$.

\section{Cell-surface proteome analyses}

Cell-surface proteins were labelled with biotin using the $\mathrm{N}$-linked glycosylation-site biotin labelling method ${ }^{39}$. In brief, 40 million fitm $^{+/+}$ or Ifitm $3^{-/-}$B-ALL cells were washed twice and resuspended in $1 \mathrm{ml}$ of ice-cold PBS, and treated with $1.6 \mathrm{mM}$ sodium metaperiodate (VWR) at $4{ }^{\circ} \mathrm{C}$ for $20 \mathrm{~min}$ to oxidize the vicinal diols of sugar residues linked to surface proteins. The cells were then washed twice in PBS to remove excess sodium metaperiodate. Cells were resuspended in $1 \mathrm{ml}$ of ice-cold PBS and treated with $1 \mathrm{mM}$ biocytin hydrazide (Biotium) and $10 \mathrm{mM}$ aniline (Sigma-Aldrich) at $4{ }^{\circ} \mathrm{C}$ for $90 \mathrm{~min}$ with gentle mixing to biotinylate free aldehydes exposed on the sugar residues. After labelling, cells were washed 3 times with ice-cold PBS to removed excess biotin, frozen in liquid nitrogen and stored at $-80^{\circ} \mathrm{C}$ until further processing for mass spectrometry. All experiments were performed in biological triplicates with replicates collected from consecutive passages. Frozen cell pellets were thawed on ice in $1 \mathrm{ml}$ of RIPA buffer (Millipore) with the addition of $1 \times$ HALT protease inhibitors (Pierce). After incubation on ice for $10 \mathrm{~min}$, cells were disrupted by sonication and the lysates were clarified by centrifugation at $17,000 \mathrm{rcf}$ at $4{ }^{\circ} \mathrm{C}$ for $10 \mathrm{~min}$. Clarified lysate was mixed with $500 \mu \mathrm{l}$ of neutravidin agarose resin (Thermo Fisher Scientific) and incubated at $4{ }^{\circ} \mathrm{C}$ for $2 \mathrm{~h}$ with end-over-end mixing. Neutravidin beads with captured biotinylated surface proteins were washed extensively by gravity flow to remove unbound proteins using $50 \mathrm{ml}$ of $1 \times$ RIPA $+1 \mathrm{mM}$ EDTA, followed by $50 \mathrm{ml}$ of PBS $+1 \mathrm{M} \mathrm{NaCl}$ and finally $50 \mathrm{ml}$ of $50 \mathrm{mM} \mathrm{ABC}+2 \mathrm{M}$ urea buffer. Washed beads were resuspended in digestion buffer ( $50 \mathrm{mM}$ Tris pH 8.5,10 mM TCEP, $20 \mathrm{mM}$ 2 -iodoacetamide and $1.6 \mathrm{MUrea}$ ) with $10 \mu \mathrm{g}$ of added trypsin protease (Pierce, 90057) to perform simultaneous disulfide reduction, alkylation and on-bead peptide digestion at room temperature overnight (16-20 h). After digestion, the pH was dropped to about 2 with neat trifluoroacetic acid (Sigma-Aldrich) and the peptide mixture was desalted using a SOLA-HRP column (Thermo Fisher Scientific) on a vacuum manifold. Desalted peptides were eluted with $50 \%$ acetonitrile (Sigma-Aldrich) and 50\% water with 0.1\% TFA and dried down completely in a speedvac. Dried peptides were resuspended in LCMS grade water (Fisher) with $2 \% \mathrm{ACN}$ and $0.1 \%$ FA. Peptide concentration was measured using $280 \mathrm{~nm}$ absorbance on a Nanodrop, and the peptide concentration was adjusted to $0.2 \mu \mathrm{g} \mathrm{\mu l}^{-1}$ for mass spectrometry runs.

\section{LC-MS and data analysis for cell-surface proteome}

For each replicate, $1 \mu \mathrm{g}$ of peptide was injected onto a Dionex Ultimate 3000 NanoRSLC instrument with a 15-cm Acclaim PEPMAP C18 (Thermo Fisher Scientific, 164534) reverse-phase column. The samples were separated on a 3.5-h nonlinear gradient using a mixture of buffer $\mathrm{A}(0.1 \%$ $\mathrm{FA})$ and $\mathrm{B}(80 \% \mathrm{ACN} / 0.1 \% \mathrm{FA})$, from $2.4 \% \mathrm{ACN}$ to $32 \% \mathrm{ACN}$. Eluted peptides were analysed with a Thermo $Q$-Exactive Plus mass spectrometer. The mass spectrometry survey scan was performed over a mass range of $350-1500 \mathrm{~m} / \mathrm{z}$ with a resolution of 70,000 , with a maximum injection time of $100 \mathrm{~ms}$. We performed a data-dependent MS2 acquisition at a resolution of $17,500, \mathrm{AGC}$ of $5 \times 10^{4}$, and injection time of $150 \mathrm{~ms}$. The 15 most intense precursor ions were fragmented in the higher-energy collisional dissociation at a normalized collision energy of 27. Dynamic exclusion was set to 20 s to avoid over-sampling of highly abundant species. The raw spectral data files are available at the ProteomeXchange PRIDE repository (accession number PXD014691). Raw spectral data were analysed using MaxQuant v.1.5.1.2 ${ }^{40}$ to identify and quantify peptide abundance and searched against the human Swiss-Prot annotated human proteome from Uniprot (downloaded with 20,303 entries). The 'match-between-runs' option was selected to increase peptide identifications and the 'fast LFQ' option was selected to calculate label-free quantification values of identified proteins. All other settings were left at the default MaxQuant values. The MaxQuant output data were analysed using Perseus ${ }^{41}$ and the R program (version 3.4.0). Proteins annotated as 'reverse', 'only identified by site' and 'potential contaminant' were filtered out, as were proteins that were quantified in less than two out of three biological replicates in at least one experimental group. Missing values were imputed on the basis of the normal distribution of the dataset as implemented by Perseus. Volcano plots were generated using output from a two-sample $t$-test comparing the $\log _{2}$-transformed label-free quantification protein abundance values from different cell lines with a false-discovery rate set to 0.01 . Validation was performed by flow cytometry (Supplementary Table 1).

\section{Cholesterol and lipid raft measurement}

For the depletion of cholesterol, cells were preincubated with $5 \mathrm{mM}$ of methy- $\beta$-cyclodextrin for $30 \mathrm{~min}$ at $37^{\circ} \mathrm{C}$ before filipin staining. For ganglioside GM1 staining, cells were labelled with cholera toxin $B$ using Vybrant lipid raft labelling kit (Molecular Probes), according to the manufacturer's protocol. In brief, cells were labelled with cholera toxin unit B conjugated with Alexa Fluor 594 on ice for $15 \mathrm{~min}$, washed twice with PBS. Cholera-toxin-B-labelled lipid rafts were crosslinked with anti-cholera-toxin-B antibody on ice for $15 \mathrm{~min}$, washed twice with PBS and analysed by flow cytometry.

\section{In vivo transplantation of leukaemia cells}

Mouse pre-B ALL cells transformed by BCR-ABL1 or NRAS(G12D) were injected into sublethally irradiated (200 cGy) NOD-scid IL2Rg ${ }^{\text {null }}$ (NSG) mice via the tail vein. Eight- to ten-week-old female NSG mice were randomly allocated before injection. A mouse was euthanized when they showed signs of leukaemia burden, such as a hunched back, weight loss and inability to move, and then the bone marrow and/or spleen were collected to test leukaemia infiltration by flow cytometry. Kaplan-Meier survival analysis was performed using GraphPad Prism 7 (GraphPad Software) to compare overall survival. The Mantel-Cox log-rank test was used as statistical analysis using GraphPad Prism 7 (Supplementary Table 3). The minimal number of mice in each group was calculated by using the 'cpower' function in Hmisc package of R. No blinding was used. All mouse experiments were subject to institutional approval by the Beckman Research Institute of City of Hope Animal Care and Use Committee.

\section{PIP3 quantification}

For Fig. 4, 60 million viable cells were resuspended in chilled $0.5 \mathrm{M}$ trichloroacetic acid (TCA) in a total volume of $1 \mathrm{ml}$ and incubated on ice for $5 \mathrm{~min}$. Cells were centrifuged at 3,000 rpm for $7 \mathrm{~min}$ at $4{ }^{\circ} \mathrm{C}$ and resuspended in 5\% TCA and $1 \mathrm{mM}$ EDTA in a total volume of $1 \mathrm{ml}$. After vortexing for $30 \mathrm{~s}$, cells were washed again with $5 \%$ TCA and $1 \mathrm{mMEDTA}$. To extract neutral lipids, cells were resuspended in $1 \mathrm{ml}$ of $\mathrm{MeOH}: \mathrm{CHCl}_{3}$ (2:1) and vortexed for $10 \mathrm{~min}$ at room temperature and centrifuged at 3,000 rpm for $5 \mathrm{~min}$. After 1 more extraction of neutral lipids, the acidic lipids were extracted by adding $750 \mu \mathrm{l} \mathrm{MeOH}: \mathrm{CHCl}_{3}: 12 \mathrm{M} \mathrm{HCl}$ (80:40:1) with vigorous vortexing for $25 \mathrm{~min}$ at room temperature. After centrifugation at 3,000 rpm for $5 \mathrm{~min}$, supernatant was transferred to a 2-ml centrifuge tube and mixed with $250 \mu \mathrm{l} \mathrm{of} \mathrm{CHCl}_{3}$ and $450 \mu \mathrm{l}$ of $0.1 \mathrm{M} \mathrm{HCl}$, vortexed for $30 \mathrm{~s}$, and centrifuged at 3,000 rpm for $5 \mathrm{~min}$ to separate organic and aqueous phases. Five hundred $\mu \mathrm{l}$ of the lower organic phases was collected into a clean $1.5-\mathrm{ml}$ vial and dried in a vacuum dryer for $1 \mathrm{~h}$. Dried lipid was stored at $-20^{\circ} \mathrm{C}$. On the day of assay, lipid samples were reconstituted with $200 \mu$ l of PBS containing $0.25 \%$ protein stabilizer; $20 \mu \mathrm{l}$ was used for $\mathrm{PI}(4,5) \mathrm{P}_{2}$ measurement and 
the rest $(180 \mu \mathrm{l})$ was used for $\mathrm{PI}(3,4,5) \mathrm{P}_{3}$ measurement using an ELISA kit (Echelon Biosciences) according to the manufacturer's instructions.

\section{Exogenous delivery of PIP3}

Phosphatidylinositol3,4,5-trisphosphate diC16 (Echelon Biosciences) was freshly reconstituted with PBS at $2 \mathrm{mM}$. Unlabelled shuttle PIP carrier 2 (histone $\mathrm{H1}$ ) was freshly reconstituted with water at $2 \mathrm{mM} . \mathrm{PIP}_{3}$ in a volume of $75 \mu \mathrm{l}$ was mixed with $25 \mu \mathrm{l}$ of PIP carrier and incubated at room temperature for $10 \mathrm{~min}$. One hundred $\mu \mathrm{l}$ of $\mathrm{PIP}_{3}$-carrier complex was added into Ifitm $3^{+/+}$or Ifitm $3^{-/-}$B-ALL cells in a total volume of $5 \mathrm{ml}$ and incubated for $24 \mathrm{~h}$. $\mathrm{PIP}_{3}$-loaded $/$ fitm $^{+/ /+}$or $/ \mathrm{fitm}^{-/-}$B-ALL cells were subjected to colony-forming assay, as shown in Extended Data Fig. 8.

\section{Lipid-binding assay}

To assess the direct binding of IFITM3 to lipids, a lipid-binding assay was performed using Membrane Lipid Strips (Echelon Biosciences) according to the manufacturer's instructions. In brief, membranes were blocked in 5\% fatty acid-free BSA (Sigma-Aldrich) in TBST ( $50 \mathrm{mM}$ Tris- $\mathrm{HCl}, 150 \mathrm{mM} \mathrm{NaCl}$, and $0.1 \%$ Tween 20 ) for $1 \mathrm{~h}$ at room temperature in the dark followed by overnight incubation with $0.5 \mu \mathrm{g} / \mathrm{ml}$ of recombinant proteins in blocking buffer at $4^{\circ} \mathrm{C}$ with gentle agitation. After washing membranes 3 times for 30 min in TBST, membranes were incubated for $1 \mathrm{~h}$ with anti-GST tag polyclonal $\mathrm{Ab}$ (Thermo Fisher Scientific) or anti-biotin monoclonal antibody (Cell Signaling Technology) antibodies, listed in Supplementary Table 10. Membranes were incubated with alkaline-phosphatase-conjugated secondary antibodies (Invitrogen) and chemiluminescent substrate (Invitrogen) and were further detected by UVP BioSpectrum 810 Imaging System (Thermo Fisher Scientific). Recombinant GST-tagged protein was purchased from Sigma-Aldrich. Recombinant human GST-IFITM3 protein was purchased from Abnova. Recombinant human IFITM3 fragments listed in Supplementary Table 5 were synthesized at LifeTein.

\section{Preferential PIP3 accumulation and binding to IFITM3 using multi-scale molecular dynamics simulations}

The IFITM 3 protein structure from residues 58 to 128 was modelled by coarse-grained molecular dynamics simulation method in GROMACS, in explicit cell membrane mimic bilayer. There is no structure was available for IFITM3 or for any close homologue. We calculated the hydrophobicity for each position using the hydrophobic index of amino acids ${ }^{42}$ and averaged hydrophobicity over a sliding window of seven amino acids. Previous structural studies on IFITM3 structure ${ }^{43}$ have shown there is only one transmembrane domain 2 that is inserted into the membrane. On the basis of this evidence, we used the topology shown in Supplementary Fig. 9 as the starting structure. TM1 was generated as an $\alpha$-helix structure, and the CRAC motifs and linker region were built as fully extended chain. The linker region was relaxed using 5,000 steps of coarse-grained simulation using GROMACS program ${ }^{44}$ with Martini force field ${ }^{45}$ to remove stress in the system. We then added the $\mathrm{TM} 2$ region as a helix and inserted TM 2 into a cell-membrane-mimicking bilayer $^{46}$. The composition and ratio of lipids for the mixed bilayer is POP C:DOPC:POPE:DOPE:CHOL:Sph:GM1 = 0.2:0.2:0.05:0.05:0.25:0.15:0.1 for the outer leaflet of the bilayer, and 0.05:0.05:0.20:0.2:0.08:0.0 8:0.25:0.03:0.03:0.03 of POPC: DOPC: POPE: DOPE: POPS: DOPS: CHOL:PIP1:PIP2:PIP3 for the inner leaflet. We generated three possible starting setup for the mixed lipid bilayer with water in the upper and inner regions with CHARMM-GUI ${ }^{47,48}$. The charges in the system were neutralized with $0.15 \mathrm{M} \mathrm{NaCl}$. We used nine replicates of the protein IFITM3 structure in the simulation box (Supplementary Fig. 9a). The initial configuration was minimized using steepest decent method for 5,000 steps, and then equilibrated in NPT ensemble for $5 \mathrm{~ns}$, at 303-K temperature and 1 bar pressure. The temperature was controlled by Berendsen thermostat ${ }^{49}$ with coupling constant of $1 \mathrm{ps}$, and the pressure was controlled by Berendsen barostat ${ }^{49}$ in semi-isotropic type with a coupling constant of $5 \mathrm{ps}$ and a compressibility of $3 \times 10^{-4}$ bar $^{-1}$.
The reaction field coulomb ${ }^{50}$ was applied to describe electrostatics, with cut-off at $1.1 \mathrm{~nm}$. The cut-off for van der Waals interactions used was also $1.1 \mathrm{~nm}$. We used the Leap-frog integrator, and a 20-fs time step for integration. All systems were solvated in coarse-grained water model adopted in the Martini forcefield water, neutralized with $0.15 \mathrm{M} \mathrm{NaCl}$. Ten $\mu$ s of production simulation was performed for each of the three starting conformations of the lipid bilayer. The final $1 \mu \mathrm{s}$ of the trajectory from each of the 3 setups was used to analyse the folded structure of the CRAC1-basic patch-CRAC2 region of the IFITM3 model. We clustered the conformations of IFITM3 by the root mean square deviation cut-off of $0.3 \mathrm{~nm}$ in the backbone coarse-grained particles in the coarse-grained simulations. We analysed the binding patterns of PIP2 and PIP3 in this most populated conformational cluster. We observed that both PIP2 and PIP3 compete for the 'basic patch' of residues shown in the amino acid sequence in Extended Data Fig. 9. We extracted three snapshots from this conformational cluster that represent the most diverse patterns in PIP2 and PIP3 binding.

\section{Details of the all-atom molecular dynamics simulations for phospholipid binding}

To better understand the detailed binding conformation of PIP2 and PIP3 to IFITM3 and to calculate their binding energies to IFITM3, we converted the three snapshots extracted from the coarse-grained simulations to an all-atom system using Martini tools ${ }^{51}$. The three chosen snapshots were cut into $9 \times 9-\mathrm{nm}^{2}$ box centred at the IFITM3 unit from the coarse-grained simulations to preserve the local optimized lipid environment. The lipid and protein were converted to an all-atom model, and were resolvated into a $9 \times 9 \times 9-\mathrm{nm}^{3}$ simulation box, neutralized by $0.15 \mathrm{M} \mathrm{NaCl}$. Extended Data Figure 9 shows the detailed interaction between PIP2 or PIP3 with IFITM3 protein in an all-atom resolution base on these snapshots. The all-atom simulations were performed using GROMACS package ${ }^{44}$ and CHARMM 36 force field ${ }^{52}$, with the TIP3 water model. The nonbond interactions were calculated with a cut-off of $12 \AA$, and the particle-mesh Ewald method ${ }^{51}$ was applied to solve long-range van der Waals interactions. Each system was gradually heated to $310 \mathrm{~K}$ with random initial velocities sampled from a Boltzmann distribution. The heating process lasted for 1ns, with temperature controlled by a Nosé-Hoover thermostat ${ }^{53}$, followed by 30 -ns equilibration in NPT ensemble with harmonic position restraints on protein heavy atoms. The pressure was maintained at 1 bar in semi-isotropic environment, controlled by the Parrinello-Rahman method. The restraint force was gradually reduced from $5 \mathrm{kcal} /$ $\mathrm{mol}$ to $0 \mathrm{kcal} / \mathrm{mol}$ with a $-1 \mathrm{kcal} / \mathrm{mol} \mathrm{step} \mathrm{per} 5$-ns window. The final frame of equilibration was taken for a production run. The production run was performed twice for $50 \mathrm{~ns}$ each, with 2 different random initial velocities assigned to the equilibrated structure. An integration time step of $2 \mathrm{fs}$ was used. The interaction energy between PIP3 with IFITM3 protein was calculated as the sum of electrostatic Coulombic energy and van der Waals potential energy averaged over the two 50-ns trajectories, totalling $100 \mathrm{~ns}$.

\section{Interaction of PIP3 with the two basic residue patches}

The PIP3-binding and AKT-signalling activation assays showed that mutation of the K83/K104 patch to K83A/K104A had higher effect than mutating the $\mathrm{R} 85 / \mathrm{R} 87 / \mathrm{K} 88$ patch to $\mathrm{R} 85 \mathrm{~A} / \mathrm{R} 87 \mathrm{~A} / \mathrm{K} 88 \mathrm{~A}$. To further validate the interaction strength difference between PIP3 and the two basic patches, we converted all PIP1 and PIP2 in the simulation box to PIP3. We further performed simulations on the IFITM3(K83A/K104A) and on IFITM3(R85A/R87A/K88A) in the PIP3-only simulation box. Starting from this structure, we performed equilibration of the system and 5 all-atom molecular dynamics production simulations (each 200-ns long), using the protocol for all-atom molecular dynamics simulations described in 'Details of the all-atom molecular dynamics simulations for phospholipid binding'. We aggregated the final $100 \mathrm{~ns}$ of simulation trajectories from each of the 5 runs, which we added to 500 ns of 
molecular dynamics simulation trajectories for analysis. We calculated the interaction energy as the sum of electrostatic Coulombic and van der Waals potential energies between PIP3 and residues forming the basic patch only (K83/K104 or R85/R87/K88). The interaction energies were averaged over the 500 ns of aggregated molecular dynamics simulation trajectories for each system. We repeated these interaction energy calculations for the wild-type IFITM3 and for the mutant $\mathrm{K} 83 \mathrm{~A} / \mathrm{K} 104 \mathrm{~A}$ and R85A/R87A/K88A constructs.

\section{Contact-frequency heat-map calculations from molecular dynamics simulations}

The important information that can be extracted from molecular dynamics simulations is the temporal frequency of PIP3 contacts with the two basic residue patches. The persistence of these interactions has an important role in the accumulation of PIP3 by IFITM3. We calculated the percentage of molecular dynamics snapshots that show contacts between each of the residues in the basic patch. This is termed the contact frequency. We also calculated the percentage of molecular dynamics snapshots that show simultaneous contacts made by PIP3 with two or more residues in the two basic residue patches. We generated a heat map using the frequencies calculated for the wild-type IFITM 3 and in the alanine mutants.

\section{Quantitative real-time PCR}

Bone marrow from a healthy donor was stained and sorted as previously described $^{54}$. Total RNA from cells was extracted using the RNA isolation kit from Macherey-Nagel. Complementary DNA was generated with the qScript cDNA SuperMix (Quanta Biosciences). Quantitative real-time PCR was performed with FAST SYBR Green Master Mix (Applied Biosystems) and the Vii7 real-time PCR system (Applied Biosystems) according to standard PCR conditions. $C O X 6 B$ was used as a reference gene.

\section{Acoustic scattering measurements}

Single-cell size-normalized acoustic scattering (SNACS) was measured using a previously established microfluidic method, which has been shown to be specifically sensitive to cell-surface stiffness ${ }^{55}$. Full measurement details can be found in a previous publication ${ }^{55}$. In brief, cells were flowed through a standing acoustic wave generated inside a vibrating suspended microchannel resonator (SMR). The SMR is a cantilever-based mass measurement tool ${ }^{56}$, which can also detect acoustic scattering from cells when the cells transit through the acoustic wave. The cantilever vibration frequency was monitored, and its shift was used to quantify the acoustic scattering from the cells as well as the buoyant mass of the cells. Before taking a set of measurements, the SMR was cleaned with $0.25 \%$ trypsin-EDTA for $20 \mathrm{~min}$, followed by $5 \%$ bleach for 3-min and then a 5-min rinse with deionized $\mathrm{H}_{2} \mathrm{O}$, to remove persistent biological debris. After cleaning, the SMR was passivated with $1 \mathrm{mg} / \mathrm{ml} \mathrm{PLL-g-PEG} \mathrm{in} \mathrm{H}_{2} \mathrm{O}$ for 10 min at room temperature, followed by a 5 -min rinse with normal cell culture medium. During the measurement, all the samples were loaded into the SMR through 0.005-inch-inner-diameter fluorinated ethylene propylene tubing. The fluid flow across the SMR was driven by three independent electronic pressure regulators and three solenoid valves. A consistent differential pressure was applied across the SMR to maintain constant shear and data rate for cell measurement. The data displayed in Extended Data Fig. 8a were obtained using a $350-\mu \mathrm{m}$-long cantilever with a $15 \times 20-\mu \mathrm{m}$-sized channel inside of the cantilever and an approximately 200-ms transit time through the cantilever. All the regulators, valves and data acquisition were controlled by custom software coded in LabVIEW 2017 (National Instruments) as detailed in a previous publication $^{55}$. A parallel volume measurement using Coulter Counter (Beckman Coulter) was carried out to quantify average cell volume, which was used together with the single-cell buoyant mass measurements to calculate SNACS for each cell, as previously reported ${ }^{55}$. All measurements were carried out in normal cell culture medium at room temperature within 10 min of taking cells out of the cell culture incubator. After a measurement of the untreated Jekol cells, a new patch of same cells was obtained from the incubator, treated with $10 \mu \mathrm{g} \mathrm{ml}^{-1} \mathrm{~F}\left(\mathrm{ab}^{\prime}\right)_{2}$ fragment goat anti-human $\mu$ chain (Jackson Immunoresearch) and the cells were immediately (within about $1 \mathrm{~min}$ ) loaded into the SMR for measurement. Antibody-treated Jeko1 cells were measured for about 7 min to ensure that the anti-human $\mu$ chain stimulated changes were not reversed during the experiment. The SMR was briefly washed with PBS between each experiment. Wild-type Jeko1 cells were also measured after fixation to obtain a positive control for cell stiffness. For fixation, the cells were washed twice with PBS, mixed with 8\% PFA for $30 \mathrm{~min}$, washed twice with PBS and stored at $4{ }^{\circ} \mathrm{C}$ before the acoustic scattering measurements.

\section{Quantification and statistical analysis}

Data are shown as mean \pm s.d. unless stated. Statistical analysis was performed by GraphPad Prism 7 (GraphPad Software) using unpaired two-tailed $t$ test, or as indicated in figure legends. Significance was considered at $P<0.05$. For in vivo transplantation experiments, the minimal number of mice in each group was calculated through use of the 'cpower' function in the Hmisc package of R. Kaplan-Meier survival analysis was used to estimate overall survival with GraphPad Prism 7. Mantel-Cox log-rank test was used to compare the difference between two groups. No mice were excluded. For patient overall survival analysis, patients in each dataset were divided into two groups on the basis of whether their expression was above or below the median level of IFITM3 and Kaplan-Meier survival analysis was used to estimate overall survival. The datasets used include patient-outcome data for B-ALL (Children's Oncology Group (COG) P9906, $n=207$; Eastern Cooperative Oncology Group (ECOG) E2993, $n=83$; and St Jude, $n=15$ ), mantle cell lymphoma (Lymphoma/Leukemia Molecular Profiling Project (LLMPP), $n=92$ ) and acute myeloid leukaemia (The Cancer Genome Atlas (TCGA), $n=200$ ). A log-rank test was used to compare survival differences between patient groups. The R package 'survival' (version 2.35-8) was used for the survival analysis and Cox proportional hazards regression model in the R package for the multivariate analysis (https:// www.r-project.org/). The investigators were not blinded to allocation during experiments and outcome assessment. Experiments were repeated to ensure reproducibility of the observations.

\section{Reporting summary}

Further information on research design is available in the Nature Research Reporting Summary linked to this paper.

\section{Data availability}

Patient-outcome data for B-ALL were obtained from the National Cancer Institute TARGET DATA Matrix of the COG Clinical Trial P9906 (GSE11877) (refs. ${ }^{28,30}$ ), ECOG Clinical Trial E2993 (GSE5314) $\left(\right.$ ref. $^{57}$ ) and St Jude Children's Research Hospital (https://www.stjuderesearch. org/site/data/ALL3/) (ref. ${ }^{29}$ ). Patient-outcome data for mantle cell lymphoma were obtained from https://llmpp.nih.gov/MCL/ (ref. ${ }^{58}$ ). Patient-outcome data for AML were obtained from TCGA Acute Myeloid Leukaemia Project (http://www.cbioportal.org/study/ summary?id=laml_tcga_pub\#clinical $)^{59}$. Proteomics data have been deposited to the ProteomeXchange Consortium via the PRIDE partner repository with the following accession numbers: cell-surface proteome, PXD014691; phosphoproteome, PXD020696; and IFITM3 interactomes, PXD020697. Levels of IFITM3 mRNA across human normal and malignant B-lymphoid samples were obtained from http:// Amazonia.transcriptome.eu/. All other data are available from the corresponding author upon reasonable request. Genome binding and occupancy profiling from wild-type and IKDN stromal adherent pre-B cells were obtained from GSE86897. Immunohistochemistry images for IFITM3 levels in normal or malignant B cells were obtained from 
The Human Protein Atlas https://www.proteinatlas.org/. ChIP-seq data of the genome-wide mapping of IKZF1 binding in patient-derived B-ALL xenograft cells were obtained from GSE58825. ChIP-seq data of the genetic analysis of IKZF1 target genes and tumour suppressor function in $\mathrm{Ph}^{+}$pre-B ALL cells were obtained from GSE90656. RNA-sequencing data for Ifitm $3^{+/+}$and $I$ fitm $3^{-/-} B C R-A B L 1$ or $N R A S^{G 12 D} \mathrm{~B}$-ALL cells are available at GSE155305. RNA-sequencing data for $P$ ten ${ }^{f l / f l}$ pre-B cells carrying 4-OHT-inducible Cre-ER ${ }^{\mathrm{T} 2}$ or $\mathrm{ER}^{\mathrm{T} 2}$ are available at GSE155618. Supplementary Table 12 summarizes the accession numbers and publicly deposited data from this study. All other data needed to evaluate the conclusions in the paper are available within the Article and its Supplementary Information. Source data are provided with this paper.

27. Geng, H. et al. Integrative epigenomic analysis identifies biomarkers and therapeutic targets in adult B-acute lymphoblastic leukemia. Cancer Discov. 2, 1004-1023 (2012).

28. Harvey, R. C. et al. Identification of novel cluster groups in pediatric high-risk B-precursor acute lymphoblastic leukemia with gene expression profiling: correlation with genomewide DNA copy number alterations, clinical characteristics, and outcome. Blood 116, 4874-4884 (2010).

29. Ross, M. E. et al. Classification of pediatric acute lymphoblastic leukemia by gene expression profiling. Blood 102, 2951-2959 (2003)

30. Kang, H. et al. Gene expression classifiers for relapse-free survival and minimal residual disease improve risk classification and outcome prediction in pediatric B-precursor acute lymphoblastic leukemia. Blood 115, 1394-1405 (2010).

31. Borowitz, M. J. et al. Clinical significance of minimal residual disease in childhood acute lymphoblastic leukemia and its relationship to other prognostic factors: a Children's Oncology Group study. Blood 111, 5477-5485 (2008).

32. Poddar, S., Hyde, J. L., Gorman, M. J., Farzan, M. \& Diamond, M. S. The interferon-stimulated gene IFITM3 restricts infection and pathogenesis of arthritogenic and encephalitic alphaviruses. J. Virol. 90, 8780-8794 (2016).

33. Foley, S. B. et al. Expression of BCR/ABL p210 from a knockin allele enhances bone marrow engraftment without inducing neoplasia. Cell Rep. 5, 51-60 (2013).

34. Schjerven, $\mathrm{H}$. et al. Genetic analysis of Ikaros target genes and tumor suppressor function in BCR-ABL1+ pre-B ALL. J. Exp. Med. 214, 793-814 (2017).

35. Dobin, A. et al. STAR: ultrafast universal RNA-seq aligner. Bioinformatics 29, 15-21 (2013).

36. Liao, Y., Smyth, G. K. \& Shi, W. featureCounts: an efficient general purpose program for assigning sequence reads to genomic features. Bioinformatics 30, 923-930 (2014).

37. Robinson, M. D., McCarthy, D. J. \& Smyth, G. K. edgeR: a Bioconductor package for differential expression analysis of digital gene expression data. Bioinformatics $\mathbf{2 6}$, 139-140 (2010).

38. Yu, G., Wang, L.-G. \& He, Q.-Y. ChIPseeker: an R/Bioconductor package for ChIP peak annotation, comparison and visualization. Bioinformatics 31, 2382-2383 (2015)

39. Wollscheid, B. et al. Mass-spectrometric identification and relative quantification of $\mathrm{N}$-linked cell surface glycoproteins. Nat. Biotechnol. 27, 378-386 (2009).

40. Tyanova, S., Temu, T. \& Cox, J. The MaxQuant computational platform for mass spectrometry-based shotgun proteomics. Nat. Protocols 11, 2301-2319 (2016).

41. Tyanova, S. et al. The Perseus computational platform for comprehensive analysis of (prote)omics data. Nat. Methods 13, 731-740 (2016).

42. Monera, O. D., Sereda, T. J., Zhou, N. E., Kay, C. M. \& Hodges, R. S. Relationship of sidechain hydrophobicity and a-helical propensity on the stability of the single-stranded amphipathic a-helix. J. Pept. Sci. 1, 319-329 (1995).

43. Ling, S. et al. Combined approaches of EPR and NMR illustrate only one transmembrane helix in the human IFITM3. Sci. Rep. 6, 24029 (2016).

44. Berendsen, H. J. C., van der Spoel, D. \& van Drunen, R. GROMACS: a message-passing parallel molecular dynamics implementation. Comput. Phys. Commun. 91, 43-56 (1995).

45. de Jong, D. H. et al. Improved parameters for the Martini coarse-grained protein force field. J. Chem. Theory Comput. 9, 687-697 (2013).

46. Yen, H.-Y. et al. Ptdlns $(4,5) P_{2}$ stabilizes active states of GPCRs and enhances selectivity of G-protein coupling. Nature 559, 423-427 (2018).

47. Hsu, P.-C. et al. CHARMM-GUI Martini maker for modeling and simulation of complex bacterial membranes with lipopolysaccharides. J. Comput. Chem. 38, 2354-2363 (2017).

48. Qi, Y. et al. CHARMM-GUI Martini maker for coarse-grained simulations with the Martini force field. J. Chem. Theory Comput. 11, 4486-4494 (2015).

49. Berendsen, H. J. C., Postma, J. P. M., van Gunsteren, W. F., DiNola, A. \& Haak, J. R. Molecular dynamics with coupling to an external bath. J. Chem. Phys. 81, 3684-3690 (1984).

50. Tironi, I. G., Sperb, R., Smith, P. E. \& van Gunsteren, W. F. A generalized reaction field method for molecular dynamics simulations. J. Chem. Phys. 102, 5451-5459 (1995).

51. Wassenaar, T. A., Pluhackova, K., Böckmann, R. A., Marrink, S. J. \& Tieleman, D. P. Going backward: a flexible geometric approach to reverse transformation from coarse grained to atomistic models. J. Chem. Theory Comput. 10, 676-690 (2014).
52. Huang, J. \& MacKerell, A. D. Jr. CHARMM 66 all-atom additive protein force field: validation based on comparison to NMR data. J. Comput. Chem. 34, 2135-2145 (2013).

53. Martyna, G. J., Klein, M. L. \& Tuckerman, M. Nosé-Hoover chains: the canonical ensemble via continuous dynamics. J. Chem. Phys. 97, 2635-2643 (1992).

54. Buchner, M. et al. Identification of FOXM1 as a therapeutic target in B-cell lineage acute lymphoblastic leukaemia. Nat. Commun. 6, 6471 (2015).

55. Kang, J. H. et al. Noninvasive monitoring of single-cell mechanics by acoustic scattering Nat. Methods 16, 263-269 (2019).

56. Burg, T. P. et al. Weighing of biomolecules, single cells and single nanoparticles in fluid. Nature 446, 1066-1069 (2007).

57. Juric, D. et al. Differential gene expression patterns and interaction networks in $B C R-A B L$ positive and -negative adult acute lymphoblastic leukemias. J. Clin. Oncol. 25, 1341-1349 (2007).

58. Rosenwald, A. et al. The proliferation gene expression signature is a quantitative integrator of oncogenic events that predicts survival in mantle cell lymphoma. Cancer Cell 3, 185-197 (2003).

59. The Cancer Genome Atlas Research Network. Genomic and epigenomic landscapes of adult de novo acute myeloid leukemia. N. Engl. J. Med. 368, 2059-2074 (2013).

Acknowledgements We thank M. A. Lemmon for critical discussion and advice, and current and former members of the Müschen laboratory for their support and helpful discussions. Research in the Müschen laboratory is funded by the NIH/NCl through R35CA197628, R01CA157644, R01CA213138 and P01CA233412, the Dr. Ralph and Marian Falk Medical Research Trust Transformational Awards Program through MCG-18447-19, the Leukemia and Lymphoma Society through MCL-7000-18 and Blood Cancer Discoveries Grant BCDG-20327-20, as well the Howard Hughes Medical Institute HHMI-55108547 (to M.M.). This work was partly supported by NIH grants R35CA200422, R01CA251275, Al116585, Al116585S, Al140718, Al140705, DE023926 and DE028521 (to J.U.J.), R01 Al104002 and R01 Al127513 (to M.S.D.), R01 GM117923 and R01 GM097261 (to N.V.), NCI R35 CA231958 (to D.M.W.), Wellcome Trust 110275/Z/15/Z (to T.P.M.) and the Paul G. Allen Frontiers Group and the Cancer Systems Biology Consortium funding (U54-CA217377) from the National Cancer Institute (to S.R.M.). Relevant research in the A.T.-T. laboratory was supported by the Cancer Moonshot grant U01 CA232563. T.S. is a fellow of the Lymphoma Research Foundation.V.K. is supported by a Career Development Fellow grant (5491-20) from the Leukemia and Lymphoma Society, and by a Young Investigator Award from Alex's Lemonade Stand Foundation. Proteomics services were performed by the Northwestern Proteomics Core Facility, generously supported by NCI CCSG P30 CA060553 awarded to the Robert H Lurie Comprehensive Cancer Center, instrumentation award (S10OD025194) from NIH Office of Director, and the National Resource for Translational and Developmental Proteomics supported by P41 GM108569. M.M. is a Howard Hughes Medical Institute (HHMI) Faculty Scholar. J.C. is a Leukemia and Lymphoma Society (LLS) Scholar.

Author contributions J.L. performed experiments and contributed to all aspects of the study; in particular, western blotting, flow cytometry analysis, viable cell counts, growth competition assays, immunization assays, ELISA, colony-forming assays, CRISPR-mediated gene deletion, cell sorting, in vivo transplantation experiments, bio-imaging, bio-ID, immunoprecipitation assays, lipid strip assays, lipid raft analysis, calcium flux measurements and data analysis. M.E.R. and H.G. performed all bioinformatic and biostatistical analyses and power calculations for experimental design. N.M. and N.V. performed molecular dynamics simulations and structural analysis of IFITM3-PIP3 interactions. D.A. performed western blotting, flow cytometry analysis, viable cell counts, cell adhesion assays, CRISPR-mediated gene deletion and immunization assays. G.D., J.W., K.N.C., L.N.C., K.K. and V.K. performed flow cytometry analysis and viable cell counts. L.K. performed gene cloning, virus generation and flow cytometry analysis and cell sorting. M.A.A. performed RNA isolation for RNA sequencing, western blotting and CRISPR-mediated gene deletion. G.X. performed RNA-sequencing analysis with $P$ ten ${ }^{f / / f t} \mathrm{ER}^{\top 2}$ and Cre-ER ${ }^{T 2}$ mouse models and measured PIP3:PIP2 ratios. T.S. performed PLA. V.K. also performed immunofluorescence staining after immunization assays C.W.C. and J.C. provided expertise in gene-editing experiments. M.A.N. and A.P.W. provided expertise in cell-surface proteomics. A.T.-T. provided expertise in CD19 signalling, and M.F. M.S.D. and J.U.J. contributed expertise and reagents in antiviral effector functions of IFITM3 T.P.M., Y.Z., D.M.W. and S.R.M. performed SNACS cell-stiffness assays. M.M. secured funding, developed the amplification loop concept and wrote the manuscript. All co-authors reviewed and edited the manuscript.

Competing interests M.S.D. is a consultant for Inbios, Vir Biotechnology and NGM Biopharmaceuticals, and on the Scientific Advisory Board of Moderna and Immunome. The Diamond laboratory at Washington University School of Medicine has received unrelated sponsored research agreements from Moderna, Vir Biotechnology, and Emergent BioSolutions.

\section{Additional information}

Supplementary information is available for this paper at https://doi.org/10.1038/s41586-020 2884-6.

Correspondence and requests for materials should be addressed to M.M.

Peer review information Nature thanks Marcus Clark, Paul Kellam, Michael Reth and the other, anonymous, reviewer(s) for their contribution to the peer review of this work. Reprints and permissions information is available at http://www.nature.com/reprints. 


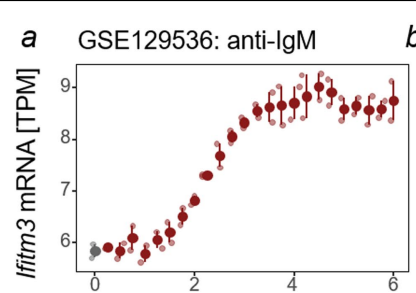

anti-IgM stimulation [hours]
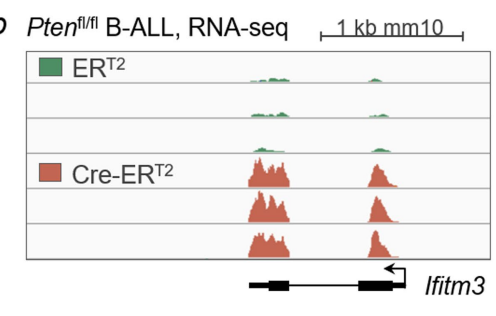

e

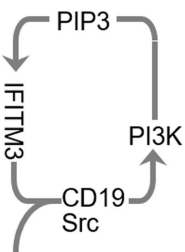

Antigen-encounter, oncogenic signaling

COG P9906 $\mathrm{n}=43 ; P=0.028$
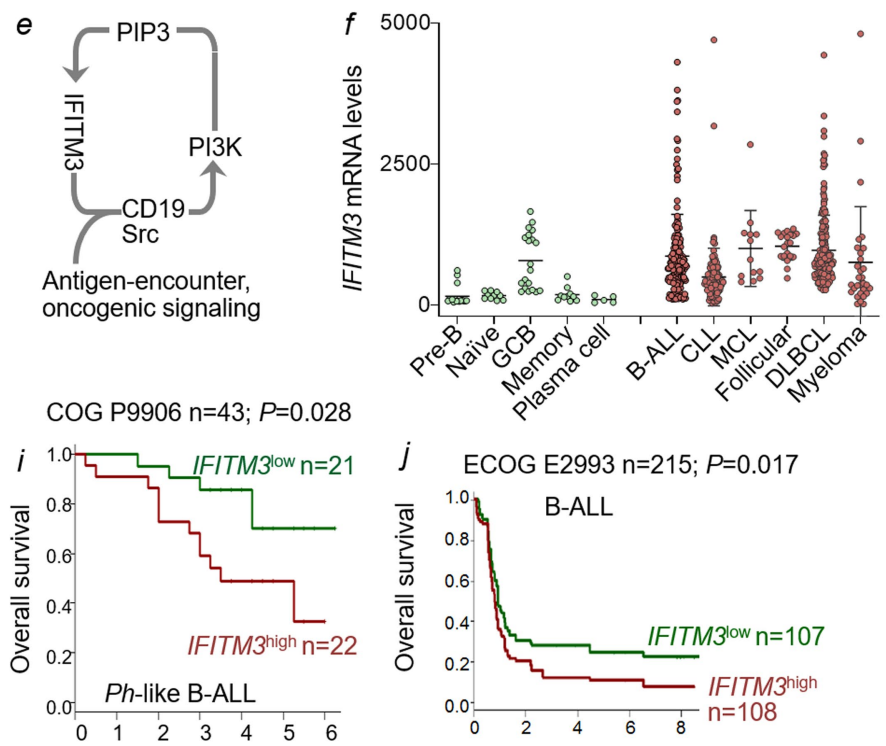

m Ikzf1exon5f//1 pre-B cells, ChIP-seq
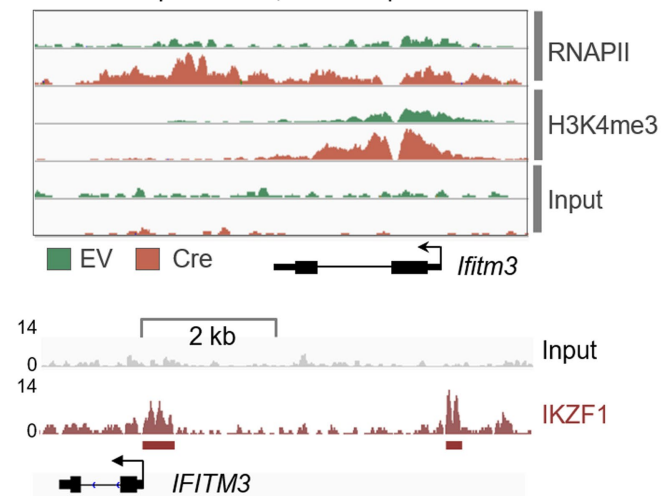

Extended Data Fig. 1 | Ifitm3 expression is induced by oncogenic PI3K signalling and repressed by IKZF1. a, Changes of Ifitm $3 \mathrm{mRNA}$ levels were monitored in mouse splenic $B$ cells upon BCR engagement (mean \pm s.e.; $n=2$ ). b, c, Pten $^{\text {flffl }}$ pre-B ALL cells carrying 4-OHT-inducible Cre-ER ${ }^{\mathrm{T} 2}$ or $\mathrm{ER}^{\mathrm{T} 2}$ were treated with 4-OHT and studied by RNA sequencing for Ifitm 3 transcript expression (b) and western blot for protein levels of PTEN, AKT-pS ${ }^{473}$, AKT and IFITM3 $(\mathbf{c} ; n=3)$. d, STAT5 phosphorylated at Y694 (STAT5-pY $\left.{ }^{694}\right)$, STAT5 and IFITM3 levels in patient-derived B-ALL cells (PDX2) measured by western blotting upon imatinib treatment $(n=3)$. e, Scenario of the PI3K pathway as a positive regulator of IFITM3, which in turn amplifies BCR and oncogenic signalling. f, IFITM3 mRNA levels across human normal and malignant haematopoietic and B-lymphoid samples (source data and statistics are given in Supplementary Table 2). Two-tailed $t$-test. $\mathbf{g}$, IFITM3 mRNA levels in pre-B cells from healthy donors and samples from patients with B-ALL were compared for two clinical cohorts (ECOG E2993 and St Jude). In ECOG E2993, bone marrow samples were obtained at diagnosis before treatment from 83 adults with B-ALL with a confirmed purity of $>90 \%$ leukaemic blasts. For the St Jude datasets, 15 samples from children with B-ALL before treatment were compared to flow-sorted pre-B cells from bone marrow aspirates of healthy donors. Two-tailed Wilcoxon. h, Minimal residual disease (MRD) was

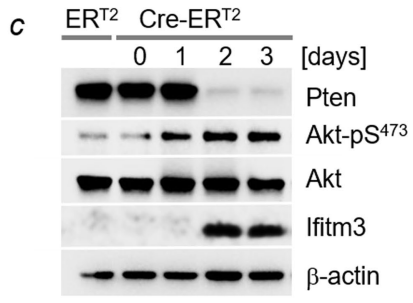

d Patient BCR-ABL1 B-ALL
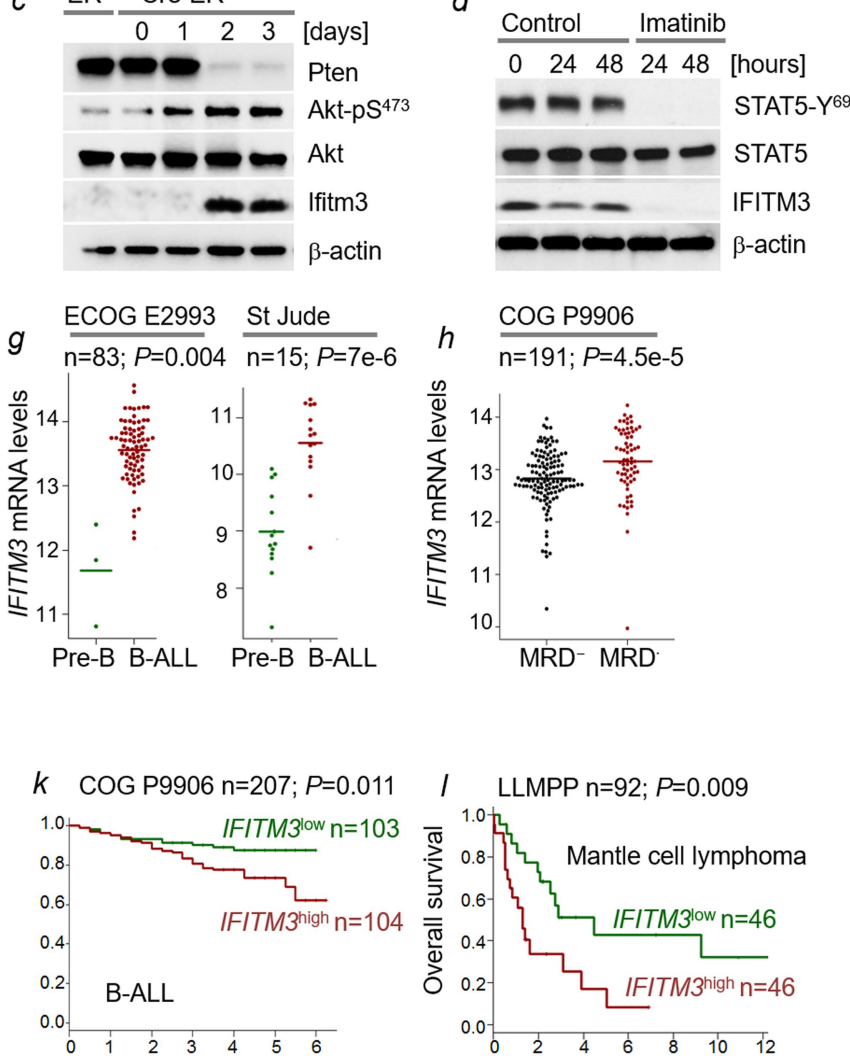

$n \quad P h^{+} \mathrm{ALL}+\mathrm{EV} \quad P h^{+} \mathrm{ALL}+\mid \mathrm{KZF} 1$

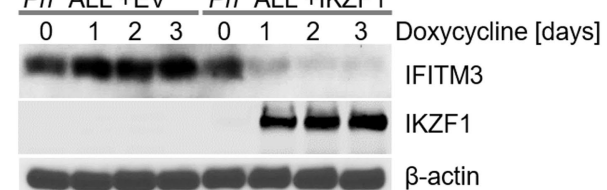

Multivariate analysis; COG P9906; $P=0.0045$

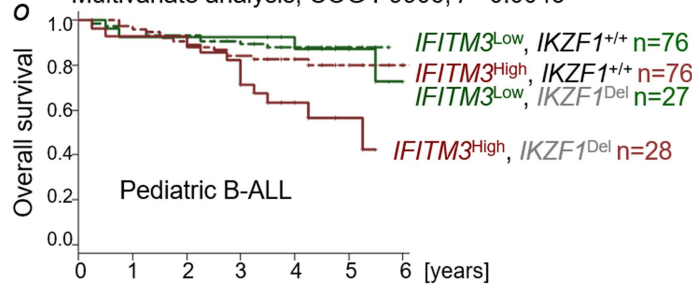

determined in the COG P9906 cohort, IFITM3 mRNA levels were compared in patients who were positive $(n=67)$ and negative $(n=124)$ for MRD. Two-tailed Wilcoxon. i-I, Patients with leukaemia and lymphoma from five clinical cohorts were segregated into two groups based on higher $\left(\right.$ IFITM ${ }^{\text {high }}$ ) or lower (IFITM3 ${ }^{\text {Iow }}$ ) than median IFITM3 mRNA levels. Overall survival was compared by two-tailed log-rank test. m, ChIP-seq enrichment of RNAPII and H3K4me3 at the Ifitm 3 locus in pre-B cells (top) from I $k z f 1^{\text {exonsflffl }}$ mice upon Cre-mediated deletion of $I k z f 1$ (GSE86897). Binding of IKZF1 to the promoter region of IFITM3 was also analysed in ChIP-seq data from patient-derived B-ALL cells (bottom, LAX2, GSE58825). n, Human B-ALL cells (BV173) carrying IKZF1 deletions were reconstituted with doxycycline-inducible IKZF1 or EV. Levels of IFITM3 were assessed by western blotting upon doxycycline-induction $(n=3)$. o, Multivariate analysis of established risk factors in children with B-ALL (COG P9906, $n=207)$ including mutation or deletion of $I K Z F 1$. Patients $(n=207)$ were separated into $I K Z F^{+++}$or $I K Z F$-deleted groups, then further segregated based on higher or lower than median expression levels of IFITM3. The comparison of these four groups established IFITM 3 mRNA levels as an independent risk factor regardless of $I K Z F 1$-deletion status (two-sided log-rank test; $P=0.0045$ ). c, d, n, For gel source data, see Supplementary Fig. 1 . 
a
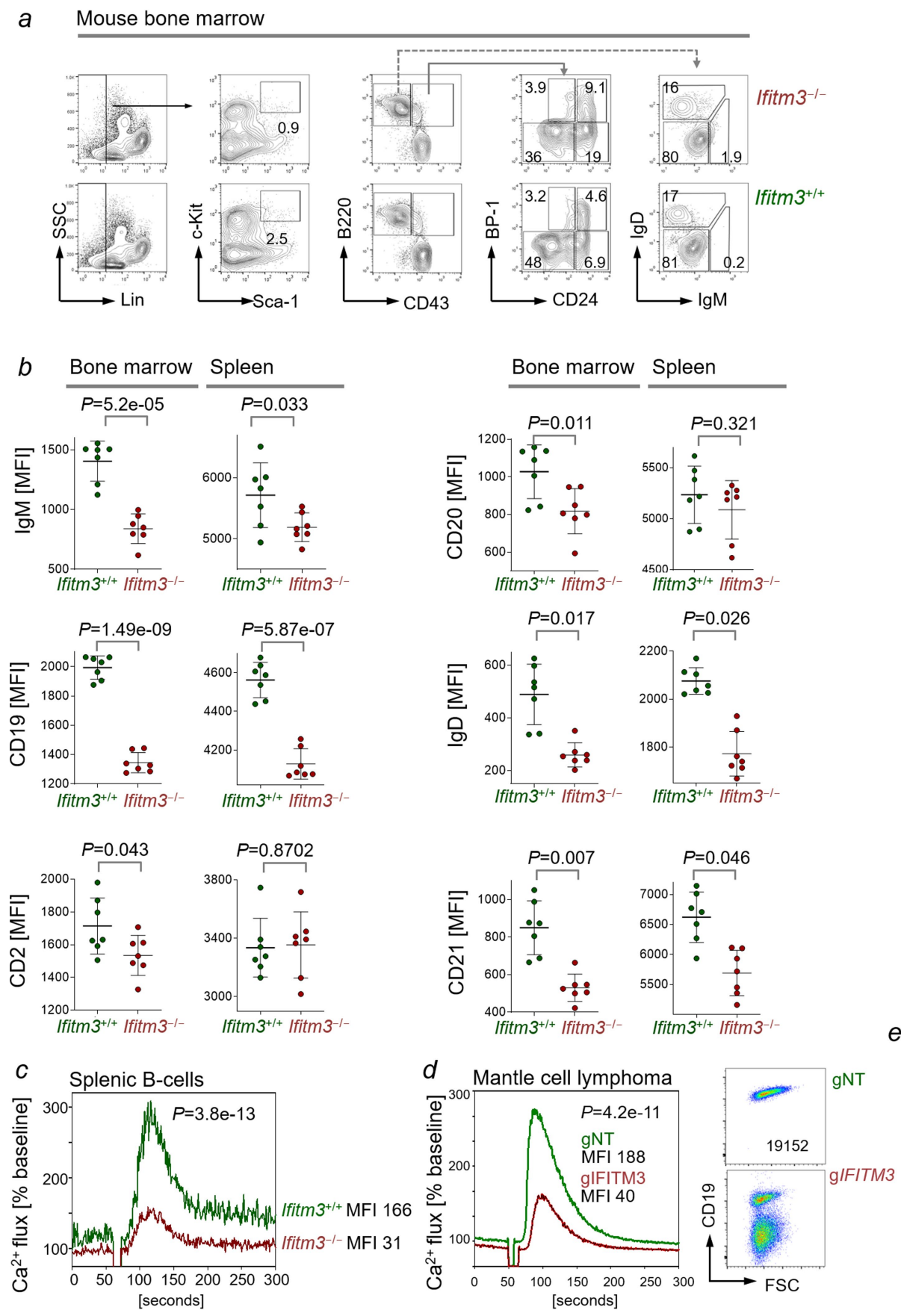
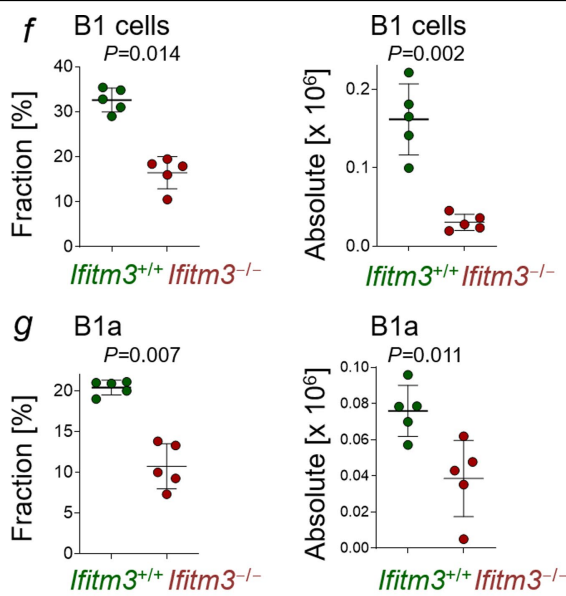

B1a

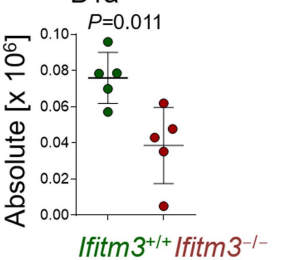

$h \quad \mathrm{MZB}$
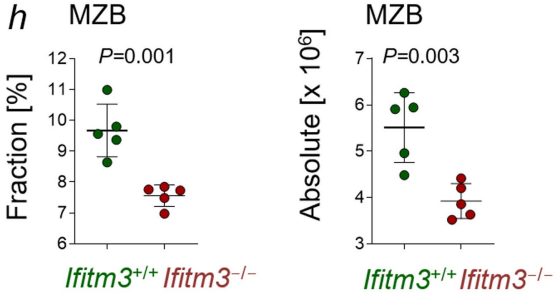

gNT gNT gIFITM3

e +Vehicle +Dasatinib +Vehicle

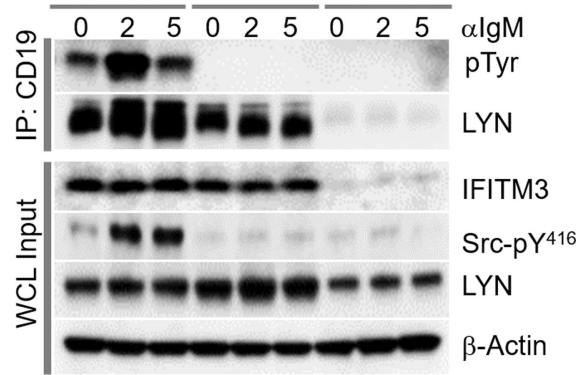

Extended Data Fig. 2 | Ifitm 3 is essential for the development of B1 and germinal centre $B$ cells. $a$, Hardy fractions of $B$ cell subsets isolated from bone marrow of $I$ fitm $^{+/+}$and Ifitm $^{-/-}$littermates analysed by flow cytometry $(n=3)$. b, Surface expression of IgM, CD20, CD19, IgD, CD2 and CD21 measured by flow cytometry in enriched bone marrow $\left(\mathrm{GR}^{-}, \mathrm{Nk}^{-1} 1^{-}\right.$and $\left.\mathrm{B} 22 \mathrm{O}^{+}\right)$and splenic $\mathrm{B}$ cells

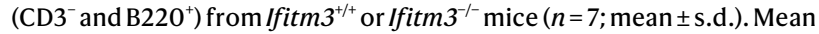
fluorescence intensities (MFI) values for individual measurement compared by two-tailed $t$-test. $c, \mathrm{Ca}^{2+}$ mobilization from cytoplasmic stores in response to $\mathrm{BCR}$ (IgM) engagement was measured in $\mathrm{Ifitm} 3^{+/+}$and $\mathrm{Ifitm} 3^{-/-}$splenic B cells. $\mathrm{Ca}^{2+}$ release was induced by addition of $10 \mu \mathrm{g} \mathrm{ml}^{-1}$ anti-mouse IgM $60 \mathrm{~s}$ after acquisition of background fluorescence. $\mathrm{Ca}^{2+}$ release was measured over $300 \mathrm{~s}$ with cell-permeant Rhod-2 dye; MFI compared between replicates $(n=3)$.d, $\mathrm{Ca}^{2+}$ mobilization in response to $\mathrm{BCR}$ engagement measured upon CRISPRCas9-mediated deletion of IFITM3 in Jeko1 MCL cells. $\mathrm{Ca}^{2+}$ release upon addition of $10 \mu \mathrm{g} \mathrm{ml}^{-1}$ of polyclonal $\mathrm{F}\left(\mathrm{ab}^{\prime}\right)_{2}$ anti-human IgM was measured for $300 \mathrm{~s}$ with cell-permeant Fluo-4 dye; MFI compared between replicates (left; $n=3$ ). Surface expression of CD19 following deletion of IFITM3 in Jeko1 MCL cells, MFIs for CD19 indicated (right; $n=3$ ). e, Jeko1 MCL cells were electroporated with non-targeting RNP (Cas9-gRNA ribonucleoproteins, gNT) or IFITM3-targeting RNP complex (gIFITM3). Following electroporation, MCL cells were treated with vehicle (DMSO) or $25 \mathrm{nmoll}^{-1}$ of dasatinib for $3 \mathrm{~h}$. Cells were stimulated with $10 \mu \mathrm{g} \mathrm{ml}^{-1}$ of anti-human $\operatorname{IgM~F}\left(\mathrm{ab}^{\prime}\right)_{2}$ for the indicated time points and subjected to co-immunoprecipitation with an anti-CD19 antibody. Immunoblots were performed to measure levels of CD19 tyrosine phosphorylation and binding of LYN to CD19. Levels of IFITM3, SRC-pY $\mathrm{Y}^{416}$ and LYN were assessed in whole-cell lysates (10\% input) with $\beta$-actin as loading control. ( $n=3$; gel source data in Supplementary Fig. 1$) . \mathbf{f}-\mathbf{h}$, Relative fractions (left) and absolute cell counts (right) of total B1 (f) and B1a (g) cells in the peritoneal cavity and marginal-zone B cells $(\mathbf{h})$ in spleen of $/$ fitm $^{3 / /+}$ and $I$ fitm $^{-/-}$ littermates $(n=5)$ are shown (means \pm s.d.; two-tailed $t$-test). 


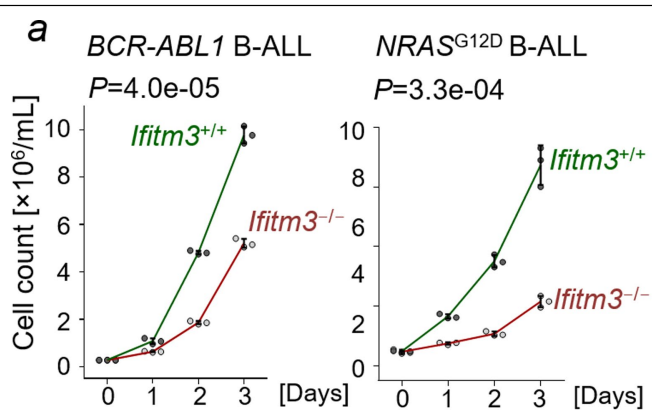

$b$
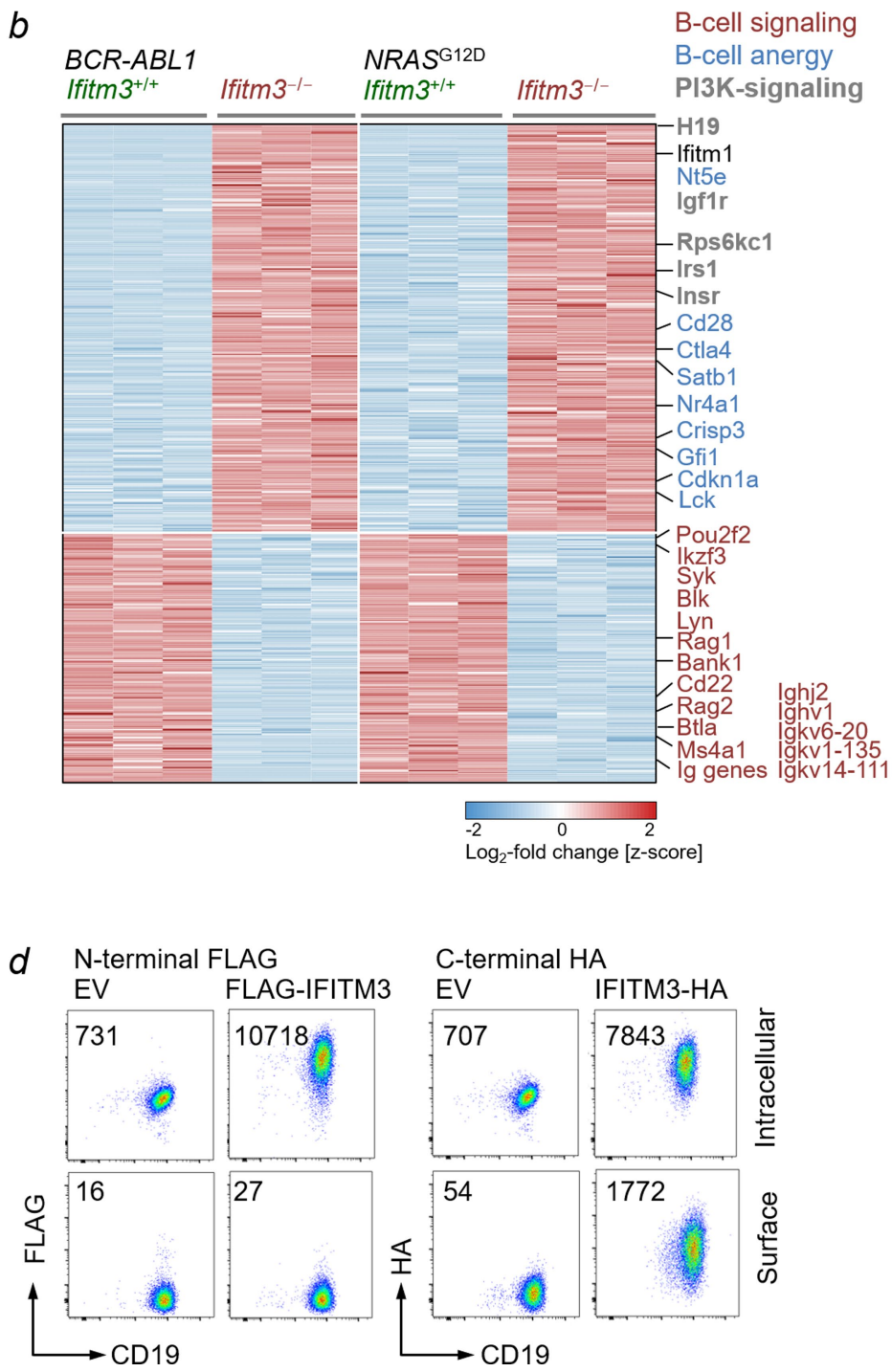

Extended Data Fig. 3 | Ifitm3-deficient B-ALL cells exhibit an anergic phenotype and compensatory upregulation of Ifitm1 and PI3K signalling molecules. a, Numbers of viable $I$ fitm $^{+/+}$and $I$ fitm $3^{-/-} B C R-A B L 1$ or $N R A S^{G 12 D}$ B-ALL cells were counted by Trypan blue dye exclusion $(n=3$; mean \pm s.d.; two-tailed $t$-test). b, RNA sequencing was performed for $I$ fitm $3^{+/+}$and $I$ fitm $3^{-/-}$ $B C R-A B L 1$ and $N R A S^{G 12 D} \mathrm{~B}-\mathrm{ALL}$ cells. Relative rlog-normalized gene expression values for all strongly differentially expressed genes $\left(P<1 \times 10^{-5}\right.$ and $\log _{2}$-transformed fold change $>1$; Wald test with Benjamini-Hochberg correction) in both $B C R-A B L 1$ and $N R A S^{G 12 D}$ conditions plotted as a heat map with row scaling. $B$-cell-signalling-related genes are labelled in red, anergy-related genes are labelled in blue and PI3K-signalling-related genes are labelled in grey.c, Gene set enrichment analysis for genes ranked by ratio of Ifitm $3^{-/-}$to $/$fitm $^{+/+}$as $\log _{2}$-transformed fold change; red lines indicate running enrichment score (right axis), grey bars indicate fold change (left axis).

\section{BCR-ABL1 B-ALL \\ Ifitm $^{+/+}$Ifitm $3^{-1-}$}

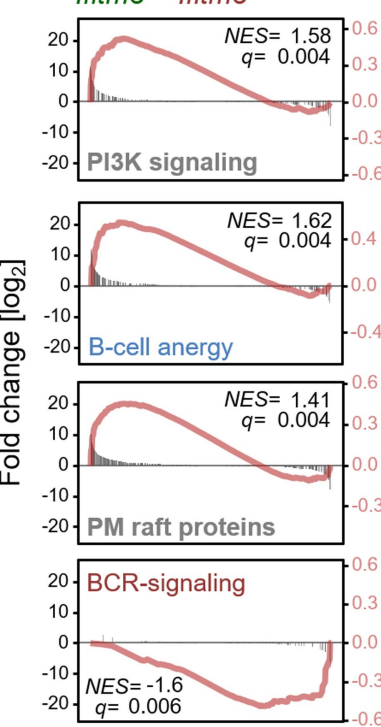

NRAS $S^{\mathrm{G} 12 \mathrm{D}} \mathrm{B}-\mathrm{ALL}$

Ifitm $3^{+/+}$Ifitm $^{-1}$
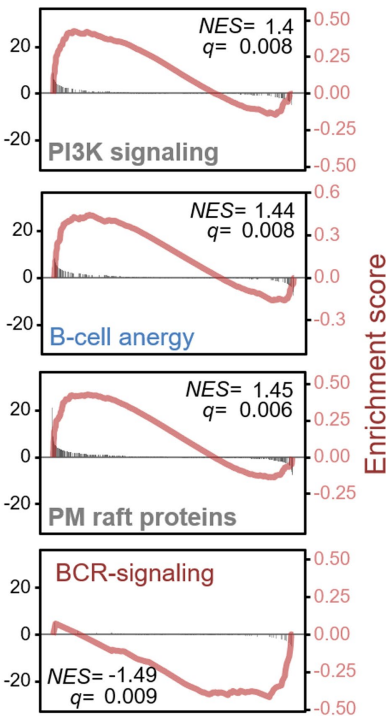

Src (LYN) Src-inhibitor Phospho-mimetic BCR-ABL1 Dasatinib Dasatinib HA

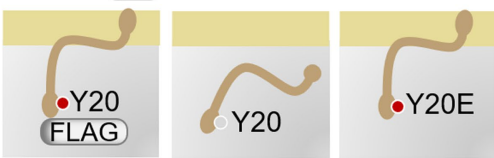

IFITM3-HA IFITM3-HA Y20E-HA
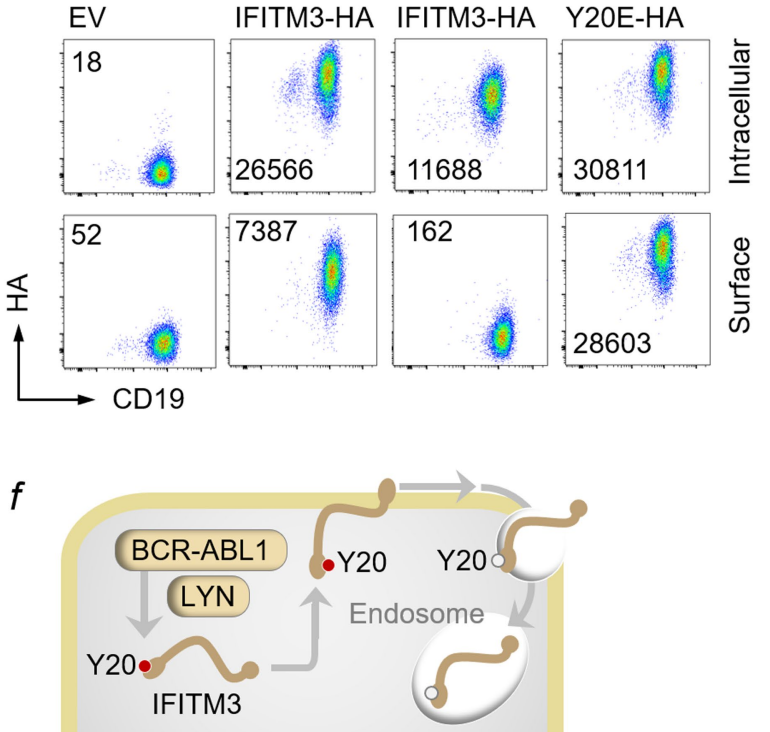

Statistical significance was determined by two-tailed Kolmogorov-Smirnov test. d, Patient-derived B-ALL cells (PDX2) were transduced with $\mathrm{N}$-terminally Flag-tagged or C-terminally HA-tagged IFITM 3 constructs. Combinations of intracellular and surface staining were performed to examine IFITM 3 topology at the cell membrane. e, Patient-derived B-ALL cells (PDX2) were transduced with C-terminal HA-tagged IFITM3 or the IFITM3(Y2OE) phosphomimetic. Combinations of intracellular and surface staining, with or without SRC-kinase inhibition by dasatinib, were performed to examine IFITM 3 topology at the cell membrane and its regulation by SRC kinases. $\mathbf{d}, \mathbf{e}$, Representative plots from three independent experiments. $f$, A scenario of the topology of IFITM3 regulated by SRC (LYN) or oncogenic tyrosine kinases (BCR-ABL1) at the plasma membrane is shown. Phosphorylation of $Y 20$ hinders the recognition of ${ }^{20} \mathrm{YEM}^{23}$ endocytosis motif by the AP-2 complex, thereby antagonizing endocytosis and endosomal trafficking of IFITM3. 


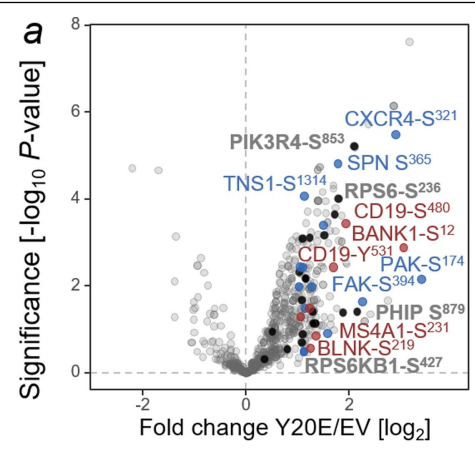

$C$ T-ALL anti-CD3E
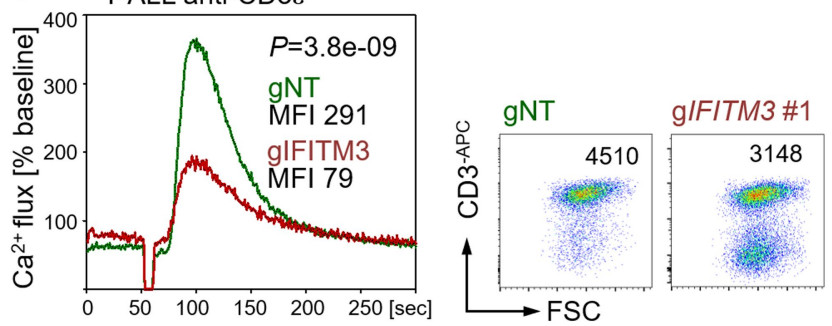

e

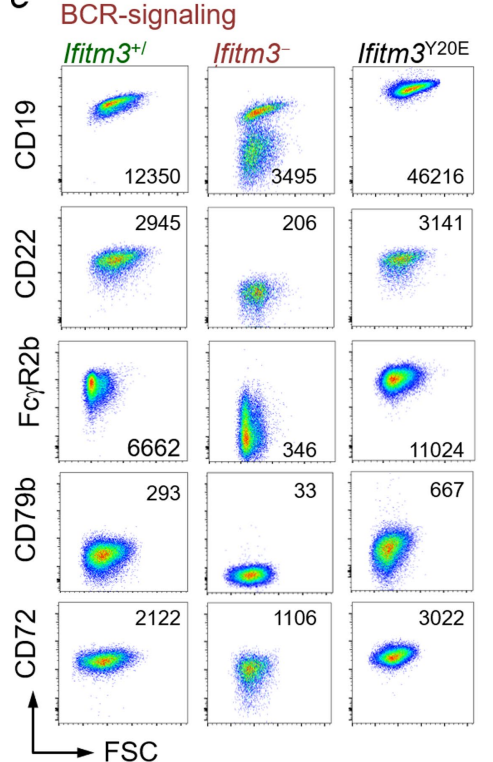

FC
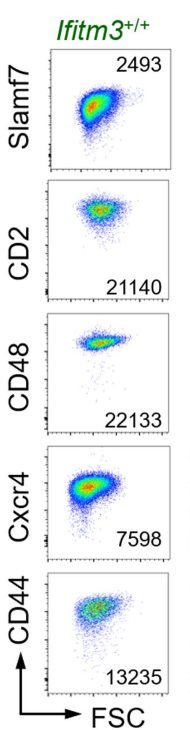

BCR-signaling
Integrin
PI3K-signaling

$b$

Whole aHA $F(a b) a H A$ Whole Ab $F(a b)$

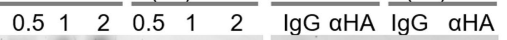

$=0$

$-m-m=$

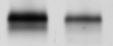

Whole aHA IgG: $1 \mu \mathrm{g} / \mu \mathrm{l}$ aHA $F(a b): 0.18 \mu \mathrm{g} / \mu \mathrm{l}$
EV IFITM3 Y20E EV IFITM3 Y20E

$\frac{-+-+-+\frac{-+1+++}{\text { Full Ab }}}{\text { F(ab) fragment }}$ anti-HA

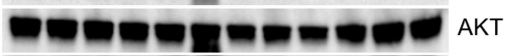
201. $-2=0$ IFITM3

అயா $\beta$-Actin d Ifitm $3^{+/+}$vs Ifitm $3^{-/-}$B-ALL cells

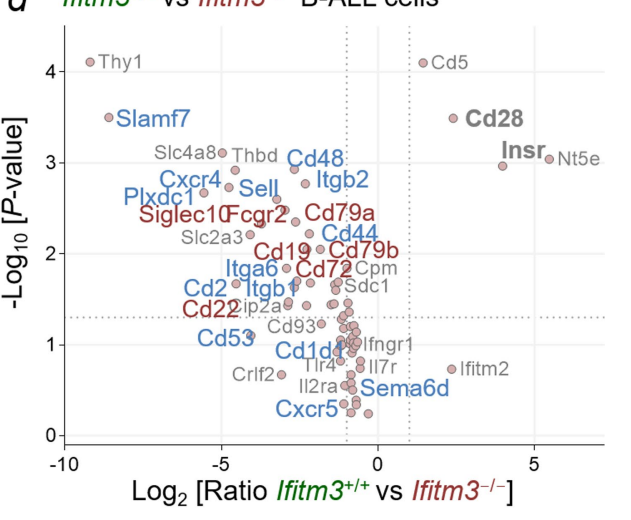

$f$ Adhesion and integrin receptors
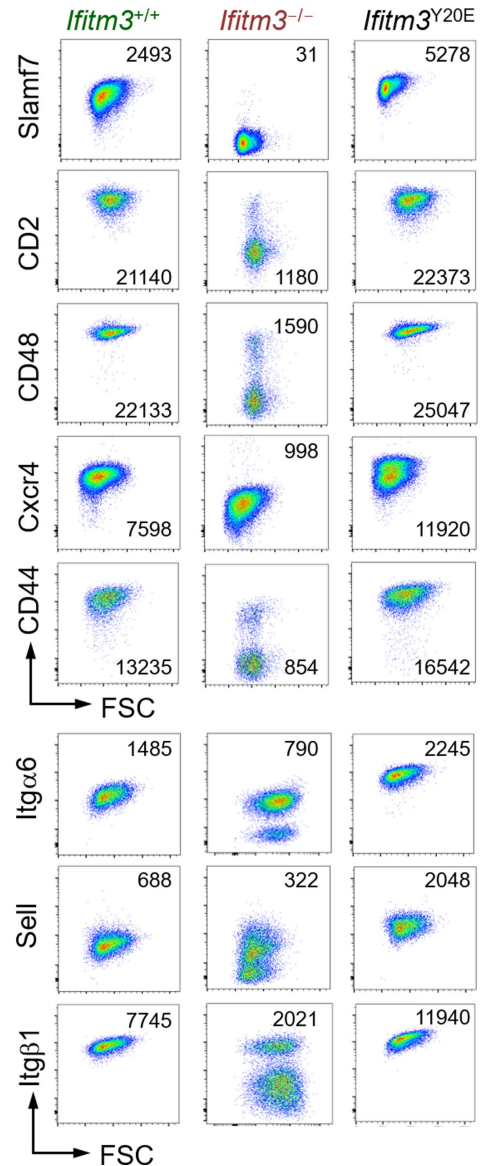

g
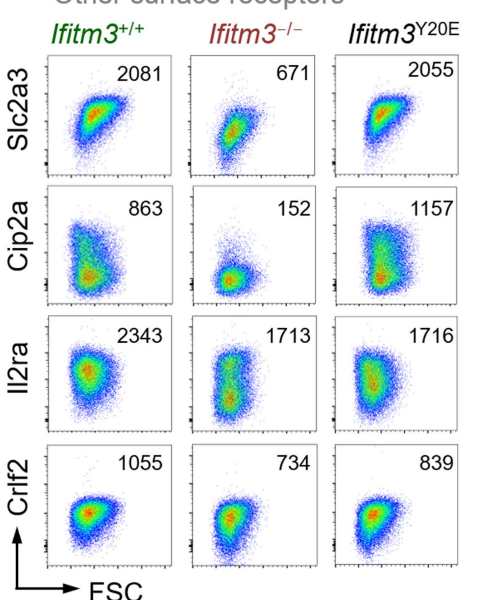

Extended Data Fig. 4 | See next page for caption. 


\section{Article}

Extended Data Fig. 4 | IFITM3 amplifies PI3K signalling downstream of BCR and integrin receptors. a, Volcano plot of differentially phosphorylated proteins in patient-derived B-ALL (PDX2) cells transduced with IFITM3(Y2OE) compared to empty vector (EV) control ( $n=3$; Wald test with BenjaminiHochberg correction). $\mathbf{b}, \mathrm{F}(\mathrm{ab})$ fragments of the anti-HA antibody or isotype control were purified and their identity confirmed by western blot (left). Eight million patient-derived B-ALL (PDX2) cells carrying IFITM3-HA, IFITM3(Y2OE)$\mathrm{HA}$ or EV control were resuspended in complete medium and treated with either $2.5 \mu \mathrm{g} \mathrm{ml}^{-1}$ of full antibodies, $\mathrm{F}(\mathrm{ab})$ fragments of anti-HA or isotype control for the indicated times. Levels of AKT- $\mathrm{pS}^{473}$, AKT and HA-tagged IFITM3 were assessed by western blots using $\beta$-actin as loading control (right). Data from three independent experiments. For gel source data, see Supplementary Fig. 1.c, $\mathrm{Ca}^{2+}$ mobilization in response to TCR engagement using $\mathrm{CD} 3 \varepsilon$-specific antibodies was measured upon CRISPR-Cas9-mediated deletion of IFITM3 in T-ALL cells (Jurkat) (left). $\mathrm{Ca}^{2+}$ release from cytoplasmic stores was induced by adding $10 \mu \mathrm{g} \mathrm{ml}^{-1}$ of monoclonal (OKT3) anti-human CD3 $\varepsilon$ at $50 \mathrm{~s}$ after acquisition of background fluorescence. Surface expression of CD3 was measured following deletion of IFITM3 in Jurkat cells (right). MFIs for CD3 are indicated. Representative plots from three independent biological experiments are shown. MFI values for individual measurement were compared by two-tailed $t$-test. d, Surface proteins on $/$ fitm $^{+/+}$and $I$ fitm $3^{-/-}$ B-ALL cells were labelled with biotin and enriched with streptavidin affinity pull-down followed by on-bead trypsin digestion, mass spectrometry and quantified with label-free quantification. Differentially expressed cell surface proteins on Ifitm $3^{+/+}$and $I$ fitm $3^{-/-}$B-ALL cells are shown with the mean difference of label-free quantification plotted against the $P$ value (Welch's $t$ test). All experiments were performed in biological triplicates. $\mathbf{e}-\mathbf{g}$, Validation of differential expression of surface receptors between f $_{\text {itm }} 3^{+/+}$, Ifitm $3^{-/-}$and Ifitm $3^{Y_{2 O E}}$-overexpressing B-ALL cells. Flow cytometry analyses show surface expression of BCR signalling components (e), integrins and adhesion receptors (f) and other surface receptors (g) in Ifitm $3^{+/+}$, Ifitm $3^{-/-}$and B-ALL cells expressing IFITM3(Y20E) $(n=3)$. 


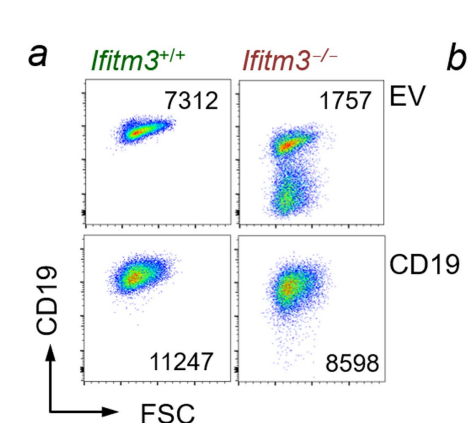

Ifitm $3^{+/+}$Ifitm $3^{-1-}$

EV CD19 EV CD19

$\rightarrow-\infty$ Akt-pS 473

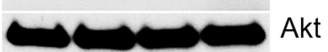

$=0$ Myc

$\longrightarrow \mathrm{Bcl} 2$

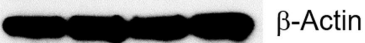

FSC

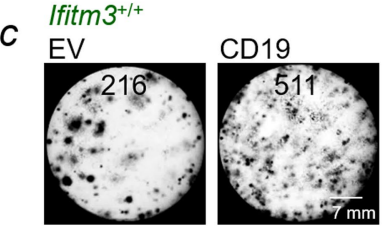

$P=0.004$
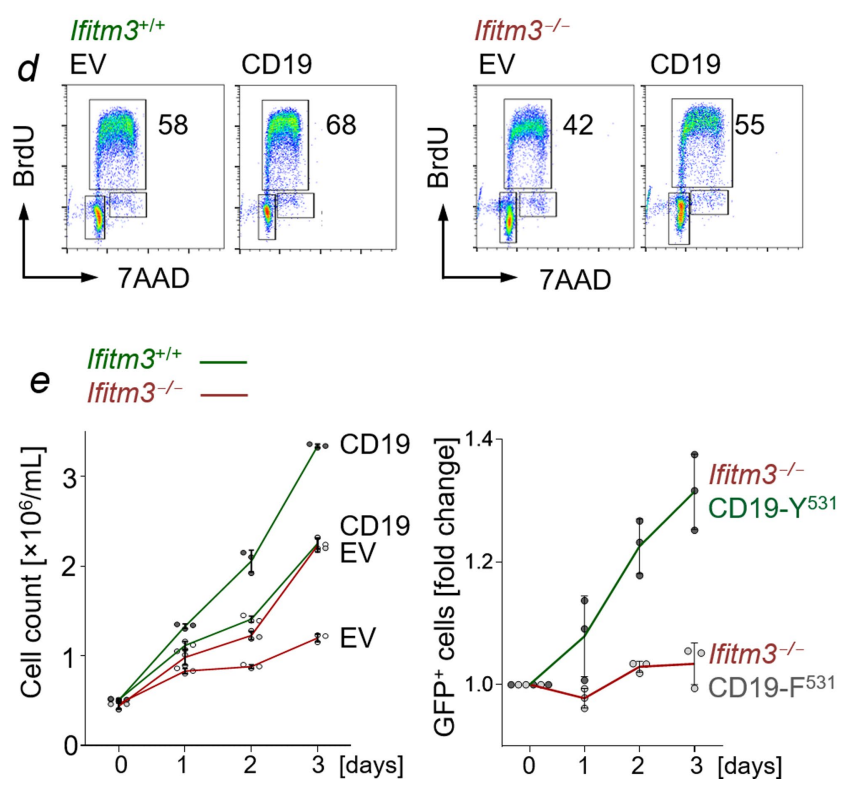

Extended Data Fig. 5 | Inducible membrane translocation of CD19 does not rescue defective SRC and PI3K signalling in Ifitm3-deficient B cells. a, Surface expression of $\mathrm{CD} 19$ was assessed by flow cytometry following forced expression of CD19 for $>1$ week in 1 fitm $^{\text {t/+ }}$ and fitm $^{-/-}$B-ALL cells. b. Western blot analyses of AKT-pS ${ }^{473}$, AKT, MYC and BCL2 upon forced expression of CD19 for $>1$ week in mouse fitm $3^{+/+}$and $/$fitm $^{-/-}$B-ALL cells. c, d, Colony-forming ability (c) and cell cycle progression (d) of Ifitm $3^{+/+}$and Ifitm $3^{-/-}$B-ALL cells upon forced expression of $\mathrm{CD} 19$ for $>1$ week was examined. c, Colony numbers for individual measurement were compared by two-tailed $t$-test.d, Numbers indicate percentage of cells in S phase. e, Numbers of viable $\mathrm{Ifitm}^{+/+}$and $\mathrm{Ifitm3^{-/ }}$ B-ALL cells following forced expression of CD19 were counted using the Trypan blue dye exclusion method (left). Ifitm $3^{-1-}$ B-ALL cells were transduced with GFP-tagged constructs for expression of CD19 with an intact (Y531) or mutant (Y531F) PI3K-activation motif in its cytoplasmic tail. Relative changes of $\mathrm{GFP}^{+}$cells (transduced with CD19 or CD19(Y531F)) were plotted over time (means \pm s.d.). a-e, Data from three independent experiments.b, For gel source data, see Supplementary Fig. 1.f, Mouse $C d 19^{-/-}$B-ALL clones were generated by electroporation of mouse B-ALL cells with CD19-targeting RNP (Cas9-gRNA ribonucleoproteins, gCd19 ALL) and single-cell clones with biallelic deletion
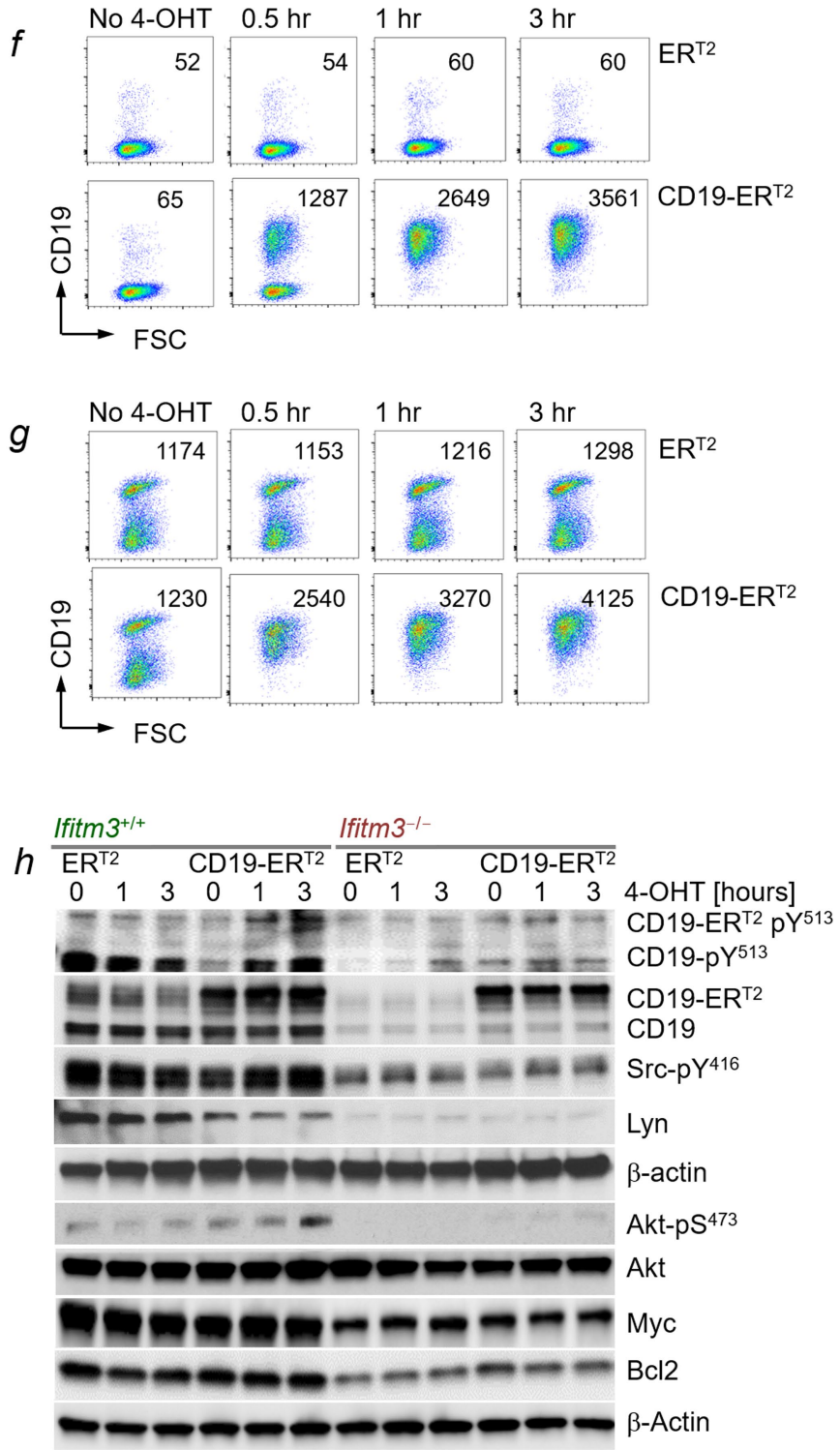

were selected. Cd19 ${ }^{-/-}$B-ALL cells were transduced with CD19-ER ${ }^{\mathrm{T} 2}$, a fusion of the ER-ligand binding domain to the $C$ terminus of $C D 19$, or $\mathrm{ER}^{\mathrm{T} 2}$ as empty vector control. Reconstitution of $C d 19^{-/-} \mathrm{B}$-ALL cells with CD19-ER ${ }^{\mathrm{T2}}$ resulted in stable expression of the fusion proteins that were retained in complex with cytoplasmic heatshock proteins. Addition of 4-OHT released CD19-ER ${ }^{\mathrm{T2}}$ from its cytoplasmic heatshock chaperone and enable cell surface expression within $30 \mathrm{~min}$ of $4-\mathrm{OHT}$ addition. g, To test the effect of inducible CD19 membrane translocation in

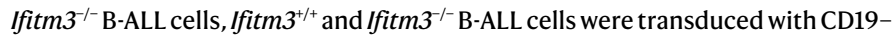
$\mathrm{ER}^{\mathrm{T} 2}$ or $\mathrm{ER}^{\mathrm{T} 2}$ empty vector control. 4-OHT-mediated translocation of $\mathrm{CD} 19$ to the cell surface was assessed by flow cytometry for the indicated times ( 0 to $3 \mathrm{~h}$ ). $\mathbf{h}$, Ifitm $^{+/+}$and fitm $^{-/-} \mathrm{B}$-ALL cells were transduced with CD19-ER ${ }^{\mathrm{T} 2}$ or $\mathrm{ER}^{\mathrm{T} 2}$ empty vector control. Cells were treated for 0,1 and $3 \mathrm{~h}$ with $4-\mathrm{OHT}$ for surface-translocation of CD19. Cell lysates from these populations were analysed by western blot for CD19-pY ${ }^{531}$, CD19, SRC-pY416, LYN, AKT-pS ${ }^{473}$, AKT, MYC and BCL2. While CD19-ER ${ }^{\mathrm{T} 2}$ reconstitutes CD19 protein levels in Ifitm ${ }^{-/-}$B-ALL cells and restores rapid translocation to the cell surface (g), this change alone was not sufficient to induce proper phosphorylation of CD19, SRC kinases and PI3K signalling via AKT. $\mathbf{f}-\mathbf{h}$, Data from three independent experiments. $\mathbf{h}$, For gel source data, see Supplementary Fig. 1. 


\section{Article}
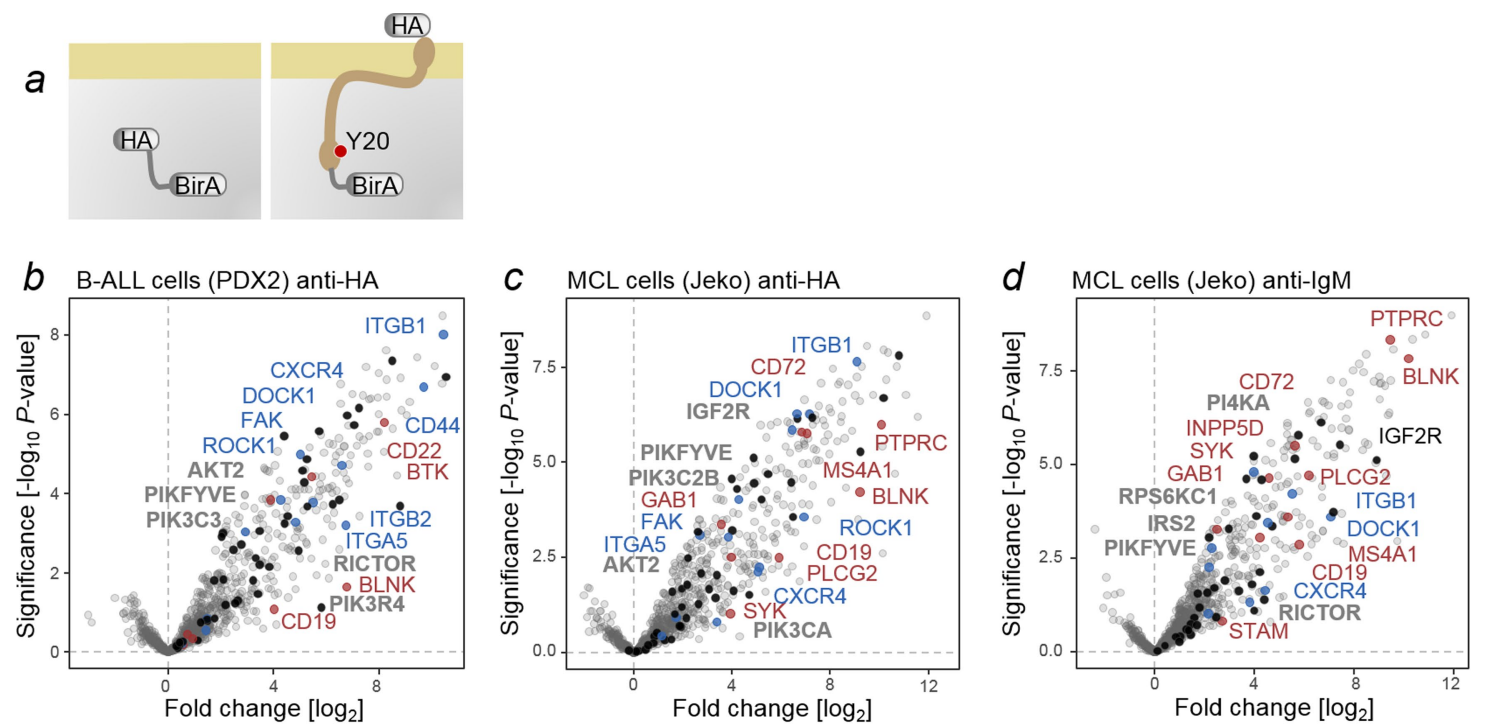

BCR-signaling Integrin $\bullet$ PI3K-signaling •

\section{e}

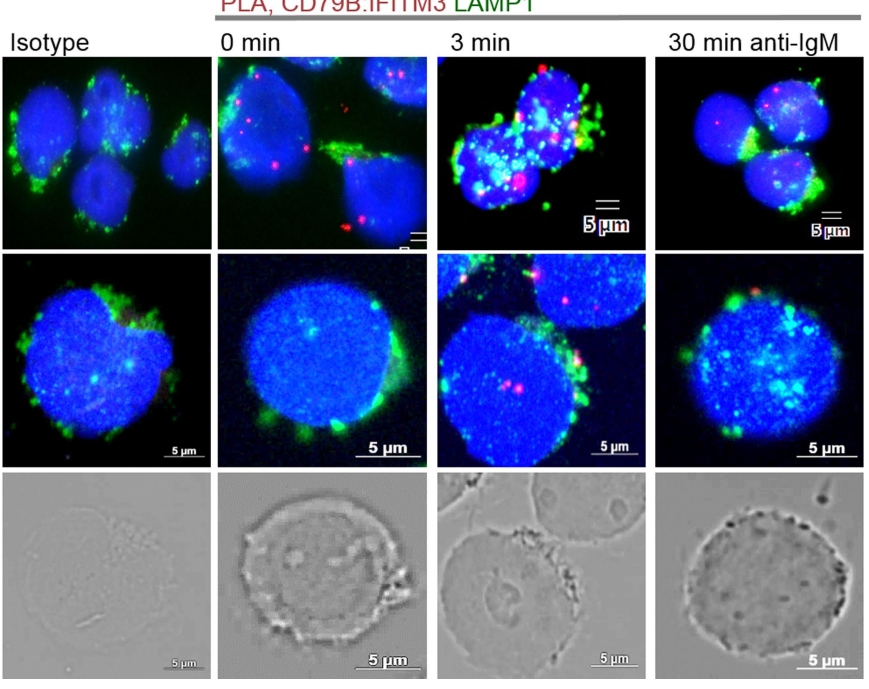

Extended Data Fig. 6 | IFITM3 links components of the BCR and integrin receptor pathways to PI3K signalling. a, Schematic of HA-tagged-IFITM3(Y2OE)BirA fusion proteins used for TurboID interactome analyses. BirA (biotin ligase) was fused to N-terminal IFITM3 carrying the phosphomimetic Y20E mutation for membrane localization. b-d, HA-IFITM3(Y2OE)-BirA or HA-BirA controls were expressed in PDX2 B-ALL (b) or Jeko1 MCL cells $(\mathbf{c}, \mathbf{d})$.c, d, Cells were incubated with exogenous biotin for 10 min upon IFITM3 (anti-HA) or BCR (anti-IgM) engagement. IFITM3(Y2OE) interactome analyses identified interacting proteins by mass spectrometry, plotted based on significance and $\log _{2}$-transformed fold enrichment over EV control. Essential interactors as BCR component (red), integrin (blue) and PI3K signalling (grey) are highlighted. Data from three independent biological replicates. e, PLAs were performed with Jeko1 MCL cells upon engagement of BCR. Jeko1 MCL cells were stimulated by BCR engagement for 0,3 and $30 \mathrm{~min}$, then fixed, permeabilized and assessed for the proximity of CD79B to IFITM3. Representative microscopic images with PLA signal (red dot) and nuclei stained with DAPI as blue are shown. LAMP1 was used as a marker for endosomes to distinguish plasma-membrane-bound from endosomal localization of CD79B:IFITM3 complexes. Scale bars, $5 \mathrm{~mm}$. Data from three independent replicates. 

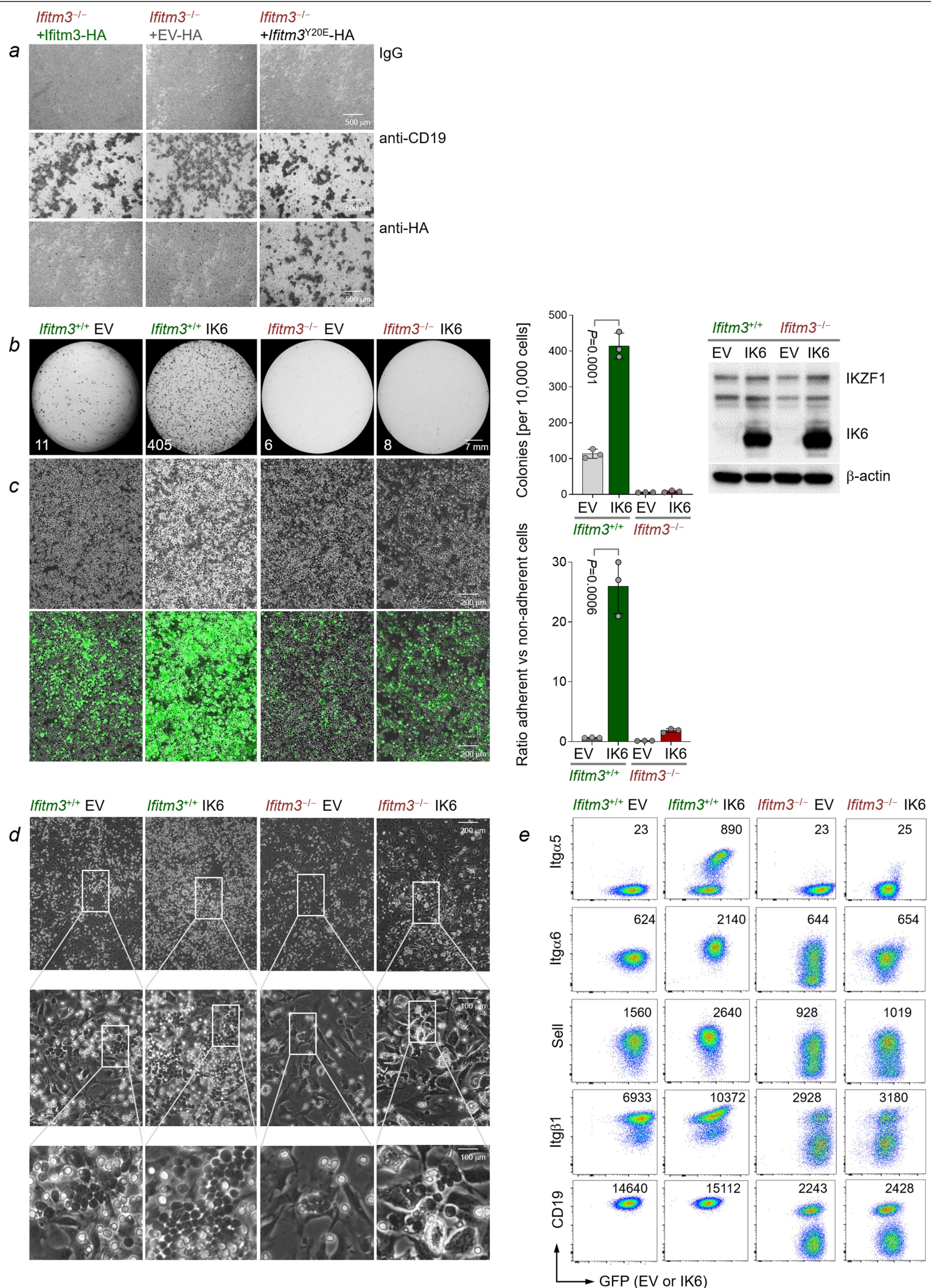

Extended Data Fig. 7 |See next page for caption. 


\section{Article}

Extended Data Fig. 7 | Ifitm3 functions as a central effector of B cell

adhesion. a, Homotypic aggregation was studied in 1 fitm $^{-1-}$ B-ALL cells that were reconstituted with C-terminal HA-tagged IFITM3, IFITM3(Y20E) or empty vector $(\mathrm{EV})$ and incubated with anti-CD19, anti-HA antibodies or isotype control for $24 \mathrm{~h}$. Data from three independent experiments. b, If $\mathrm{ftm}^{+/+}$or Ifitm $3^{-/-}$B-ALL cells were transduced with GFP-tagged IK6 or GFP alone. IK6 levels in flow-sorted $\mathrm{GFP}^{+}$cells assessed by western blot analysis using $\beta$-actin as loading control (right). Ten thousand $/$ fitm $^{+/+}{ }^{\text {or }}$ Ifitm $3^{-/-} \mathrm{B}$-ALL cells carrying IK6 or EV were plated for colony forming assays. Colonies were imaged and counted after 7 days. Representative images are shown with colony numbers. Data from three independent experiments and assessed by two-tailed $t$-test (means \pm s.d.). For gel source data, see Supplementary Fig.1.c, One hundred thousand Ifitm $3^{+/+}$or Ifitm $3^{-/-}$B-ALL cells carrying IK6 or EV were cultured on OP9 stroma cells. Ratios of adherent cells to nonadherent cells were calculated. Data from three independent biological experiments (right) were analysed and assessed by two-tailed $t$-test (means \pm s.d.).d, Representative images of adherent B-ALL cells on OP9 stroma are shown. Round and light-refracting cells are adherent B-ALL cells attached to stroma cells. Dark and round cells are adherent B-ALL cells incorporated into stromal layer. Data from three independent biological replicates. e, Surface expression levels of integrins on adherent B-ALL cells were measured by flow cytometry. MFI values are indicated for individual measurements. Data from three independent biological replicates. 

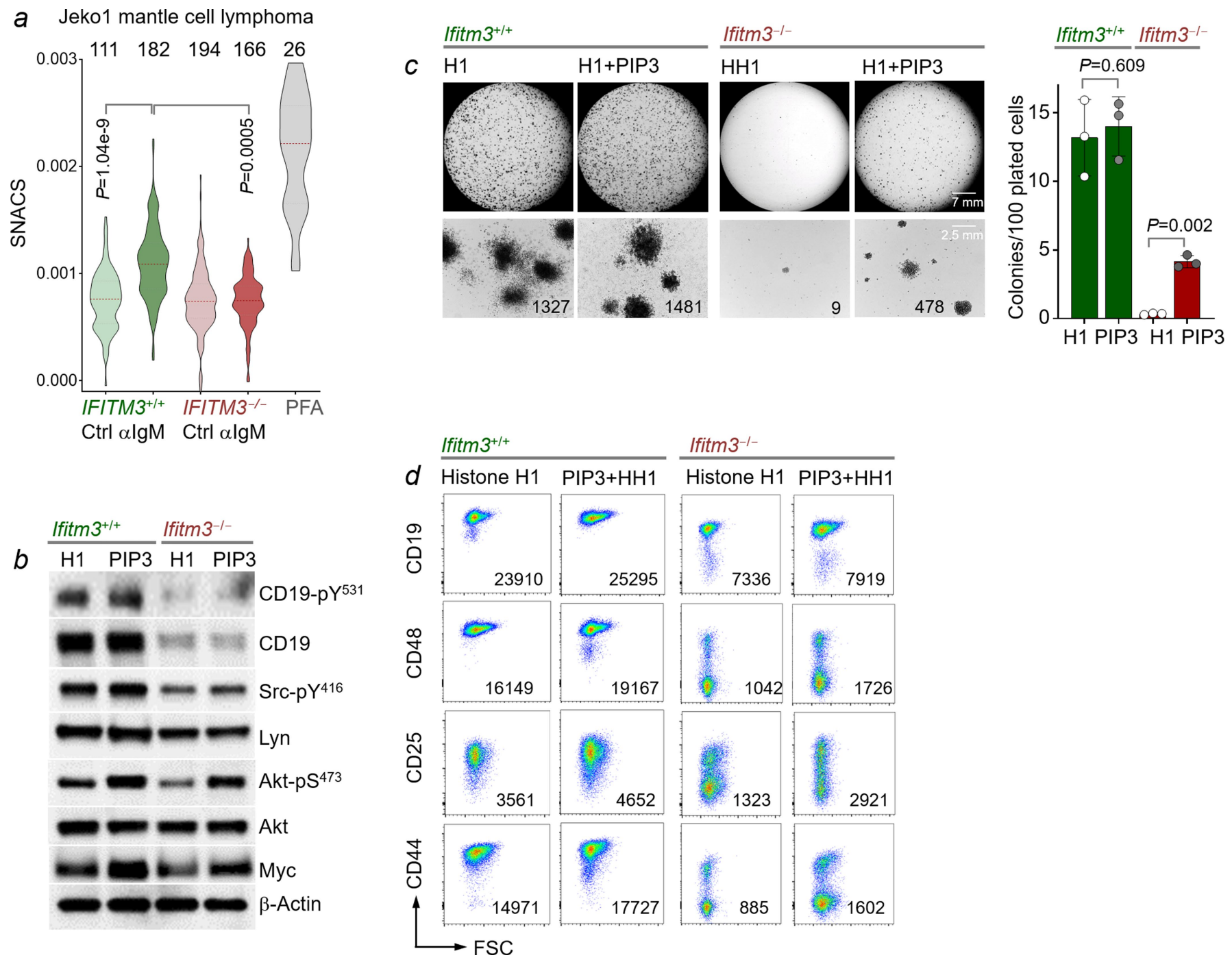

Extended Data Fig. 8 | IFITM3 functions as a PIP3 scaffold and mediated cell-membrane stiffening upon BCR engagement. a, SNACS was measured using a previously established microfluidic method as a metric for cell surface stiffness. Jeko1 mantle cell lymphoma cells were flown through a standing acoustic wave generated inside a vibrating suspended microchannel resonator. The cantilever vibration frequency was monitored, and its shift was used to quantify the acoustic scattering from the cells as well as the buoyant mass of the cells. The data displayed was obtained using a $350-\mu \mathrm{m}$-long cantilever with $15 \times 20-\mu \mathrm{m}$-sized channel inside of the cantilever and an approximately $200-\mathrm{ms}$ transit time through the cantilever. All the regulators, valves and data acquisition were controlled by custom software coded in LabVIEW 2017 (National Instruments). A parallel volume measurement using Coulter Counter was carried out to quantify average cell volume, which was used together with the single-cell buoyant mass measurements to calculate SNACS for each cell. Jeko1 mantle cell lymphoma cells without $\left(\right.$ IFITM $\left.^{+/+}\right)$and with $\left(\right.$IFITM3 $\left.^{-/}\right)$ CRISPR-Cas9-mediated deletion of IFITM3 were kept under cell culture conditions and treated with an anti-IgM antibody. Fixation with paraformaldehyde (PFA) was used as a positive control. Representative plots from three independent experiments are shown (median levels in red dotted line). Statistical significance was determined by two-tailed $t$-test. Numbers indicated cells studied for SNACS measurement. b, f $_{\text {itm }} 3^{+/+}$and $/$fitm $^{-/-} \mathrm{B}-\mathrm{ALL}$ cells were incubated with $30 \mu \mathrm{M}$ of the PIP3 carrier histone H1 or PIP3-histone H1 complex for 30 min. Levels of CD19-pY ${ }^{531}, \mathrm{CD} 19$, SRC-pY $Y^{416}$, LYN, AKT-pS ${ }^{473}$, AKT and MYC were measured by western blot using $\beta$-actin as loading control. Data from three independent replicates. For gel source data, see

Supplementary Fig. 1.c. Colony-formation assays were performed for $/$ fitm $^{+/ /+}$ and Ifitm $3^{-/-}$B-ALL cells that were treated with $30 \mu \mathrm{M}$ of PIP3-histone $\mathrm{H} 1$ or the shuttle protein histone $\mathrm{H} 1$ (H1) alone. Photomicrographs and colony numbers per 10,000 plated cells are shown. Data are presented as means \pm standard derivation (s.d.) from three independent experiments. Statistical significance was determined by two-tailed $t$-test. d, Flow cytometry analyses of surface expression of CD19, CD44, CD25 and CD44 on f $_{\text {itm }} 3^{+/+}$and fitm $^{-/-} \mathrm{B}$-ALL cells treated with $30 \mu \mathrm{M}$ of PIP3-histone $\mathrm{H} 1$ or the shuttle protein histone $\mathrm{H} 1(\mathrm{H} 1)$ alone for $72 \mathrm{~h}$. Data from three independent replicates. 


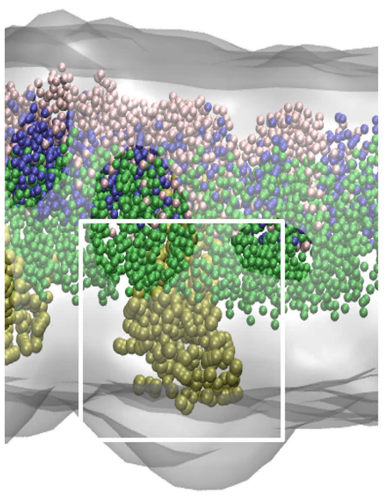

$b$

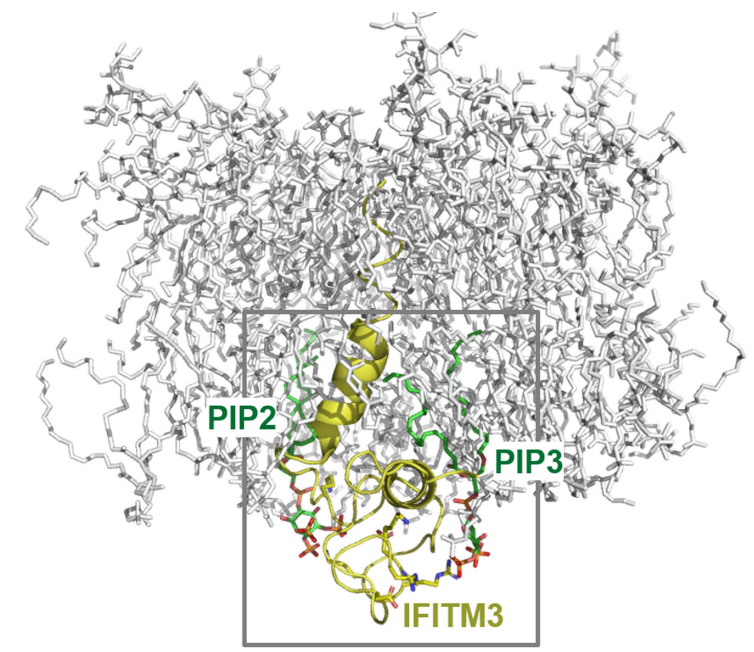

C

\section{PIP2}

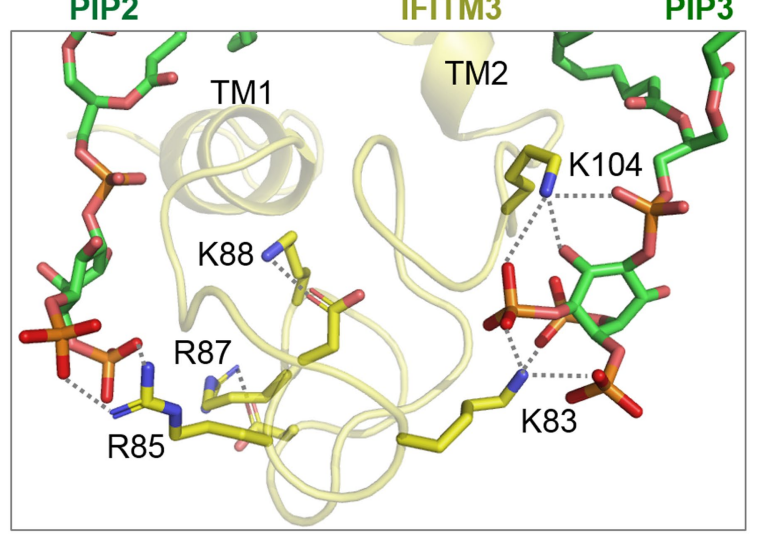

Conserved intracellular loop (CIL)

$\underline{\text { IM } \alpha \text {-helix }}$ LGFIAFAY
Extended Data Fig. 9 |Modelling of binding of IFITM3 to PIP2 and PIP3.

a-c, The hierarchical scheme of molecular dynamics simulations to delineate the structural and dynamical basis of PIP2 and PIP3 binding to IFITM 3 (yellow). a, Coarse grained simulations of IFITM 3 in composite cell membrane. The side views of the lipid bilayer are shown. The lipid raft markers are shown in colour with GM1 (pink), cholesterol (green) and sphingomyelin (blue). The grey surface represents all the other lipids. b. The simulation cell extracted from the coarse grained simulation as the starting structure for all-atom molecular dynamics simulations. The IFITM 3 protein is shown in yellow and the PIP2 and PIP3 are shown in green and red stick representations. c, Close up view of one of the predicted binding poses of PIP2 and PIP 3 in the most populated conformation of IFITM3. The dashed lines shown are the PIP2 or PIP3 contacts with the basic residues in IFITM3 (top). The amino acid sequence of IFITM3 in the stretch of residues between TM1 and TM 2 from 57 to 128 modelled in this work is shown. The CIL region is boxed and basic amino acids are highlighted with blue. The average interaction energy of PIP2 or PIP3 with the two basic batches measured in all-atom molecular dynamics simulations is indicated (Supplementary Table 6).d, e, The average interaction energy of PIP2 (green) or PIP3 (red) with IFITM3 (residues 57-128) was measured in all-atom molecular dynamics simulations. The one-residue contact indicates the conformation of PIP2 or PIP3 binding with only one basic residue in the basic amino patch KSRDRK of IFITM3. The two-residue contact refers to the binding conformation of PIP2 or PIP3 that show contacts with two basic residues. Representative plots from at least four independent experiments are shown (mean \pm s.d.). In d, $P$ values were determined by two-tailed $t$-test. Populations showing one- or two-residue contacts of PIP2 (green) or PIP3 (red) to IFITM3 were quantitated from all-atom molecular dynamics simulations. The population density was assessed by the normalization of number of events with the total number of frames. Representative plots from three independent experiments are shown (mean \pm s.d.). In e, statistical significance was determined by two-tailed $t$-test. 
a

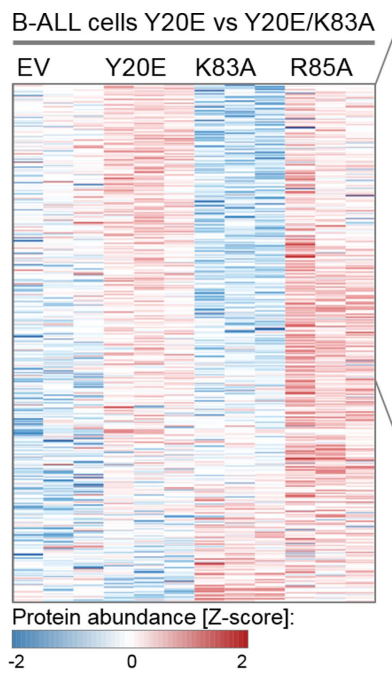

d

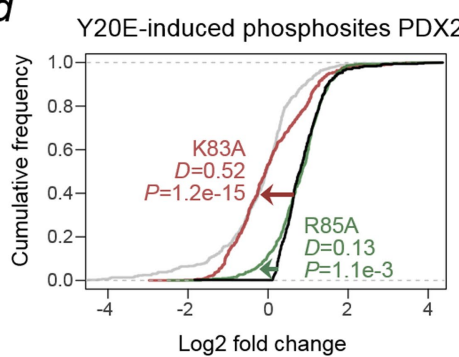

$b$

PHIP-S879
CD19-S $480 / S^{353}$

SPN-S363

PAK1-S174
BANK1-S12

CD19-Y531

PAG1-S150

PIIK3R4-S 853

RPSGKB1-S427

CD19-Y500

TNS1-S1314

MSA4A1-S231

PAG1-S50/S61

$\mathrm{CD} 19-\mathrm{S} 494 / \mathrm{S}^{497}$

CXCR4-S321

RICTOR-S1385

CD19-T538

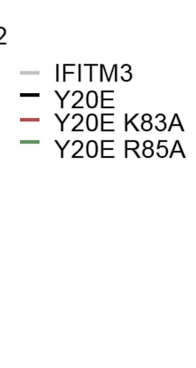

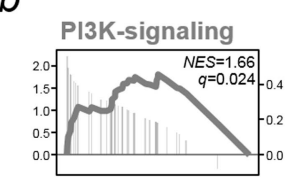
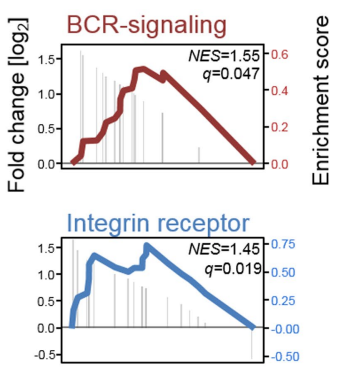

$\begin{array}{lll}\text { EV } & \text { Y20E } & \text { Y20E }\end{array}$

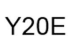

C B-ALL cells Y20E vs Y20E/R85A SPN-S365

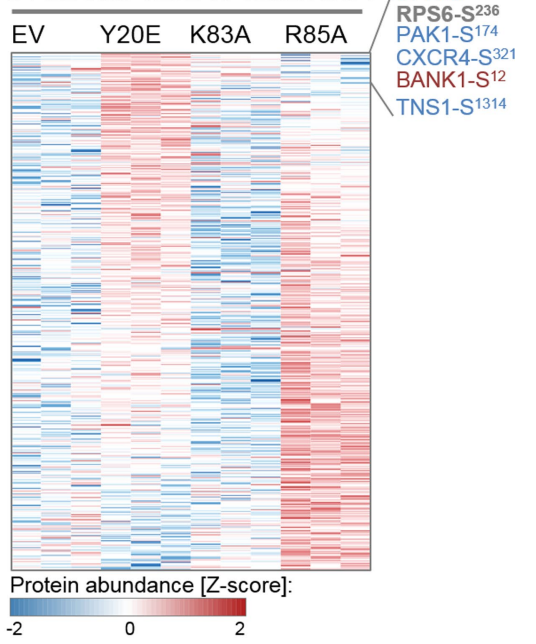

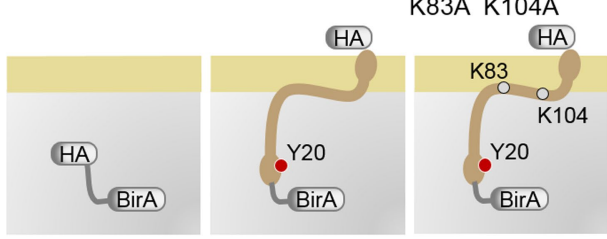

e

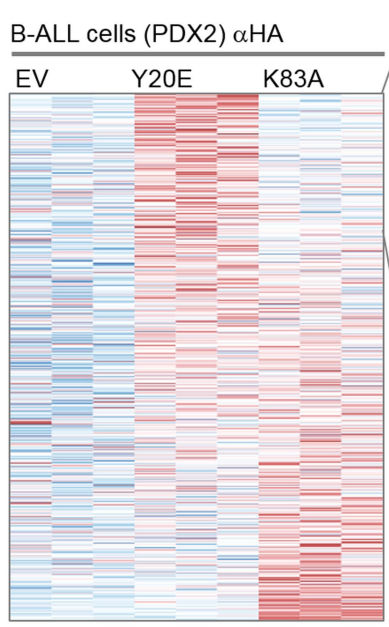

BCR-signaling •

Integrin

PI3K-signaling •

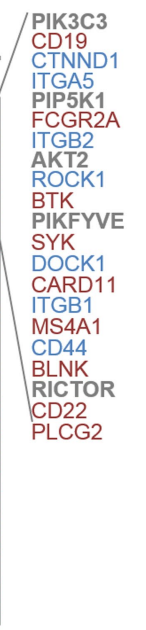

$f$
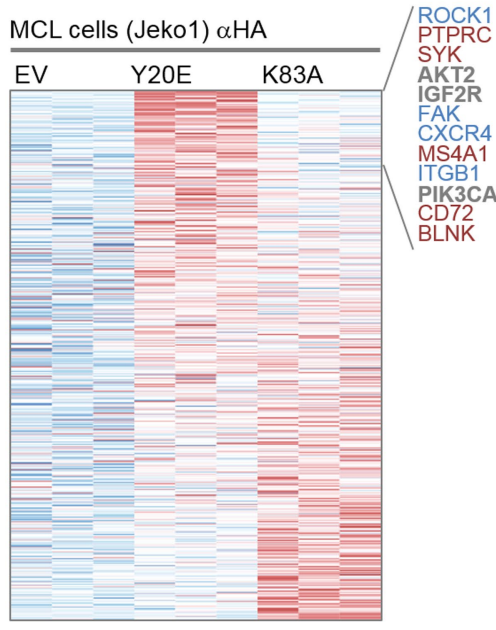

$g$

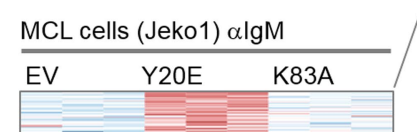
IGF2R
MS4A1 RPS $6 K C 1$ RICTOR RICTOR
CXCR4 CXCR4 STIM1 DOCK1 CD72 ROCK2
Extended Data Fig. 10 | IFITM3-mediated PI3K signalling downstream of $B C R$ and integrin receptors depends on K83-K104 but not on R85, R87 and $\mathrm{K88}$ residues. $\mathbf{a}-\mathbf{c}$, Levels of differentially phosphorylated proteins in patient-derived B-ALL (PDX2) cells transduced with IFITM3(Y2OE), IFITM3(Y2OE/K83A), IFITM3(Y2OE/R85A) or empty vector (EV) control were identified by mass spectrometry $(n=3)$. Relative abundance values are plotted for all sites ranked by fold change as indicated; phosphosites of interest are highlighted.b, FSEA ranked by $\log _{2}$-transformed fold change are shown for phosphosites in PI3K signalling (grey), BCR signalling (red) and integrin and adhesion receptor elements (blue). Statistical significance was determined by two-tailed Kolmogorov-Smirnov test.d, Cumulative distribution frequencies for $\log _{2}$-transformed fold changes in phosphosite abundance between IFITM3-transduced and EV conditions were calculated for all sites and globally increased in IFITM3(Y2OE) over EV. The analysis was repeated for the K83A (red) and R85A (green) mutants of IFITM3. The light grey line indicates background variance observed between EV replicates. Shifts that were caused by the $\mathrm{K} 83 \mathrm{~A}$ and $\mathrm{R} 85 \mathrm{~A}$ mutants are indicated by arrows. Overall changes in distribution (shifts) between IFITM3(Y2OE) and IFITM3(Y20E/K83A) and IFITM3(Y20E/R85A) mutants were measured by two-tailed KolmogorovSmirnov test. e, Schematic of BirA (engineered biotin ligase) was fused at $\mathrm{N}$-terminal ends of HA-tagged IFITM3(Y2OE) or its K83A mutant and expressed in PDX2 B-ALL or Jeko1 MCL cells. e-g, Interactomes of IFITM3(Y2OE) and its K83A mutant were compared in PDX2 B-ALL cells upon IFITM3 engagement (anti-HA) (e) or in Jeko1 MCL cells upon IFITM3 (anti-HA) (f) or BCR (anti-IgM) (g) engagement. Phosphosites of interest, including BCR signalling, PI3K signalling and integrins and adhesion receptor elements are highlighted. 


\section{natureresearch}

Corresponding author(s): Markus Müschen

Last updated by author(s): Aug 3, 2020

\section{Reporting Summary}

Nature Research wishes to improve the reproducibility of the work that we publish. This form provides structure for consistency and transparency in reporting. For further information on Nature Research policies, seeAuthors \& Referees and theEditorial Policy Checklist.

\section{Statistics}

For all statistical analyses, confirm that the following items are present in the figure legend, table legend, main text, or Methods section.

n/a Confirmed

$\boldsymbol{x}$ The exact sample size $(n)$ for each experimental group/condition, given as a discrete number and unit of measurement

$\boldsymbol{x}$ A statement on whether measurements were taken from distinct samples or whether the same sample was measured repeatedly

$\mathbf{x}$ The statistical test(s) used AND whether they are one- or two-sided

Only common tests should be described solely by name; describe more complex techniques in the Methods section.

$\mathbf{x} \square$ A description of all covariates tested

$\mathbf{x}$ A description of any assumptions or corrections, such as tests of normality and adjustment for multiple comparisons

A full description of the statistical parameters including central tendency (e.g. means) or other basic estimates (e.g. regression coefficient)

x AND variation (e.g. standard deviation) or associated estimates of uncertainty (e.g. confidence intervals)

For null hypothesis testing, the test statistic (e.g. $F, t, r$ ) with confidence intervals, effect sizes, degrees of freedom and $P$ value noted

$x$ Give $P$ values as exact values whenever suitable.

$\mathbf{x} \square$ For Bayesian analysis, information on the choice of priors and Markov chain Monte Carlo settings

$\boldsymbol{x} \square$ For hierarchical and complex designs, identification of the appropriate level for tests and full reporting of outcomes

$\boldsymbol{x} \square$ Estimates of effect sizes (e.g. Cohen's $d$, Pearson's $r$ ), indicating how they were calculated

Our web collection on statistics for biologists contains articles on many of the points above.

\section{Software and code}

Policy information about availability of computer code

Data collection

For Proximity ligation assay, images were acquired by CellSens imaging software (Olympus) with Zeiss Axio Observer 7 microscope. For FACS, BD FACSDIVA ${ }^{\text {TM }}$ SOFTWARE were used. For Western blot, images were collected by film exposure, UVP BioSpectrum 810 Imaging System or ChemiDoc MP Imaging System (BioRad). For colony forming assay, images were acquired GelCount analyzer and packages QCapture pro 7 connected with Olympus IX71 microscope. To determine the number of viable cells, the trypan blue exclusion method was applied, using the Countess II FL Automated Cell Counter. For in vivo bioimaging, IVIS 100 bioluminescence/optical imaging system was used. The IFITM3 protein structure was modeled by coarse-grained (CG) molecular dynamics simulation method in GROMACS (http:// www.gromacs.org/) with Martini force field. The three snapshots extracted from the CG simulations were converted to an all-atom system using Martini tools. The all-atom simulations were performed using GROMACS packageand CHARMM force field, with TIP3 water model. To set up the mixed lipid bilayer with water in the upper and inner regions, CHARMM-GUI was used. For immunohistology of immunized or non-immunized spleen sections, images were acquired on a ZEISS LSL 880 confocal microscope and analyzed on ZEN 2.3 (Zeiss) software.

For cell membrane stiffness, the Single-cell size-normalized acoustic scattering (SNACS) was measured by custom software coded in LabVIEW 2017 (National Instruments). A parallel volume was measured with Coulter Counter (Beckman Coulter) to quantify average cell volume.

Data analysis

For analysis of Proximity ligation assay, ImageJ, BlobFinder, Excel and GraphPad Prism 7 were used. For FACS analysis, FlowJo, SigmaPlot and GraphPad Prism 7 was used. For colony forming assay, number of colonies were counted by GelCount analyzer and analyzed by SigmaPlot. For in vivo leukemia burden analysis, GraphPad Prism 7 was used. For gene expression analysis, Excel, SigmaPlot and GraphPad Prism 7 was used. For log-rank test to compare survival differences between patient groups, R package 'survival' Version 2.35-8 and Cox proportional hazards regression model in R package for the multivariate analysis (https://www.r-project.org/) were used. Integrative Genomics Viewer (IGV) was used to visualize ChIP-seq tracks.

For RNA-seq analysis raw sequence reads were mapped to the mouse genome $(\mathrm{mm} 10)$ using STAR V2.5.39, and the frequency of genes was counted using featureCounts v1.5.110. The raw counts were then normalized using the trimmed mean of M values (TMM) method and compared using Bioconductor package "edgeR". Reads per kilo base per million (RPKM) mapped reads were also calculated from the 
raw counts. For differential expression analysis, transcripts were quantified using Salmon v1.1.0 against gencode GRCm38 vM24 transcript annotations; normalisation and statistical analysis was done in R using DESeq2 v1.28.1. Differentially expressed genes were identified if RPKM $\geq 1$ in at least one sample, fold change $\geq 2$, and $P \leq 0.05$. RPKM data were later used in the Gene set enrichment analysis. GSEA analysis was performed using the DOSE package in R12, genes were ranked by log2 fold-change, gene sets were obtained from MSigDB or from internal data as indicated.

For Bio-ID data analysis, proteins were identified from the MS raw files using Mascot search engine (Matrix science). MS/MS spectra were searched against the SwissProt human database. All searches included carbamidomethyl cysteine as a fixed modification and oxidized Met, deamidated Asn and GIn, acetylated N-term as variable modifications. Three missed tryptic cleavages were allowed. The MS1 precursor mass tolerance was set to $10 \mathrm{ppm}$ and the MS2 tolerance was set to 0.6 Da. A 1\% false discovery rate cutoff was applied at the peptide level. Only proteins with a minimum of two peptides above the cutoff were considered for further study. For comparison to empty vector control, background peptide abundances for missing values were imputed from a Gaussian distribution centered around the minimum observed abundance using the MinProb method from MSnbase package in $\mathrm{R}$.

For proteomics, raw spectral data was analyzed using MaxQuant v1.5.1.2 to identify and quantify peptide abundance and searched against the human Swiss-Prot annotated human proteome from Uniprot (downloaded with 20,303 entries). The "match-between-runs" option was selected to increase peptide identifications while the "fast LFQ" option was selected to calculate label-free quantification values (LFQ) of identified proteins. All other settings were left to the default MaxQuant values. The MaxQuant output data was analyzed using Perseus and the R program (version 3.4.0). Proteins annotated as "reverse", "only identified by site", and "potential contaminant" were filtered out as well as proteins that were quantified in less than 2 out of 3 biological replicates in at least one experimental group. Missing values were imputed based on the normal distribution of the dataset as implemented by Perseus. Volcano plots were generated using output from a two-sample t-test comparing the log2 transformed LFQ protein abundance values from different cell lines with a false discovery rate (FDR) set to 0.01 .

For phospho-proteomic analysis, $15 \mathrm{mg}$ total protein for each sample was digested with trypsin, and 500 ug total protein for each sample was digested with LysC/trypsin for IMAC analysis. Samples were purified over C18 columns and dried in a lyophilizer. Dried samples were resuspended and enriched with Fe-IMAC beads, purified over C18 STAGE tips (Rappsilber). Replicate injections of each sample were run non-sequentially on the instrument. Peptides were eluted using 150-minute (IMAC) linear gradient of acetonitrile in $0.125 \%$ formic acid delivered at $280 \mathrm{~nL} / \mathrm{min}$. Tandem mass spectra were collected in a data-dependent manner with a Thermo Orbitrap Fusion ${ }^{\mathrm{TM}}$ Lumos $^{\mathrm{TM}}$ Tribrid $^{\mathrm{TM}}$ mass spectrometer using a top-twenty MS/MS method, a dynamic repeat count of one, and a repeat duration of 30 sec. Real time recalibration of mass error was performed using lock mass (Olsen) with a singly charged polysiloxane ion $\mathrm{m} / \mathrm{z}=371.101237$. MS/MS spectra were evaluated using SEQUEST and the Core platform from Harvard University (Eng, Huttlin, Villen). Files were searched against the Swiss Prot Homo sapiens FASTA database. A mass accuracy of $+/-5$ ppm was used for precursor ions and 0.02 Da for product ions. Enzyme specificity was limited to trypsin, with at least one tryptic (K- or R-containing) terminus required per peptide and up to four miscleavages allowed. Cysteine carboxamidomethylation was specified as a static modification, oxidation of methionine and phosphorylation on serine, threonine, and tyrosine residues were allowed as variable modifications. Reverse decoy databases were included for all searches to estimate false discovery rates, and filtered using a 1\% FDR in the Linear Discriminant module of Core. Peptides were also manually filtered using a -/+5ppm mass error range and presence of a phosphorylated residue. All quantitative results were generated using Skyline (MacLean) to extract the integrated peak area of the corresponding peptide assignments. Accuracy of quantitative data was ensured by manual review in Skyline or in the ion chromatogram files.

For manuscripts utilizing custom algorithms or software that are central to the research but not yet described in published literature, software must be made available to editors/reviewers. We strongly encourage code deposition in a community repository (e.g. GitHub). See the Nature Research guidelines for submitting code \& software for further information.

\section{Data}

Policy information about availability of data

All manuscripts must include a data availability statement. This statement should provide the following information, where applicable:

- Accession codes, unique identifiers, or web links for publicly available datasets

- A list of figures that have associated raw data

- A description of any restrictions on data availability

Patient-outcome data for B-ALL were obtained from the National Cancer Institute TARGET DATA Matrix of the Children's Oncology Group (COG) Clinical Trial P9906 (GSE11877; Harvey et al., 2010; Kang et al., 2010), Eastern Cooperative Oncology Group (ECOG) Clinical Trial E2993 (GSE5314; Juric et al., 2007) and St. Jude Children's Research Hospital (https://www.stjuderesearch.org/site/data/ALL3/; Ross et al., 2003). Patient-outcome data for mantle cell lymphoma were obtained from https://Impp.nih.gov/MCL/ 32. Patient-outcome data for AML were obtained from TCGA Acute Myeloid Leukemia Project (http://www.cbioportal.org/study/ summary?id=laml_tcga_pub\#clinical; Cancer Genome Atlas Research Network, 2013). Proteomics data was deposited to the ProteomeXchange Consortium via the PRIDE partner repository with following accession numbers: cell surface proteome PXD014691, phosphoproteome PXD020696 and IFITM3 interactomes PXD020697. IFITM3 mRNA levels across human normal and malignant B-lymphoid samples were obtained from http://Amazonia.transcriptome.eu/. All other data are available from the corresponding author upon reasonable request. Genome binding/occupancy profiling from WT and IKDN stromal adherent pre-B cells were obtained from GSE86897. Immunohistochemistry images for IFITM3 levels in normal or malignant B cells were obtained from The Human Protein Atlas https:// www.proteinatlas.org/. ChIP-seq data of the genome wide mapping of IKZF1 binding (ChIP-Seq) in human patient-derived B-ALL xenograft cells were obtained from GSE58825. ChIP-seq data of the genetic analysis of IKZF1 target genes (ChIP-Seq) and tumor suppressor function in BCR-ABL1+ pre-B ALL were obtained from GSE90656. RNA sequencing (RNA-Seq) data with Ifitm3+/+ and Ifitm3-/- BCR-ABL1 or NRASG12D B-ALL cells are available at GSE155305. RNA sequencing (RNA-Seq) data with Ptenfl/fl pre-B cells carrying 4-OHT-inducible Cre-ERT2 or ERT2 are available at GSE155618. All other data needed to evaluate the conclusions in the paper are available within the main text or supplementary materials. 


\title{
Field-specific reporting
}

Please select the one below that is the best fit for your research. If you are not sure, read the appropriate sections before making your selection.
$\mathbf{x}$ Life sciences
Behavioural \& social sciences
Ecological, evolutionary \& environmental sciences

For a reference copy of the document with all sections, see nature.com/documents/nr-reporting-summary-flat.pdf

\section{Life sciences study design}

All studies must disclose on these points even when the disclosure is negative.

Sample size For in vitro experiments, phenotypic analyses including Western blot and FACS were performed in at least three independent experiments, using biological replicates. No statistical methods were used to predetermine sample size for in vitro experiments. Sample sizes were selected empirically from previous experimental experience, and/or from sizes generally employed in the field. For in vivo transplantation experiments, the minimal number of mice in each group was calculated through use of the 'cpower' function in the R/Hmisc package. The precise number of sample used in indicated in manuscript. The precise number of sample used in indicated in manuscript.

Data exclusions No data were excluded from the analyses.For all FACS analysis, only single cells were included in further analysis. Duplets determined by FSC$\mathrm{H} /$ FCS-A as well as SSC-H/SSA-A were excluded. This was done to avoid false positive events due to doublets (Kudernatsch RF et al. Cytometry A 2013, PMID 23281028).

Replication The experimental findings were reliably reproduced using biological replicates and multiple cells indicated in manuscript.

Randomization Samples and animals were randomly divided into experimental groups.

Blinding Mice were randomly allocated to groups by investigators who did not participate in any subsequent analysis. The performers of the mouse experiments (injections, drug treatments) were blinded to the randomization process.

\section{Reporting for specific materials, systems and methods}

We require information from authors about some types of materials, experimental systems and methods used in many studies. Here, indicate whether each material, system or method listed is relevant to your study. If you are not sure if a list item applies to your research, read the appropriate section before selecting a response.

\begin{tabular}{l|l}
\multicolumn{1}{l}{ Materials \& experimental sys } \\
\hline $\mathrm{n} / \mathrm{a}$ & Involved in the study \\
\hline & $\boldsymbol{x}$. Antibodies \\
\hline & $\boldsymbol{x}$ Eukaryotic cell lines \\
\hline $\boldsymbol{x}$ & $\square$ Palaeontology \\
$\square$ & $\boldsymbol{x}$ Animals and other organisms \\
$\square$ & $\boldsymbol{x}$ Human research participants \\
$\boldsymbol{x}$ & $\square$ Clinical data
\end{tabular}

\begin{tabular}{l|l}
\multicolumn{2}{l}{ Methods } \\
\hline n/a & Involved in the study \\
$\square$ & $\boldsymbol{x}$ ChIP-seq \\
$\square$ & $\boldsymbol{x}$ Flow cytometry \\
\hline $\boldsymbol{x}$ & $\square$ MRI-based neuroimaging
\end{tabular}

\section{Antibodies}

Antibodies used

\author{
Antibodies for Western blot \\ Polyclonal anti-STAT1-pY701 Cell Signaling Technology Cat\# 9172 (1:1000) \\ Polyclonal anti-STAT1 Cell Signaling Technology Cat\# 5605 (1:1000) \\ Polyclonal anti-STAT5-pY694 Cell Signaling Technology Cat\# 9351 (1:1000) \\ Polyclonal anti-STAT5 Cell Signaling Technology Cat\# 9363 (1:1000) \\ Polyclonal anti-IFITM3 Cell Signaling Technology Cat\# 9037 (1:1000) \\ Polyclonal anti-AKT-pS473 Cell Signaling Technology Cat\# 9271 (1:750) \\ Polyclonal anti-AKT Cell Signaling Technology Cat\# 9272 (1:1000) \\ Polyclonal anti-ERK-pT202/Y204 Cell Signaling Technology Cat\# 4370 (1:1000) \\ Polyclonal anti-ERK Cell Signaling Technology Cat\# 9102 (1:1000) \\ Polyclonal (D8E8G) anti-IFITM3 Cell Signaling Technology Cat\# 59212 (1:1000) \\ Polyclonal (D84C12) anti-c-Myc Cell Signaling Technology Cat\# 5605 (1:1000) \\ Polyclonal anti-c-Abl-pY412 Cell Signaling Technology Cat\# 2865 (1:1000) \\ Polyclonal anti-c-Abl Santa Cruz Biotechnology Cat\# sc-131 (1:1000) \\ Polyclonal anti-Src-pY416 Cell Signaling Technology Cat\# 6943 (1:1000) \\ Polyclonal anti-Lyn Cell Signaling Technology Cat\# 2796 (1:1000)
}


Polyclonal anti-Syk-pY352 Cell Signaling Technology Cat\# 2717 (1:1000)

Polyclonal anti-Syk Cell Signaling Technology Cat\# 2712 (1:1000)

Polyclonal (C-2) anti-Bcl-2 Santa Cruz Biotechnology Cat\# sc-7382 (1:1000)

Polyclonal anti-p21 (C-19) Santa Cruz Biotechnology Cat\# sc-397 (1:1000)

Polyclonal anti-CD19-pY531 Cell Signaling Technology Cat\# 3571 (1:1000)

Monoclonal (D4V4B) anti-CD19 Cell Signaling Technology Cat\# 90176 (1:1000)

Monoclonal (1C12) anti-p53 Cell Signaling Technology Cat\# 2524 (1:1000)

Polyclonal anti-p19/Arf ABCAM Cat\# ab80 (1:1000)

Monoclonal (4G10) anti-Phosphotyrosine EMD Millipore Cat\# 05-321 (1:1000)

Polyclonal anti-HA tag ABCAM Cat\# ab9110 (1:1000)

Polyclonal (D6N9Y) anti-IKZF1 Cell Signaling Technology Cat\# 14859 (1:1000)

Polyclonal anti-PIK3CD; p1108 ABCAM Cat\# ab109006 (1:1000)

Monoclonal (M2) anti-FLAG Sigma-Aldrich Cat\# F1804 (1:1000)

Polyclonal anti-elF4E Santa Cruz Biotechnology Cat\# sc-13963 (1:1000)

Polyclonal anti-LYN-pY397 Cell Signaling Technology Cat\# 70926 (1:1000)

Monoclonal (108D2) anti-p70 S6K-pT389 Cell Signaling Technology Cat\# 9234 (1:1000)

Polyclonal anti-p70 S6K-pT421/S424 Cell Signaling Technology Cat\# 9204 (1:1000)

Monoclonal (49D7) anti-p70 S6 Kinase Cell Signaling Technology Cat\# 2708 (1:1000)

Monoclonal (D30A3) anti-RICTOR-pT1135 Cell Signaling Technology Cat\# 3806 (1:1000)

Monoclonal (53A2) anti-RICTOR Cell Signaling Technology Cat\# 2114 (1:1000)

Polyclonal anti-CXCR4-pS339 Cell Signaling Technology Cat\# 59028 (1:1000)

Polyclonal anti-CXCR4 Novus Biologicals Cat\# 74396 (1:1000)

Polyclonal anti-FAK-pY397 Cell Signaling Technology Cat\# 3283 (1:1000)

Polyclonal anti-FAK-pY575/577 Cell Signaling Technology Cat\# 3281 (1:1000)

Monoclonal (D2R2E) anti-FAK Cell Signaling Technology Cat\# 13009 (1:1000)

Polyclonal anti-PAK1-pS199/204 Cell Signaling Technology Cat\# 2605 (1:1000)

Polyclonal anti-PAK1 Cell Signaling Technology Cat\# 2602 (1:1000)

Polyclonal anti-ITGB1-pY783 ABCAM Cat\# ab62337 (1:1000)

Polyclonal anti-ITGB1-pT788/789 Thermo Fisher Scientific Cat\# 44-872G (1:1000)

Polyclonal anti-ITGB1 Cell Signaling Technology Cat\# 4706 (1:1000)

Monoclonal (C4) anti- $\beta$-actin Santa Cruz Biotechnology Cat\# sc-47778 (1:5000)

Antibodies for FACS

Monoclonal (HIB19) anti-human CD19 Biolegend Cat\# 302254 (1:50)

Monoclonal (M2) anti-FLAG Columbia Biosciences Cat\# M2 (1:200)

Polyclonal anti-HA tag Columbia Biosciences Cat\# D5-1718 (1:200)

Monoclonal (OKT4) anti-human CD4 Biolegend Cat\# 317418 (1:50)

Monoclonal (UCHT1) anti-human CD3 Biolegend Cat\# 555335 (1:50)

Monoclonal (G20-127) anti-human IgM BD Biosciences Cat\# 561285 (1:50)

Monoclonal (6D5) anti-mouse CD19 Biolegend Cat\# 115508 (1:200)

Monoclonal (RA3-6B2) anti-B220 (CD45R) Biolegend Cat\# 103224 (1:200)

Monoclonal (HM79-12) anti-CD79b Biolegend Cat\# 132804 (1:200)

Monoclonal (RB6-8C5) anti-mouse Gr-1 (Ly-6GC) Biolegend Cat\# 108422 (1:200)

Monoclonal (PK136) anti-mouse NK-1.1 Biolegend Cat\# 108730 (1:200)

Monoclonal (2B8) anti-CD117 (c-kit) Biolegend Cat\# 105808 (1:200)

Monoclonal (D7) anti-Sca-1 (Ly-6A/E) Biolegend Cat\# 108102 (1:200)

Monoclonal (S11) anti-mouse CD43 Biolegend Cat\# 143208 (1:200)

Monoclonal (6C3) anti-mouse BP-1 (Ly-51) Biolegend Cat\# 108308 (1:200)

Monoclonal (M1/69) anti-mouse CD24 Biolegend Cat\# 101822 (1:200)

Monoclonal (R6-60.2) anti-mouse IgM BD Biosciences Cat\# 553408 (1:200)

Monoclonal (11-26c.2a) anti-mouse IgD Biolegend Cat\# 405716 (1:200)

Monoclonal (B3B4) anti-mouse CD23 BD Biosciences Cat\# 561773 (1:200)

Monoclonal (7E9) anti-mouse CD21/CD35 Biolegend Cat\# 123412 (1:200)

Monoclonal (M1/70) anti-mouse Mac-1 (CD11b) Biolegend Cat\# 101206 (1:200)

Monoclonal (53-7.3) anti-mouse CD5 Biolegend Cat\# 100612 (1:200)

Monoclonal (17A2) anti-mouse CD3 Biolegend Cat\# 100204 (1:200)

Monoclonal (RM4-5) anti-mouse CD4 BD Biosciences Cat\# 553051 (1:200)

Monoclonal (Jo2) anti-mouse CD95 BD Biosciences Cat\# 562499 (1:200)

Monoclonal (GL7) anti-mouse GL7 Affymetrix eBioscience Cat\# 13-5902-82 (1:200)

Monoclonal (L276F12) anti-mouse CXCR4 Biolegend Cat\# 146508 (1:200)

Monoclonal (GL-1) anti-mouse CD86 Biolegend Cat\# 105016 (1:200)

Monoclonal (A7R34) anti-mouse CD127 (IL-7R) eBioscience Cat\# 135012 (1:200)

Monoclonal (OX-7) anti-mouse Thy1 (CD90) BD Biosciences Cat\# 551401 (1:200)

Monoclonal (RM2-5) anti-mouse CD2 Biolegend Cat\# 100108 (1:200) 
Monoclonal (HM48-1) anti-mouse CD48 Biolegend Cat\# 103415 (1:200)

Monoclonal (IM7) anti-mouse CD44 Biolegend Cat\# 103012 (1:200)

Monoclonal (MEC13.3) anti-mouse Pecam1 Biolegend Cat\# 102508 (1:200)

Monoclonal (TY/23) anti-mouse CD73 (5NTD) BD Biosciences Cat\# 550741 (1:200)

Monoclonal (37.51) anti-mouse CD28 BD Biosciences Cat\# 553297 (1:200)

Monoclonal (4G2) anti-mouse CD319 Biolegend Cat\# 152004 (1:200)

Monoclonal (OX-97) anti-mouse CD22 Biolegend Cat\# 126112 (1:200)

Polyclonal anti-GLUT3 Alomone labs Cat\# AGT-023 (1:200)

Monoclonal (93) anti-mouse CD16/32 Biolegend Cat\# 101314 (1:200)

Monoclonal (2G10) anti-CIP2A Novus Biologicals Cat\#59722APC (1:200)

Monoclonal (SA15-21) anti-mouse TLR4 (CD284) Biolegend Cat\# 145404 (1:200)

Polyclonal anti-KV1.3 (KCNA3) Alomone labs Cat\# APC-101 (1:200)

Polyclonal anti-Insulin R/CD220 R\&D Systems Cat\# FAB1544A (1:200)

Monoclonal (P84) anti-mouse CD172a (SIRP $\alpha$ ) Biolegend Cat\# 144014 (1:200)

Polyclonal anti-ABCG1 Invitrogen Cat\# PA5-72939 (1:200)

monoclonal (EPR13130) anti-TMEM173 ABCAM Cat\# ab208874 (1:200)

Polyclonal anti-ORP8 (OSBPL8) Biorbyt Cat\# orb496769 (1:200)

Polyclonal anti-OSBPL5 Biorbyt Cat\# orb189989 (1:200)

Polyclonal anti-Squalene Epoxidase Biorbyt Cat\# orb7410 (1:200)

Monoclonal (22H9) anti-mouse TSLPR (TSLP-R) Biolegend Cat\# 151806 (1:200)

Monoclonal (MAb-CC1) anti-mouse Ceacam1 (CD66a) Biolegend Cat\# 134524 (1:200)

Monoclonal (AA4.1) anti-mouse CD93 Biolegend Cat\# 136504 (1:200)

Monoclonal (L276F12) anti-mouse CD184 (CXCR4) Biolegend Cat\# 146508 (1:200)

Monoclonal (A7R34) anti-mouse CD127 (IL-7R $\alpha$ ) Biolegend Cat\# 135012 (1:200)

Monoclonal (PC61) anti-CD25 Biolegend Cat\# 102008 (1:200)

Monoclonal (K10.6) anti-mouse CD72 a, b and d BD Biosciences Cat\# 550966 (1:200)

Monoclonal (mCD44.7) anti-mouse CD84 Biolegend Cat\# 122806 (1:200)

Brilliant Violet 421 ${ }^{\mathrm{TM}}$ Streptavidin Biolegend Cat\# 405225 (1:200)

Monoclonal (5H10-27(MFR5)) anti-mouse CD49e (Integrin a5) Biolegend Cat\# 103805 (1:200)

Monoclonal (GoH3) anti-mouse CD49f (Integrin a6) Biolegend Cat\# 313612 (1:200)

Monoclonal (MEL-14) anti-mouse CD62L (L-selectin) Biolegend Cat\# 104408 (1:200)

Monoclonal (HMß1-1) anti-mouse CD29 (Integrin B1) Biolegend Cat\# 102216 (1:200)

Monoclonal (37.51) anti-mouse CD28 BD Biosciences Cat\# 553297 (1:200)

Monoclonal (SA275A11) anti-mouse CD20 Biolegend Cat\# 150410 (1:200)

Anti Ct-B (Cholera toxin subunit B) antibody Thermo Fisher Scientific Cat\# V34405 (1:200)

Antibodies for PLA

Polyclonal anti-human IgM OriGene Cat\# AP10433PU-N (1:150)

Polyclonal anti-human LAMP1 R \& D Systems Cat\# IC7985G (1:150)

Polyclonal (D8E8G) anti-IFITM3 Cell Signaling Technology Cat\# 59212 (1:150)

Monoclonal (CB3-1) anti-CD79B Thermo Fisher Scientific Cat\# 14-0793-82 (1:150)

Antibodies for immunofluorescence, Immunization

Biotinylated Peanut Agglutinin (PNA) Vector Laboratories Cat\# B-1075 (1:200)

Alexa Fluor ${ }^{\circledR} 647$ Streptavidin Biolegend Cat\# 405237 (1:200)

Polyclonal (RA3-6B2) anti-B220 BD Biosciences Cat\# 553089 (1:200)

Monoclonal (17A2) anti-mouse CD3 Biolegend Cat\# 100214 (1:200)

Antibody for lipid strip

Polyclonal anti-GST tag Thermo Fisher Scientific Cat\# CAB4169 (1:1000)

Monoclonal (D5A7) anti-Biotin Cell Signaling Technology Cat\# 5597 (1:1000)

Antibodies for cell stimulation.

(For the Measurement of intracellular calcium mobilization, $10 \mu \mathrm{g} \mathrm{ml}-1$ of antibodies were added to $1 \times 106$ viable cells. For the In vitro crosslinking, $2.5 \mu \mathrm{g} \mathrm{ml}-1$ of antibodies were added to $1 \times 106$ viable cells.)

Polyclonal anti-HA tag ABCAM Cat\# ab9110

Monoclonal (1D3) purified NA/LE anti-mouse CD19 BD Biosciences Cat\# 553782

Polyclonal anti-mouse IgM Southern Biotech Cat\# 1020-01

Polyclonal anti-human IFITM3 Bioss Cat\# bs-12265R

Polyclonal $F\left(a b^{\prime}\right) 2$ anti-human $\mu$ chain Jackson Immunoresearch Cat\# 109-006-129

Monoclonal (OKT3) purified LE/AF anti-human CD3 Biolegend Cat\# 317304 
See manufactures websites for validation statements (www.abcam.com; www.cellsignal.com; https://www.bdbiosciences.com/ en-us; h ttps://www.biolegend.com; https://www.thermofisher.com; https://www.scbt.com; https://www.novusbio.com/; https://www.emdmillipore.com; https://www.sigmaaldrich.com; https://columbiabiosciences.com; https://www.alomone.com; https://www.rndsystems.com; https://www.biorbyt.com; https://www.origene.com

\section{Eukaryotic cell lines}

Policy information about cell lines

Cell line source(s)

BV-173 Ph+ ALL carrying BCR-ABL1, MAP3K14 and IKZF1 lesions from DSMZ.

JEKO1 Mantle cell lymphoma carrying IGH-CCND1, ITK, MAP2K1 and TP53 lesions from ATCC. Jurkat T-ALL carrying PTEN, LCK and CD4 lesions from ATCC.

Detail information for patient-derived samples used are listed in Supplementary table 7 and 8.

HEK293FT cells were used for generation of retroviruses and lentiviruses (see Methods).

Authentication

Patient-derived cells or cell lines used in this study (see Supplementary tables 7 and 8) and HEK293FT cells were validated by STR DNA profiling analysis.

\section{Mycoplasma contamination}

The cell line tested negative for mycoplasma contamination by PCR analysis.

Commonly misidentified lines (See ICLAC register)

\section{Animals and other organisms}

Policy information about studies involving animals; ARRIVE guidelines recommended for reporting animal research

Laboratory animals

Wild animals

Field-collected samples

Ethics oversight
All mouse models used in the study are listed in Supplementary Table S9. NOD.Cg-Prkdcscid II rgtm1Wjl/SzJ (NSG) were purchased from Jackson Laboratory. Ifitm3tm1Masu from Dr. Michael S. Diamond were backcrossed to C57BL/6J background for more than 8 generations. B6.C(Cg)-Cd79atm1(cre)Reth/EhobJ (Mb1-Cre) were purchased from Jackson Laboratory. B6.129S2Ighmtm1Cgn/J (muMT mice) were purchased from Jackson Laboratory. C;129S4-Ptentm1Hwu/J (Ptenfl/fl) were purchased from Jackson Laboratory. To generate a model for pre-leukemic B cell precursors expressing BCR-ABL1, LSL-BCr+/ BCR-ABL1 mice were crossed with Mb1-Cre strain (Mb1-Cre x LSL-Bcr+/ BCR-ABL1) for excision of a stop-cassette in early pre-B cells. For in vivo oncogenic priming assay with Mb1-Cre x LSL-BCr+/ BCR-ABL1 B-cell precursors, 8- to 10-week-old female NSG mice were randomly allocated before injection. For animals bred in house, littermates of the same sex were randomized to experimental groups. For in vivo leukemia initiation assay 8- to 10-week-old female NSG mice were randomly allocated before injection. All mouse experiments were subject to institutional approval by the Beckman Research Institute of City of Hope Animal Care and Use Committee.

Temperatures of $18-23^{\circ} \mathrm{C}$ with $40-60 \%$ humidity were maintained with 14 -hour light/10-hour dark cycle. Following score was considered as end-point. 1. Failure to eat food / drink water for 24 hours. 2. Failure to make normal postural adjustments /

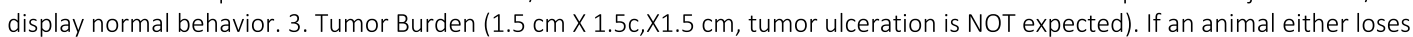
$25 \%$ of the initial body weight (or reaches $16 \mathrm{~g}$ of body weight, regardless of the initial weight) or if we observe a weight loss of $15 \%$ on two sequential weight measurements, we euthanized the mouse immediately.

This study did not involve wild animals.

This study did not involve samples collected from the fields.

All mouse breeding experiments were subject to institutional approval by by the Beckman Research Institute Animal Care and Use Committee. Patient samples (Table S9) were obtained in compliance with the internal review board of the Beckman Research Institute of City of Hope.

Note that full information on the approval of the study protocol must also be provided in the manuscript.

\section{Human research participants}

Policy information about studies involving human research participants

Population characteristics

GSE expression profiles of diagnostic samples from gene expression microarray data from three large cohorts of patients with pre-B ALL were studied from GSE5314 (the Eastern Cooperative Oncology Group (ECOG) Clinical Trial E2993, Juric et al., 2007), GSE11877 (the Children's Oncology Group (COG) Clinical Trial P9906, Harvey et al., 2010; Kang et al., 2010), St. Jude Research Hospital pediatric ALL (Ross et al., 2003, http://www.stjuderesearch.org/site/data/ALL3/). Patients had MRD tested by flow cytometry with two combinations (CD20/CD10/CD45 or CD9/CD19/CD34/CD45), and were defined as MRD positive or MRD negative at the end of induction therapy (day 29) using a threshold of $0.01 \%$ as previously described (Borowitz, M. J. et al. 2008). Then, RNA was purified from 207 pretreatment diagnostic samples with more than $80 \%$ blasts ( 131 bone marrow, 76 peripheral blood) and subjected to microarrays. Log-rank test was used to assess statistical significance. Patient-outcome data with mantle cell lymphoma were studied (https://Impp.nih.gov/MCL/). Patient-outcome data for AML were studied from TCGA Acute Myeloid Leukemia Project (http://www.cbioportal.org/study/summary?id=laml_tcga_pub\#clinical; Cancer Genome Atlas Research Network, 2013). 
Recruitment

Ethics oversight
We are not aware of any potential self-selection bias or other biases present.

Human leukemia samples were sourced ethically and their use was in compliance with the internal review boards of the Beckman Research Institute of City of Hope.

Note that full information on the approval of the study protocol must also be provided in the manuscript.

\section{ChIP-seq}

\section{Data deposition}

$\boldsymbol{x}$ Confirm that both raw and final processed data have been deposited in a public database such as GEO.

Confirm that you have deposited or provided access to graph files (e.g. BED files) for the called peaks.

Data access links

May remain private before publication.

Files in database submission

Genome browser session (e.g. $\underline{\text { UCSC)}}$

\section{Methodology}

Replicates

\section{Sequencing depth}

Antibodies

Peak calling parameters

Data quality

Software
Genome wide mapping of IKZF1 binding (ChIP-Seq) in human patient-derived B-ALL xenograft cells (Schjerven H. et al., 2017, GSE58825); Genome binding/occupancy profiling of activation of Cre in Ikzf1exon5fl/fl stromal adherent pre-B cells (Hu Y. et al., 2016, GSE86897)

Genome wide mapping of IKZF1 binding (ChIP-Seq) in human patient-derived B-ALL xenograft cells (Schjerven H. et al., 2017, GSE58825); Genome binding/occupancy profiling of activation of Cre in lkzf1exon5fl/fl stromal adherent pre-B cells (Hu Y. et al., 2016, GSE86897)

Genome wide mapping of IKZF1 binding (ChIP-Seq) in human patient-derived B-ALL xenograft cells (Schjerven H. et al., 2017, GSE58825); Genome binding/occupancy profiling of activation of Cre in Ikzf1exon5fl/fl stromal adherent pre-B cells (Hu Y. et al., 2016, GSE86897)
Genome wide mapping of IKZF1 binding (ChIP-Seq) in human patient-derived B-ALL xenograft cells (Schjerven H. et al., 2017, GSE58825); Genome binding/occupancy profiling of activation of Cre in Ikzf1exon5fl/fl stromal adherent pre-B cells (Hu Y. et al., 2016, GSE86897)

N/A

antibodies for RNAPII, H3K4me3 and IKZF1

The ChIP-seq peaks were called by the MACS peak caller by comparing read density in the ChIP experiment relative to the input chromatin control reads, and are shown as bars under each wiggle track.

N/A

Integrative Genomics Viewer (IGV) was used to visualize ChIP-seq tracks downloaded from ENCODE.

\section{Flow Cytometry}

\section{Plots}

Confirm that:

$\boldsymbol{x}$ The axis labels state the marker and fluorochrome used (e.g. CD4-FITC).

$\boldsymbol{x}$ The axis scales are clearly visible. Include numbers along axes only for bottom left plot of group (a 'group' is an analysis of identical markers).

$\boldsymbol{x}$ All plots are contour plots with outliers or pseudocolor plots.

$\boldsymbol{x}$ A numerical value for number of cells or percentage (with statistics) is provided.

\section{Methodology}

\section{Sample preparation}

Instrument

Software

Cell population abundance
PBS washed cells were blocked with Fc blocker for 10 min on ice and then stained with the appropriate antibodies or isotype control for $25 \mathrm{~min}$ on ice. Cells were then washed and resuspended in chilled PBS containing $0.75 \mu \mathrm{g} / \mathrm{ml}$ of DAPI to exclude dead cells.

Acquisition was performed by LSRFortessa flow cytometer (BD Biosciences). The fluorescence based cell sorting was performed by FACSAria II (BD Biosciences).

FACS data were analyzed with FlowJo software (FlowJo, LLC).

At least 20,000 cells per sample were recorded. Purity was determined by comparison with negative control. 
$\boldsymbol{x}$ Tick this box to confirm that a figure exemplifying the gating strategy is provided in the Supplementary Information. 